\title{
DESINGULARIZATION OF CLIFFORD TORUS AND NONRADIAL SOLUTIONS TO THE YAMABE PROBLEM WITH MAXIMAL RANK
}

\author{
MARIA MEDINA, MONICA MUSSO, AND JUNCHENG WEI
}

\begin{abstract}
Through desingularization of Clifford torus, we prove the existence of a sequence of nondegenerate (in the sense of Duyckaerts-Kenig-Merle ([7])) nodal nonradial solutions to the critical Yamabe problem

$$
-\Delta u=\frac{n(n-2)}{4}|u|^{\frac{4}{n-2}} u, \quad u \in \mathcal{D}^{1,2}\left(\mathbb{R}^{n}\right) .
$$

The case $n=4$ is the first example in the literature of a solution with maximal rank $\mathcal{N}=$ $2 n+1+\frac{n(n-1)}{2}$.
\end{abstract}

\section{Introduction}

Consider the problem

$$
-\Delta u=\gamma|u|^{p-1} u \text { in } \mathbb{R}^{n}, \quad \gamma:=\frac{n(n-2)}{4}, \quad u \in \mathcal{D}^{1,2}\left(\mathbb{R}^{n}\right),
$$

where $n \geqslant 4, p=\frac{n+2}{n-2}$ and $\mathcal{D}^{1,2}\left(\mathbb{R}^{n}\right)$ is the completion of $C_{0}^{\infty}\left(\mathbb{R}^{n}\right)$ with the norm $\|\nabla u\|_{L^{2}\left(\mathbb{R}^{n}\right)}$.

When $u>0$, problem (0.0.1) arises in the classical Yamabe problem or extremal equation for Sobolev inequality. For positive or sign-changing $u$ Problem (0.0.1) corresponds to the steady state of the energy-critical focusing nonlinear wave equation

$$
\partial_{t}^{2} u-\Delta u-|u|^{\frac{4}{n-2}} u=0,(t, x) \in \mathbb{R} \times \mathbb{R}^{n} .
$$

These are classical problems that have attracted the attention of many researchers $([\overline{8}, 19,15,16$, 18]). The study of (0.0.2) naturally relies on the complete classification of the set of non-zero finite energy solutions to Problem (0.0.1), which is defined by

$$
\Sigma:=\left\{Q \in \mathcal{D}^{1,2}\left(\mathbb{R}^{n}\right) \backslash\{0\}:-\Delta Q=\frac{n(n-2)}{4}|Q|^{\frac{4}{n-2}} Q\right\} .
$$

By the classical work of Caffarelli-Gidas-Spruck [2] all positive solutions to (0.0.1) are given by

$$
U(y)=\left(\frac{2}{1+|y|^{2}}\right)^{\frac{n-2}{2}},
$$

The first author is supported by the grant FONDECYT Postdoctorado, No. 3160077, CONICYT (Chile) and grant MTM2013-40846-P, MINECO (Spain). The second author is supported by FONDECYT Grant 1160135 and Millennium Nucleus Center for Analysis of PDE, NC130017. The third author is supported by NSERC of Canada. 
and all its translations and dilations

$$
U_{\alpha, \bar{y}}:=\alpha^{-\frac{n-2}{2}} U\left(\frac{y-\bar{y}}{\alpha}\right), \quad \alpha>0, \bar{y} \in \mathbb{R}^{n} .
$$

For sign-changing solutions much less is known. A direct application of Pohozaev's identity gives that all sign-changing solutions to Problem (0.0.1) are nonradial. The existence of elements of $\Sigma$ that are nonradial, sign-changing, and with arbitrary large energy was first proved by Ding [6] using Ljusternik-Schnirelman category theory. However no other qualitative properties are known for Ding's solutions. Recently more explicit constructions of sign-changing solutions to Problem (0.0.1) have been obtained by del Pino-Musso-Pacard-Pistoia [4, 5]. In [20, the second and the third authors established the rigidity of the solutions constructed in [4] by showing that they are nondegenerate in the sense of Duyckaerts-Kenig-Merle ([7], see definitions below).

The purpose of this work is to give a positive answer to an open question formulated in the work of M. Musso and J. Wei $([20])$ : whether there exists a solution that, apart from nondegenerate, is maximal. To properly explain this framework, let us denote by

$$
\Sigma:=\left\{Q \in \mathcal{D}^{1,2}\left(\mathbb{R}^{n}\right) \backslash\{0\}:-\Delta Q=\gamma|Q|^{p-1} Q\right\}
$$

the set of nontrivial finite energy solutions of (0.0.1). It can be seen that the equation in (0.0.1) is invariant under four transformations: translation, dilation, orthogonal transformation and Kelvin transform. More precisely, if $Q \in \Sigma$, then:

(i) $Q(y+a) \in \Sigma$ for every $a \in \mathbb{R}^{n}$;

(ii) $\lambda^{\frac{n-2}{2}} Q(\lambda y) \in \Sigma$ for every $\lambda>0$;

(iii) $Q(P y) \in \Sigma$ for every $P \in \mathcal{O}_{n}$, where $\mathcal{O}_{n}$ denotes the classical orthogonal group;

(iv) $|y|^{2-n} Q\left(|y|^{-2} y\right) \in \Sigma$.

Denote by $\mathcal{M}$ the group of isometries of $\mathcal{D}^{1,2}\left(\mathbb{R}^{n}\right)$ generated by these transformations. Then, $\mathcal{M}$ derives a family of transformations in a neighborhood of the identity (see [7, Lemma 3.8]) of dimension

$$
\mathcal{N}:=2 n+1+\frac{n(n-1)}{2} .
$$

In particular, $\mathcal{M}$ generates the vector space

$$
\tilde{\mathcal{J}}_{Q}=\operatorname{span}\left\{\begin{array}{c}
(2-n) y_{\alpha} Q+|y|^{2} \partial_{y_{\alpha}} Q-2 y_{\alpha} y \cdot \nabla Q, \quad \partial_{y_{\alpha}} Q, \quad 1 \leqslant \alpha \leqslant n, \\
\left(y_{\alpha} \partial_{y_{\beta}}-y_{\beta} \partial_{y_{\alpha}}\right) Q, \quad 1 \leqslant \alpha<\beta \leqslant n, \quad \frac{n-2}{2} Q+y \cdot Q
\end{array}\right\} .
$$

Consider the associated linearized operator around $Q \in \Sigma$, i.e.,

$$
L_{Q}:=-\Delta-\gamma p|Q|^{p-2} Q,
$$

and its kernel

$$
\mathcal{J}_{Q}:=\left\{f \in \mathcal{D}^{1,2}\left(\mathbb{R}^{n}\right): L_{Q} f=0\right\} .
$$

Clearly $\tilde{\mathcal{J}}_{Q} \subseteq \mathcal{J}_{Q}$ and, following the work of T. Duyckaerts, C. Kenig and F. Merle ([7]), we can define the notion of nondegeneracy.

Definition 0.0.1. $Q \in \Sigma$ is said to be nondegenerate if $\mathcal{J}_{Q}=\tilde{\mathfrak{J}}_{Q}$. 
Let $Q$ be nondegenerate. Its rank is defined as the dimension of $\tilde{\mathcal{J}}_{Q}$, which is at most $\mathcal{N}$. Actually, the positive solutions $Q=W$ can be proved to be nondegenerate as a consequence of the radial symmetry, and $\tilde{\mathcal{J}}_{W}$, which is

$$
\tilde{\mathcal{J}}_{W}=\left\{\frac{n-2}{2} W+y \cdot \nabla W, \quad \partial_{y_{\alpha}} W, \quad 1 \leqslant \alpha \leqslant n\right\},
$$

has rank $n+1$ ([21]). In this case, the rank is strictly less than $\mathcal{N}$.

In [20], the authors give the first example of nodal nonradial sign-changing solution satisfying the nondegeneracy condition. Indeed, they consider the solution $u_{k}$ of (0.0.1) built in [4, Theorem 1] given by

$$
u_{k}(y)=U(y)-\sum_{j=1}^{k} \mu_{k}^{-\frac{n-2}{2}} U\left(\mu_{k}^{-1}\left(y-\xi_{j}\right)\right)+o(1),
$$

where

$$
\mu_{k}:=\frac{c_{n}}{k^{2}}, \quad \xi_{j}:=\left(e^{\frac{2 j \pi i}{k}}, 0, \ldots\right), \quad U(y):=\left(\frac{2}{1+|y|^{2}}\right)^{\frac{n-2}{2}},
$$

and they prove that $\tilde{\mathcal{J}}_{u_{k}}=\mathcal{J}_{u_{k}}$, where the dimension of these vector spaces is $3 n$, i.e. the rank is $3 n$. Also in this case, the rank is strictly less than $\mathcal{N}$.

The purpose of this work is to provide the first example in the literature of a nondegenerate solution $u$ to (0.0.1) which has the maximal rank $\mathcal{N}$.

Definition 0.0.2. A nondegenerate solution $Q \in \Sigma$ is said to be maximal if

$$
\operatorname{dim}\left(\tilde{\mathcal{J}}_{Q}\right)=\operatorname{dim}\left(\mathcal{J}_{Q}\right)=\mathcal{N},
$$

where $\mathcal{N}$ was defined in $(0.0 .6$.

Thus, our main result can be formulated as follows.

Theorem 0.0.3. Let $n \geqslant 4$. Then, there exists a sequence of nodal solutions to (0.0.1), with arbitrarily large energy, which are nondegenerate according to Definition 0.0.1. If $n=4$ these solutions are maximal in the sense of Definition 0.0.2.

To prove this result, we will build a solution in the following way: let $k$ and $h$ be two large positive integers (not necessarily equal), and

$$
\mu:=\frac{\delta^{\frac{2}{n-2}}}{k^{2}}, \quad \lambda:=\frac{\varepsilon^{\frac{2}{n-2}}}{h^{2}}
$$

where $\delta$ and $\varepsilon$ are positive parameters so that

$$
c_{1}<\delta<c_{1}^{-1}, \quad c_{2}<\varepsilon<c_{2}^{-1},
$$

for some constants $c_{1}, c_{2}>0$ which are independent of $k$ and $h$ as they tend to infinity. Consider now the points

$$
\begin{aligned}
\xi_{j} & :=\sqrt{1-\mu^{2}}\left(e^{\frac{2 \pi i(j-1)}{k}}, 0, \ldots, 0\right) \in \mathbb{R}^{2} \times \mathbb{R}^{n-2}, j=1, \ldots, k, \\
\eta_{l} & :=\sqrt{1-\lambda^{2}}\left(0,0, e^{\frac{2 \pi i(l-1)}{h}}, 0, \ldots, 0\right) \in \mathbb{R}^{2} \times \mathbb{R}^{2} \times \mathbb{R}^{n-4}, l=1, \ldots, h,
\end{aligned}
$$

which satisfy

$$
\left|\xi_{j}\right|^{2}+\mu^{2}=1, \quad\left|\eta_{l}\right|^{2}+\lambda^{2}=1
$$


Consider

$$
u(y)=U(y)-\sum_{j=1}^{k} U_{\mu, \xi_{j}}(y)-\sum_{l=1}^{h} U_{\lambda, \eta_{l}}(y)+\phi(y)
$$

where $U$ is defined in (0.0.4),

$$
U_{\mu, \xi_{j}}(y):=\mu^{-\frac{n-2}{2}} U\left(\frac{y-\xi_{j}}{\mu}\right), \quad U_{\lambda, \eta_{l}}(y):=\lambda^{-\frac{n-2}{2}} U\left(\frac{y-\eta_{l}}{\lambda}\right),
$$

and $\phi$ is a small function when compared with the other terms (for the sake of simplicity we do not make explicit the dependence of $u$ in $k$ and $h$ ).

Notice that functions $U, U_{\mu, \xi_{j}}$ and $U_{\lambda, \eta_{l}}$ are invariant under rotation of angle $\frac{2 \pi}{k}$ in the $\left(y_{1}, y_{2}\right)$ plane and of angle $\frac{2 \pi}{h}$ in the $\left(y_{3}, y_{4}\right)$ angle. Furthermore, they are even in the $y_{\alpha}$-coordinates, for $\alpha=2,4,5, \ldots, n$ and invariant under Kelvin's transform (due to (0.0.9)). Assume that $\phi$ also satisfies these properties (we will prove this in Part 1).

Consider the following set of functions:

$$
\begin{gathered}
z_{0}(y):=\frac{n-2}{2} u(y)+\nabla u(y) \cdot y, \\
z_{\alpha}(y):=\frac{\partial}{\partial y_{\alpha}} u(y), \quad \alpha=1, \ldots, n, \\
z_{n+1}(y):=-y_{2} \frac{\partial}{\partial y_{1}} u(y)+y_{1} \frac{\partial}{\partial y_{2}} u(y), \\
z_{n+2}(y):=-y_{4} \frac{\partial}{\partial y_{3}} u(y)+y_{3} \frac{\partial}{\partial y_{4}} u(y), \\
z_{n+\alpha+2}(y):=-2 y_{\alpha} z_{0}(y)+|y|^{2} z_{\alpha}(y), \quad \alpha=1,2,3,4, \\
z_{n+\alpha+4}(y):=-y_{\alpha} z_{1}(y)+y_{1} z_{\alpha}(y), \quad \alpha=3, \ldots, n, \\
z_{2 n+\alpha+2}(y):=-y_{\alpha} z_{2}(y)+y_{2} z_{\alpha}(y), \quad \alpha=3, \ldots, n,
\end{gathered}
$$

and

$$
\begin{array}{ll}
z_{3 n+\alpha-2}(y):=-y_{\alpha} z_{3}(y)+y_{3} z_{\alpha}(y), & \alpha=5, \ldots, n, \\
z_{4 n+\alpha-6}(y):=-y_{\alpha} z_{4}(y)+y_{4} z_{\alpha}(y), & \alpha=5, \ldots, n .
\end{array}
$$

Functions (0.0.12) and (0.0.13) are related to the invariance of problem (0.0.1) under dilations and translations respectively, and (0.0.14) to the rotation in the $\left(y_{1}, y_{2}\right)$ and $\left(y_{3}, y_{4}\right)$ planes. Likewise, (0.0.15) arises from the invariance under Kelvin transform, and (0.0.16), (0.0.17) from the rotation in the planes $\left(y_{1}, y_{\alpha}\right),\left(y_{2}, y_{\alpha}\right)$, for $\alpha=3, \ldots, n$, and $\left(y_{3}, y_{\alpha}\right),\left(y_{4}, y_{\alpha}\right)$ for $\alpha=5, \ldots, n$. If we denote by $L$ the linearized operator around $u$ associated to (0.0.1), i.e.,

$$
L(\varphi):=\Delta \varphi+p \gamma|u|^{p-2} u \varphi
$$

(0.0.12)-(0.0.17) provide $N_{0}:=5(n-1)$ elements of the kernel of $L$.

We will prove that these are indeed all the elements in the kernel, i.e., solution (0.0.10) is a second example of nodal nondegenerate solution of (0.0.1). But what is more remarkable here is that if $n=4$, then $N_{0}=\mathcal{N}$, that is, the solution is maximal in the sense of Definition 0.0.2, which is the first example of a nondegenerate maximal solution in the literature, and answers the open question formulated in [20]. 
Remark 0.0.4. When $\mu \neq \lambda, h \neq k$, our solution is different from the ones constructed in [4, 5]. In [5] the symmetric case $\mu=\lambda, h=k$ is considered, which corresponds to the Clifford torus. In this case the solution has an additional symmetry which reduces the problem to one dimensional. Because of this symmetry the rank of the solutions constructed in [5] can be shown to be strictly less than $\mathcal{N}$. Thus our solutions are new. Our construction can be considered as a sort of desingularization of Clifford torus. For geometric application of desingularization of Clifford torus, we refer to the recent papers [1, 14] and the references therein.

Remark 0.0.5. The construction can be extended to higher even dimensions, that is, one can anagolously set bubbles in the $\left(y_{5}, y_{6}\right),\left(y_{7}, y_{8}\right), \ldots$, planes, in such a way that the solution is expected to be nondegenerate and the elements corresponding to the invariances generate a space of dimension exactly $\mathcal{N}$. Therefore, this type of construction presumably provides a sequence of nodal nondegenerate and maximal rank solutions of (0.0.1) for any even dimension $n \geqslant 4$. The existence of a maximal solution for odd dimensions is still an open question.

Remark 0.0.6. Nondegenerate solutions to (0.0.1) play an important role in the analysis of possible singularity formations in energy-critical wave equations. We refer to [7, 8, 9, 15, 16, 18] and the references therein.

Remark 0.0.7. The existence of sign-changing solutions for critical exponents in other contexts has been studied in [13, 22, 23].

Remark 0.0.8. There exist many works on the uniqueness and nondegeneracy of positive solutions to semilinear equations, whether or not for classical nonlinear Schrodinger equations [19] or for nonlinear fractional equations [10, 11]. The rank of the positive solutions is at most $n+1$. For sign-changing solutions the nondegeneracy question is in general quite difficult without knowing the precise behavior of the solution. Our result is the first of the type for sign-changing solutions with maximal rank.

Along the work we will denote points $y \in \mathbb{R}^{n}, n \geqslant 4$, as

$$
\begin{gathered}
y=(\bar{y}, \hat{y}), \bar{y}:=\left(y_{1}, y_{2}\right), \hat{y}:=\left(y_{3}, y_{4}\right), \text { if } n=4, \\
y=\left(\bar{y}, \hat{y}, y^{\prime}\right), \bar{y}:=\left(y_{1}, y_{2}\right), \hat{y}:=\left(y_{3}, y_{4}\right), y^{\prime}:=\left(y_{5}, \ldots, y_{n}\right), \text { if } n \geqslant 5,
\end{gathered}
$$

and we will work with the norms

$$
\|h\|_{* *}:=\left\|(1+|y|)^{n+2-\frac{2 n}{q}} h\right\|_{L^{q}\left(\mathbb{R}^{n}\right)}, \quad\|\phi\|_{*}:=\left\|\left(1+|y|^{n-2}\right) \phi\right\|_{L^{\infty}\left(\mathbb{R}^{n}\right)},
$$

where $\frac{n}{2}<q<n$ is a fixed number.

Part 1 of the paper is devoted to prove that (0.0.10) solves (0.0.1), and Part 2 concerns the proof of its nondegeneracy.

\section{Part 1. Construction of the solution}

To prove that (0.0.10) is a solution of (0.0.1) we use a Lyapunov-Schmidt reduction method, following the ideas of [4]. We linearize the equation around a first approximation and take advantage of the invertibility tools available for this setting. Then, performing a careful analysis of the error of the approximation and of the non linear terms we solve the problem by a fixed point argument. Let us point out that the precise scaling of the parameters $\mu$ and $\lambda$ plays a fundamental role here.

Recalling the definitions given in (0.0.8), (0.0.7) and (0.0.11), the main result of this part can be stated as follows. 
Theorem 1.0.1. Let $n \geqslant 4$, and let $k, h$ be positive integers so that $k=O(h)$. Then, for sufficiently large $k$ and $h$ there is a finite energy solution of the form

$$
u_{k, h}(x)=U(x)-\sum_{j=1}^{k} U_{\mu, \xi_{j}}(x)-\sum_{l=1}^{h} U_{\lambda, \eta_{l}}(x)+o_{k}(1)+o_{h}(1),
$$

where $o_{k}(1)$ and $o_{h}(1)$ denote quantities that tend to zero when $k$ and $h$ tend to infinity respectively.

\subsection{ERROR OF THE APPROXIMATION}

Denote

$$
U_{*}(y):=U(y)-\sum_{j=1}^{k} U_{\mu, \xi_{j}}(y)-\sum_{l=1}^{h} U_{\lambda, \eta_{l}}(y),
$$

and suppose that the solution $u$ we are looking for has the form

$$
u=U_{*}+\phi,
$$

where $\phi$ is a small function when compared with $U_{*}$. Then solving equation (0.0.1) is equivalent to find $\phi$ such that

$$
\Delta \phi+p \gamma\left|U_{*}\right|^{p-1} \phi+E+\gamma N(\phi)=0
$$

where

$$
\begin{aligned}
E & :=\Delta U_{*}+\gamma\left|U_{*}\right|^{p-1} U_{*}, \\
N(\phi) & :=\left|U_{*}+\phi\right|^{p-1}\left(U_{*}+\phi\right)-\left|U_{*}\right|^{p-1} U_{*}-p\left|U_{*}\right|^{p-1} \phi .
\end{aligned}
$$

In this section we try to estimate the error term $E$. In particular,

$$
\begin{aligned}
\gamma^{-1} E= & \left|U-\sum_{j=1}^{k} U_{\mu, \xi_{j}}-\sum_{l=1}^{h} U_{\lambda, \eta_{l}}\right|^{p-1}\left(U-\sum_{j=1}^{k} U_{\mu, \xi_{j}}-\sum_{l=1}^{h} U_{\lambda, \eta_{l}}\right) \\
& -U^{p}-\sum_{j=1}^{k} U_{\mu, \xi_{j}}^{p}-\sum_{l=1}^{h} U_{\lambda, \eta_{l}}^{p}
\end{aligned}
$$

We divide the study of the error in three diffentent regions. Roughly speaking, we will estimate first the $\|\cdot\|_{* *}$ norm of the error far from the points $\xi_{j}$ and $\eta_{l}$, then around $\xi_{j}$, and finally around $\eta_{l}$, for any $j=1, \ldots, k$ and $l=1, \ldots, h$. Indeed, let $\bar{\alpha}$ and $\hat{\alpha}$ be positive numbers independent of $k$ and $h$.

Exterior region: $y \in\left\{\cap_{j=1}^{k}\left\{\left|y-\xi_{j}\right|>\frac{\bar{\alpha}}{k}\right\}\right\} \cap\left\{\cap_{l=1}^{h}\left\{\left|y-\eta_{l}\right|>\frac{\hat{\alpha}}{h}\right\}\right\}$. 
For $y$ in this region we can estimate

$$
\begin{aligned}
|E| \leqslant & C\left[\frac{1}{\left(1+|y|^{2}\right)^{2}}+\left|\sum_{j=1}^{k} \frac{\mu^{\frac{n-2}{2}}}{\left|y-\xi_{j}\right|^{n-2}}\right|^{\frac{4}{n-2}}+\left|\sum_{l=1}^{h} \frac{\lambda^{\frac{n-2}{2}}}{\left|y-\eta_{l}\right|^{n-2}}\right|^{\frac{4}{n-2}}\right] \\
& \cdot\left(\sum_{j=1}^{k} \frac{\mu^{\frac{n-2}{2}}}{\left|y-\xi_{j}\right|^{n-2}}+\sum_{l=1}^{h} \frac{\lambda^{\frac{n-2}{2}}}{\left|y-\eta_{l}\right|^{n-2}}\right) \\
\leqslant & \frac{C}{\left(1+|y|^{2}\right)^{2}}\left[\sum_{j=1}^{k} \frac{\mu^{\frac{n-2}{2}}}{\left|y-\xi_{j}\right|^{n-2}}+\sum_{l=1}^{h} \frac{\lambda^{\frac{n-2}{2}}}{\left|y-\eta_{l}\right|^{n-2}}\right],
\end{aligned}
$$

where in the last inequality we have used that here

$$
\sum_{j=1}^{k} \frac{\mu^{\frac{n-2}{2}}}{\left|y-\xi_{j}\right|^{n-2}} \leqslant C\left(1-\frac{k-1}{k^{n-2}}\right), \quad \sum_{l=1}^{h} \frac{\lambda^{\frac{n-2}{2}}}{\left|y-\eta_{l}\right|^{n-2}} \leqslant C\left(1-\frac{h-1}{h^{n-2}}\right) .
$$

Thus

$$
\begin{gathered}
\left\|(1+|y|)^{n+2-\frac{2 n}{q}} E\right\|_{L^{q}\left(\left\{\cap_{j=1}^{k}\left\{\left|y-\xi_{j}\right|>\frac{\bar{\alpha}}{k}\right\}\right\} \cap\left\{\cap_{l=1}^{h}\left\{\left|y-\eta_{l}\right|>\frac{\hat{\alpha}}{h}\right\}\right\}\right)} \\
\leqslant C\left(\frac{\mu^{\frac{n-2}{2}} k^{n-2}}{k^{\frac{n}{q}-1}}+\frac{\lambda^{\frac{n-2}{2}} h^{n-2}}{h^{\frac{n}{q}-1}}\right) \\
\leqslant C\left(k^{1-\frac{n}{q}}+h^{1-\frac{n}{q}}\right) .
\end{gathered}
$$

Interior regions around $\xi_{j}: y \in\left\{\left|y-\xi_{j}\right|<\frac{\bar{\alpha}}{k}\right\}$ for some $j=1, \ldots, k$.

Let $j$ be fixed. For some $s \in(0,1)$ we have

$$
\begin{aligned}
\gamma^{-1} E= & p\left(U_{\mu, \xi_{j}}+s\left(-\sum_{i \neq j} U_{\mu, \xi_{i}}+U-\sum_{l=1}^{h} U_{\lambda, \eta_{l}}\right)\right)^{p-1}\left(-\sum_{i \neq j} U_{\mu, \xi_{i}}+U-\sum_{l=1}^{h} U_{\lambda, \eta_{l}}\right) \\
& -U^{p}-\sum_{i \neq j} U_{\mu, \xi_{i}}^{p}-\sum_{l=1}^{h} U_{\lambda, \eta_{l}}^{p} .
\end{aligned}
$$

Let us define

$$
\bar{E}_{j}(y):=\mu^{\frac{n+2}{2}} E\left(\xi_{j}+\mu y\right), \quad|y|<\frac{\bar{\alpha}}{\mu k} .
$$


Thus,

$$
\begin{aligned}
\gamma^{-1} \bar{E}_{j}(y)= & p\left(-U(y)+s\left(-\sum_{i \neq j} U\left(y-\mu^{-1}\left(\xi_{j}-\xi_{i}\right)\right)+\mu^{\frac{n-2}{2}} U\left(\xi_{j}+\mu y\right)\right.\right. \\
& -\sum_{l=1}^{h} \mu^{\frac{n-2}{2}} \lambda^{-\frac{n-2}{2}} U\left(\lambda^{-1}\left(\xi_{j}+\mu y-\eta_{l}\right)\right)\left(-\sum_{i \neq j} U\left(y-\mu^{-1}\left(\xi_{j}-\xi_{i}\right)\right)\right. \\
& +\mu^{\frac{n-2}{2}} U\left(\xi_{j}+\mu y\right)-\sum_{l=1}^{h} \mu^{\frac{n-2}{2}} \lambda^{-\frac{n-2}{2}} U\left(\lambda^{-1}\left(\xi_{j}+\mu y-\eta_{l}\right)\right) \\
& -\mu^{\frac{n+2}{2}} U^{p}\left(\xi_{j}+\mu y\right)-\sum_{i \neq j} U^{p}\left(y-\mu^{-1}\left(\xi_{i}-\xi_{j}\right)\right) \\
& -\sum_{l=1}^{h} \mu^{\frac{n+2}{2}} \lambda^{-\frac{n+2}{2}} U^{p}\left(\lambda^{-1}\left(\xi_{j}+\mu y-\eta_{l}\right),\right.
\end{aligned}
$$

and consequently

$$
\begin{aligned}
\left|\bar{E}_{j}(y)\right| & \leqslant C\left[\frac{(k \mu)^{n-2}+(\mu \lambda)^{\frac{n-2}{2}} h}{1+|y|^{4}}+\mu^{\frac{n+2}{2}}+(\mu \lambda)^{\frac{n+2}{2}} h^{\frac{n+2}{n-2}}\right] \\
& \leqslant C\left[\frac{\mu^{\frac{n-2}{2}}}{1+|y|^{4}}+\mu^{\frac{n+2}{2}}\right] .
\end{aligned}
$$

Noticing that $h=O(k)$ we can compute

$$
\left\|(1+|y|)^{n+2-\frac{2 n}{q}} \bar{E}_{j}\right\|_{L^{q}\left(|y|<\frac{\bar{\alpha}}{\mu k}\right)} \leqslant C \mu^{\frac{n}{2 q}} \leqslant C k^{-\frac{n}{q}} .
$$

Interior regions around $\eta_{l}: y \in\left\{\left|y-\eta_{l}\right|<\frac{\hat{\alpha}}{h}\right\}$ for some $l=1, \ldots, h$.

The estimates in this region follow analogously to the previous case, but interchanging the role of $\mu, k$ and $\lambda, h$. Thus, considering

$$
\hat{E}_{l}(y):=\lambda^{\frac{n+2}{2}} E\left(\eta_{l}+\mu y\right), \quad|y|<\frac{\hat{\alpha}}{\lambda h},
$$

we get

$$
\begin{aligned}
\left|\hat{E}_{l}(y)\right| & \leqslant C\left[\frac{(h \lambda)^{n-2}+(\mu \lambda)^{\frac{n-2}{2}} k}{1+|y|^{4}}+\lambda^{\frac{n+2}{2}}+(\mu \lambda)^{\frac{n+2}{2}} k^{\frac{n+2}{n-2}}\right] \\
& \leqslant C\left[\frac{\lambda^{\frac{n-2}{2}}}{1+|y|^{4}}+\lambda^{\frac{n+2}{2}}\right]
\end{aligned}
$$

and therefore

$$
\left\|(1+|y|)^{n+2-\frac{2 n}{q}} \hat{E}_{l}\right\|_{L^{q}\left(|y|<\frac{\hat{\alpha}}{\lambda h}\right)} \leqslant C \lambda^{\frac{n}{2 q}} \leqslant C h^{-\frac{n}{q}} .
$$




\subsection{Building The SOlution}

Recall from Section 1.1 that to find a solution to (0.0.1) we will prove the existence of a function $\phi$ that solves (1.1.1). We will try to build this function in a special form.

Let $\zeta(s)$ be a smooth function such that $\zeta(s)=1$ for $s>1$ and $\zeta(s)=0$ for $s>2$, and let $\bar{\alpha}, \hat{\alpha}>0$ be fixed numbers independent of $k$ and $h$. Define

$$
\begin{aligned}
& \bar{\zeta}_{j}(y):=\left\{\begin{array}{l}
\zeta\left(\left.k \bar{\alpha}^{-1}|y|^{-2}\left|y-\xi_{j}\right| y\right|^{2} \mid\right) \text { if }|y|>1, \\
\zeta\left(k \bar{\alpha}^{-1}\left|y-\xi_{j}\right|\right) \text { if }|y| \leqslant 1,
\end{array}\right. \\
& \hat{\zeta}_{l}(y):=\left\{\begin{array}{l}
\zeta\left(\left.h \hat{\alpha}^{-1}|y|^{-2}\left|y-\eta_{l}\right| y\right|^{2} \mid\right) \text { if }|y|>1, \\
\zeta\left(h \hat{\alpha}^{-1}\left|y-\eta_{l}\right|\right) \text { if }|y| \leqslant 1 .
\end{array}\right.
\end{aligned}
$$

A function of the form

$$
\phi=\sum_{j=1}^{k} \bar{\phi}_{j}+\sum_{l=1}^{h} \hat{\phi}_{l}+\psi
$$

is a solution of (1.1.1) if we solve the system

$$
\begin{gathered}
\Delta \bar{\phi}_{j}+p \gamma\left|U_{*}\right|^{p-1} \bar{\zeta}_{j} \bar{\phi}_{j}+\bar{\zeta}_{j}\left[p \gamma\left|U_{*}\right|^{p-1} \psi+E+\gamma N(\phi)\right]=0, ; j=1, \ldots, k, \\
\Delta \hat{\phi}_{l}+p \gamma\left|U_{*}\right|^{p-1} \hat{\zeta}_{l} \hat{\phi}_{l}+\hat{\zeta}_{l}\left[p \gamma\left|U_{*}\right|^{p-1} \psi+E+\gamma N(\phi)\right]=0, \quad l=1, \ldots, h, \\
\Delta \psi+p \gamma U^{p-1} \psi+\left[p \gamma\left(\left|U_{*}\right|^{p-1}-U^{p-1}\right)\left(1-\sum_{j=1}^{k} \bar{\zeta}_{j}-\sum_{l=1}^{h} \hat{\zeta}_{l}\right)\right. \\
\left.+p \gamma U^{p-1}\left(\sum_{j=1}^{k} \bar{\zeta}_{j}+\sum_{l=1}^{h} \hat{\zeta}_{l}\right)\right] \psi+p \gamma\left|U_{*}\right|^{p-1} \sum_{j=1}^{k}\left(1-\bar{\zeta}_{j}\right) \bar{\phi}_{j} \\
+p \gamma\left|U_{*}\right|^{p-1} \sum_{l=1}^{h}\left(1-\hat{\zeta}_{l}\right) \hat{\phi}_{l}+\left(1-\sum_{j=1}^{k} \bar{\zeta}_{j}-\sum_{l=1}^{h} \hat{\zeta}_{l}\right)(E+\gamma N(\phi))=0 .
\end{gathered}
$$

We assume in addition the following symmetry properties on $\bar{\phi}_{j}$ and $\hat{\phi}_{l}$,

$$
\bar{\phi}_{j}\left(\bar{y}, \hat{y}, y^{\prime}\right)=\bar{\phi}_{1}\left(e^{-\frac{2 \pi(j-1)}{k} i} \bar{y}, \hat{y}, y^{\prime}\right), \quad j=1, \ldots k,
$$

where

$$
\begin{aligned}
& \bar{\phi}_{1}\left(y_{1}, \ldots, y_{j}, \ldots, y_{n}\right)=\bar{\phi}_{1}\left(y_{1}, \ldots,-y_{j}, \ldots, y_{n}\right), \quad j=2,4,5, \ldots, n, \\
& \bar{\phi}_{1}(y)=|y|^{2-n} \bar{\phi}_{1}\left(|y|^{-2} y\right), \\
& \bar{\phi}_{1}\left(\bar{y}, \hat{y}, y^{\prime}\right)=\bar{\phi}_{1}\left(\bar{y}, e^{\frac{2 \pi(l-1)}{h} i} \hat{y}, y^{\prime}\right), \quad l=1, \ldots, h,
\end{aligned}
$$

and

where

$$
\hat{\phi}_{j}\left(\bar{y}, \hat{y}, y^{\prime}\right)=\hat{\phi}_{1}\left(\bar{y}, e^{-\frac{2 \pi(l-1)}{h} i} \hat{y}, y^{\prime}\right), \quad l=1, \ldots h
$$

$$
\begin{aligned}
& \hat{\phi}_{1}\left(y_{1}, \ldots, y_{j}, \ldots, y_{n}\right)=\hat{\phi}_{1}\left(y_{1}, \ldots,-y_{j}, \ldots, y_{n}\right), \quad j=2,4,5, \ldots, n, \\
& \hat{\phi}_{1}(y)=|y|^{2-n} \hat{\phi}_{1}\left(|y|^{-2} y\right), \\
& \hat{\phi}_{1}\left(\bar{y}, \hat{y}, y^{\prime}\right)=\hat{\phi}_{1}\left(e^{\frac{2 \pi(j-1)}{k} i} \bar{y}, \hat{y}, y^{\prime}\right), \quad j=1, \ldots, k .
\end{aligned}
$$


Assume in addition that

$$
\begin{array}{ll}
\left\|\overline{\bar{\phi}}_{1}\right\|_{*} \leqslant \rho, & \overline{\bar{\phi}}_{1}(y):=\mu^{\frac{n-2}{2}} \bar{\phi}_{1}\left(\xi_{1}+\mu y\right), \\
\left\|\hat{\hat{\phi}}_{1}\right\|_{*} \leqslant \rho, & \hat{\hat{\phi}}_{1}(y):=\lambda^{\frac{n-2}{2}} \hat{\phi}_{1}\left(\eta_{1}+\lambda y\right),
\end{array}
$$

for $\rho>0$ small.

Lemma 1.2.1. There exist constants $k_{0}, h_{0}, C, \rho_{0}$ such that, for all $k \geqslant k_{0}$ and $h \geqslant h_{0}$, if $\bar{\phi}_{j}$, $j=1, \ldots, k$, and $\hat{\phi}_{l}, l=1, \ldots, h$ satisfy conditions (2.2.16)-(2.2.20) with $\rho<\rho_{0}$ then there exists a unique solution $\psi=\Psi\left(\overline{\bar{\phi}}_{1}, \hat{\hat{\phi}}_{1}\right)$ to equation (1.2.4), that satisfies the symmetries

$$
\begin{gathered}
\psi\left(y_{1}, \ldots, y_{\alpha}, \ldots\right)=\psi\left(y_{1}, \ldots,-y_{\alpha}, \ldots\right), \quad \alpha=5, \ldots, n, \\
\psi\left(\bar{y}, \hat{y}, y^{\prime}\right)=\psi\left(e^{\frac{2 \pi(j-1)}{k} i \bar{y}}, \hat{y}, y^{\prime}\right), \quad j=1, \ldots, k, \\
\psi\left(\bar{y}, \hat{y}, y^{\prime}\right)=\psi\left(\bar{y}, e^{\frac{2 \pi(l-1)}{h} i} \hat{y}, y^{\prime}\right), \quad l=1, \ldots, h, \\
\psi(y)=|y|^{2-n} \psi\left(|y|^{-2} y\right),
\end{gathered}
$$

and such that

$$
\|\psi\|_{*} \leqslant C\left[\left\|\overline{\bar{\phi}}_{1}\right\|_{*}+\left\|\hat{\hat{\phi}}_{1}\right\|_{*}+k^{1-\frac{n}{q}}+h^{1-\frac{n}{q}}\right] .
$$

Moreover, the operator $\Psi$ satisfies

$$
\left\|\Psi\left(\overline{\bar{\phi}}_{1}^{1}, \hat{\hat{\phi}}_{1}^{1}\right)-\Psi\left(\overline{\bar{\phi}}_{1}^{2}, \hat{\hat{\phi}}_{1}^{2}\right)\right\|_{*} \leqslant C\left(\left\|\overline{\bar{\phi}}_{1}^{1}-\overline{\bar{\phi}}_{1}^{2}\right\|_{*}+\left\|\hat{\hat{\phi}}_{1}^{1}-\hat{\hat{\phi}}_{1}^{2}\right\|_{*}\right) .
$$

Proof. We write equation (1.2.4) as

$$
\Delta \psi+p \gamma U^{p-1} \psi+V(y) \psi+p \gamma\left|U_{*}\right|^{p-1}\left(\sum_{j=1}^{k}\left(1-\bar{\zeta}_{j}\right) \bar{\phi}_{j}+\sum_{l=1}^{h}\left(1-\hat{\zeta}_{l}\right) \hat{\phi}_{l}\right)+M(\psi)=0,
$$

where

$$
\begin{aligned}
V(y) & :=p \gamma\left(\left|U_{*}\right|^{p-1}-U^{p-1}\right)\left(1-\sum_{j=1}^{k} \bar{\zeta}_{j}-\sum_{l=1}^{h} \hat{\zeta}_{l}\right)+p \gamma U^{p-1}\left(\sum_{j=1}^{k} \bar{\zeta}_{j}+\sum_{l=1}^{h} \hat{\zeta}_{l}\right), \\
& =: V_{1}(y)+V_{2}(y),
\end{aligned}
$$

and

$$
M(\psi):=\left(1-\sum_{j=1}^{k} \bar{\zeta}_{j}-\sum_{l=1}^{h} \hat{\zeta}_{l}\right)(E+\gamma N(\phi))=0 .
$$

Consider first the problem

$$
\Delta \psi+p \gamma U^{p-1} \psi=h
$$

where $h$ is a function satisfying (1.2.10)-(1.2.12), $\|h\|_{* *}<+\infty$ and

$$
h(y)=|y|^{-n-2} h\left(|y|^{-2} y\right) .
$$

Let

$$
Z_{\alpha}:=\partial_{y_{\alpha}} U, \quad \alpha=1, \cdots, n \text { and } Z_{0}=y \cdot \nabla U+\frac{n-2}{2} U .
$$

Due to the oddness of $Z_{\alpha}$ and assumption (1.2.10) on $h$ it yields

$$
\int_{\mathbb{R}^{n}} Z_{\alpha} h=0 \text { for all } \alpha=5, \cdots, n .
$$


The cases $\alpha=0,1,2,3,4$ also vanish proceeding as in the proof of [4, Lemma 4.1] as a consequence of (1.2.11)-(1.2.12). Thus we can apply the linear existence result [4, Lemma 3.1] to ensure the existence of a unique solution $\psi$ to (1.2.17) such that

$$
\int_{\mathbb{R}^{n}} U^{p-1} Z_{\alpha} \psi=0 \text { for all } \alpha=0,1, \ldots, n,
$$

and $\|\psi\|_{*} \leqslant C\|h\|_{* *}$. Notice in addition that the functions

$$
\begin{aligned}
\psi_{\alpha}(y) & :=\psi\left(\bar{y}, \hat{y}, \ldots,-y_{i}, \ldots, y_{n}\right), \quad \alpha=5, \cdots, n, \\
\psi_{12 j}(y) & :=\psi\left(e^{\left.\frac{2 \pi(j-1)}{k} i \bar{y}, \hat{y}, y^{\prime}\right), \quad j=1, \ldots, k,}\right. \\
\psi_{34 l}(y) & :=\psi\left(\bar{y}, e^{\frac{2 \pi(l-1)}{h} i} \hat{y}, y^{\prime}\right), \quad l=1, \ldots, h, \\
\psi_{n+1}(y) & :=|y|^{2-n} \psi\left(|y|^{-2} y\right),
\end{aligned}
$$

also satisfy (1.2.17) and thus, by the uniqueness,

$$
\psi=\psi_{\alpha}=\psi_{12 j}=\psi_{34 l}=\psi_{n+1}
$$

for all $\alpha=5, \ldots, n, j=1, \ldots, k, l=1, \ldots, h$, i.e., $\psi$ satisfies (1.2.10)-(1.2.13). Therefore, (1.2.17) has a unique bounded solution $\psi=T(h)$ satisfying symmetries (1.2.10)-(1.2.13) and

$$
\|\psi\|_{*} \leqslant C\|h\|_{* *}
$$

for a constant depending only on $q$ and $n$.

We will solve (1.2.16) by means of a fixed point argument, writting

$$
\psi=-T\left(V \psi+p \gamma\left|U_{*}\right|^{p-1}\left(\sum_{j=1}^{k}\left(1-\bar{\zeta}_{j}\right) \bar{\phi}_{j}+\sum_{l=1}^{h}\left(1-\hat{\zeta}_{l}\right) \hat{\phi}_{l}\right)+M(\psi)\right)=: \mathcal{M}(\psi),
$$

$\psi \in X$, where $X$ is the space of continuous functions $\psi$ with $\|\psi\|_{*}<+\infty$ satisfying (1.2.10)(1.2.13). Thanks to the special form of $U_{*}$ and to the symmetry assumptions on $\bar{\phi}_{j}$ and $\hat{\phi}_{l}$,

$$
V \psi+p \gamma\left|U_{*}\right|^{p-1}\left(\sum_{j=1}^{k}\left(1-\bar{\zeta}_{j}\right) \bar{\phi}_{j}+\sum_{l=1}^{h}\left(1-\hat{\zeta}_{l}\right) \hat{\phi}_{l}\right)+M(\psi)
$$

satisfies (1.2.10)-(1.2.12) and (1.2.18) if $\psi \in X$, and $\mathcal{M}$ is well defined. Actually, we claim that $\mathcal{M}$ is a contraction mapping in the \|\|$_{*}$ norm in a small ball around the origin in $X$. Indeed,

$$
\begin{aligned}
\left|V_{1}(y)\right| & \leqslant \gamma p(p-1)\left|U-s\left(\sum_{j=1}^{k} U_{\mu, \xi_{j}}+\sum_{l=1}^{h} U_{\lambda, \eta_{l}}\right)\right|^{p-2}\left(\sum_{j=1}^{k} U_{\mu, \xi_{j}}+\sum_{l=1}^{h} U_{\lambda, \eta_{l}}\right) \\
& \leqslant C U^{p-2}(y)\left(\sum_{j=1}^{k} \frac{\mu^{\frac{n-2}{2}}}{\left|y-\xi_{j}\right|^{n-2}}+\sum_{l=1}^{h} \frac{\lambda^{\frac{n-2}{2}}}{\left|y-\eta_{l}\right|^{n-2}}\right),
\end{aligned}
$$

and thus

$$
\left|V_{1} \psi(y)\right| \leqslant C\|\psi\|_{*} U^{p-1}(y)\left(\sum_{j=1}^{k} \frac{\mu^{\frac{n-2}{2}}}{\left|y-\xi_{j}\right|^{n-2}}+\sum_{l=1}^{h} \frac{\lambda^{\frac{n-2}{2}}}{\left|y-\eta_{l}\right|^{n-2}}\right) .
$$

Proceeding as in (1.1.3) we get

$$
\left\|V_{1} \psi\right\|_{* *} \leqslant C\|\psi\|_{*}\left(k^{1-\frac{n}{q}}+h^{1-\frac{n}{q}}\right) .
$$


On the other hand,

$$
\begin{aligned}
\left\|V_{2} \psi\right\|_{* *} & \leqslant \sum_{j=1}^{k}\left\|p U^{p-1} \psi \bar{\zeta}_{j}\right\|_{* *}+\sum_{l=1}^{h}\left\|p U^{p-1} \psi \hat{\zeta}_{l}\right\|_{* *} \\
& \leqslant C\|\psi\|_{*}\left(k^{1-\frac{n}{q}}+h^{1-\frac{n}{q}}\right),
\end{aligned}
$$

and putting together (1.2.20) and (1.2.21) we conclude

$$
\|V \psi\|_{* *} \leqslant C\|\psi\|_{*}\left(k^{1-\frac{n}{q}}+h^{1-\frac{n}{q}}\right) .
$$

Assume $\left|y-\xi_{j}\right|>\frac{\bar{\alpha}}{2 k}$ and $\left|y-\eta_{l}\right|>\frac{\hat{\alpha}}{2 h}$ for all $j$ and $l$. We knew that in this region

$$
\|E\|_{* *} \leqslant C\left(k^{1-\frac{n}{q}}+h^{1-\frac{n}{q}}\right) .
$$

Moreover,

$$
\left|N\left(\sum_{j=1}^{k} \bar{\phi}_{j}+\sum_{l=1}^{h} \hat{\phi}_{l}+\psi\right)\right| \leqslant C U^{p-2}\left(\left|\sum_{j=1}^{k} \bar{\phi}_{j}\right|^{2}+\left|\sum_{l=1}^{h} \hat{\phi}_{l}\right|^{2}+|\psi|^{2}\right) .
$$

From (2.2.20), we get

$$
\left|\bar{\phi}_{j}(y)\right| \leqslant C\left\|\overline{\bar{\phi}}_{1}\right\|_{*} \frac{\mu^{\frac{n-2}{2}}}{\mu^{n-2}+\left|y-\xi_{j}\right|^{n-2}}, \quad\left|\hat{\phi}_{l}(y)\right| \leqslant C\left\|\hat{\hat{\phi}}_{1}\right\|_{*} \frac{\lambda^{\frac{n-2}{2}}}{\lambda^{n-2}+\left|y-\eta_{l}\right|^{n-2}} .
$$

Moreover,

$$
U^{p-2}|\psi|^{2} \leqslant U^{p}\|\psi\|_{*}^{2}
$$

Hence, proceeding again as in (1.1.3), we obtain

$$
\|M(\psi)\|_{* *} \leqslant C k^{1-\frac{n}{q}}\left(1+\left\|\overline{\bar{\phi}}_{1}\right\|_{*}^{2}\right)+C h^{1-\frac{n}{q}}\left(1+\left\|\hat{\hat{\phi}}_{1}\right\|_{*}^{2}\right)+C\|\psi\|_{*}^{2} .
$$

Likewise, if $\left|y-\xi_{j}\right|>\frac{\bar{\alpha}}{2 k}$ and $\left|y-\eta_{l}\right|>\frac{\hat{\alpha}}{2 h}$,

$$
\left\|\left|U_{*}\right|^{p-1}\left(\sum_{j=1}^{k} \bar{\phi}_{j}+\sum_{l=1}^{h} \hat{\phi}_{l}\right)\right\|_{* *} \leqslant C k^{1-\frac{n}{q}}\left\|\overline{\bar{\phi}}_{1}\right\|_{*}+C h^{1-\frac{n}{q}}\left\|\hat{\hat{\phi}}_{1}\right\|_{*}
$$

Moreover, for $\psi_{1}, \psi_{2}$ satisfying $\left\|\psi_{1}\right\|<\rho,\left\|\psi_{2}\right\|<\rho$ it follows

$$
\left\|M\left(\psi_{1}\right)-M\left(\psi_{2}\right)\right\|_{* *} \leqslant C \rho\left\|\psi_{1}-\psi_{2}\right\|_{*} .
$$

Joining (1.2.22), (1.2.23) and (1.2.24), we see that for $\rho$ small enough the operator $\mathcal{M}$ defines a contraction map in the set of functions $\psi \in X$ with

$$
\|\psi\|_{*} \leqslant C\left[\left\|\overline{\bar{\phi}}_{1}\right\|_{*}+k^{1-\frac{n}{q}}+\left\|\hat{\hat{\phi}}_{1}\right\|_{*}+h^{1-\frac{n}{q}}\right], \quad\left\|\overline{\bar{\phi}}_{1}\right\|_{*}<\rho, \quad\left\|\hat{\hat{\phi}}_{1}\right\|_{*}<\rho,
$$

for $\rho$ small. Therefore, there exists a solution of (1.2.16) satisfying conditions (1.2.10)-(1.2.14). The Lipschitz condition (1.2.15) easily follows.

Consider the operator $\Psi\left(\overline{\bar{\phi}}_{1}, \hat{\hat{\phi}}_{1}\right)$. Equations (2.2.7) and (2.2.8) reduce to solve one of each, for example for $\bar{\phi}_{1}$ and $\hat{\phi}_{1}$.

We try to solve first

$$
\Delta \bar{\phi}_{1}+p \gamma\left|U_{*}\right|^{p-1} \bar{\zeta}_{1} \bar{\phi}_{1}+\bar{\zeta}_{1}\left[p \gamma\left|U_{*}\right|^{p-1} \Psi\left(\overline{\bar{\phi}}_{1}, \hat{\hat{\phi}}_{1}\right)+E+\gamma N(\phi)\right]=0 \text { in } \mathbb{R}^{n}
$$


or equivalently,

$$
\Delta \bar{\phi}_{1}+p \gamma\left|U_{\mu, \xi_{1}}\right|^{p-1} \bar{\phi}_{1}+\bar{\zeta}_{1} E+\gamma \overline{\mathcal{N}}\left(\overline{\bar{\phi}}_{1}, \hat{\hat{\phi}}_{1}\right)=0
$$

where

$$
\overline{\mathcal{N}}\left(\overline{\bar{\phi}}_{1}, \hat{\hat{\phi}}_{1}\right):=p\left(\left|U_{*}\right|^{p-1} \bar{\zeta}_{1}-\left|U_{\mu, \xi_{1}}\right|^{p-1}\right) \bar{\phi}_{1}+\bar{\zeta}_{1}\left[p\left|U_{*}\right|^{p-1} \Psi\left(\overline{\bar{\phi}}_{1}, \hat{\hat{\phi}}_{1}\right)+N(\phi)\right] .
$$

Consider first a general function $\bar{h}$ and the problem

$$
\Delta \bar{\phi}+p \gamma U_{\mu, \xi_{1}}^{p-1} \bar{\phi}+\bar{h}=\bar{c}_{0} U_{\mu, \xi_{1}}^{p-1} \bar{Z}_{0} \text { in } \mathbb{R}^{n}
$$

where

$$
\bar{Z}_{0}(y):=\mu^{-\frac{n-2}{2}} Z_{0}\left(\frac{y-\xi_{1}}{\mu}\right), \quad \bar{c}_{0}:=\frac{\int_{\mathbb{R}^{n}} \bar{h} \bar{Z}_{0}}{\int_{\mathbb{R}^{n}} U_{\mu, \xi_{1}}^{p-1} \bar{Z}_{0}^{2}},
$$

with $Z_{0}$ defined in (1.2.19).

Lemma 1.2.2. Suppose that $\bar{h}$ is even with respect to each of the variables $y_{2}, y_{4}, y_{5}, \ldots, y_{n}$ and such that

$$
\bar{h}(y)=|y|^{-n-2} \bar{h}\left(|y|^{-2} y\right), \quad \bar{h}(y)=\bar{h}\left(\bar{y}, e^{\frac{2 \pi(l-1)}{h}} \hat{y}, y^{\prime}\right), \quad l=1, \ldots, h .
$$

Assume that

$$
h(y):=\mu^{\frac{n+2}{2}} \bar{h}\left(\xi_{1}+\mu y\right)
$$

satisfies $\|h\|_{* *}<+\infty$. Then problem (1.2.26) has a unique solution $\bar{\phi}:=\bar{T}(\bar{h})$ that is even with respect to the variables $y_{2}, y_{4}, y_{5}, \ldots, y_{n}$, invariant under Kelvin's tranform, i.e.,

$$
\bar{\phi}(y)=|y|^{2-n} \bar{\phi}\left(|y|^{-2} y\right)
$$

and with $\overline{\bar{\phi}}(y):=\mu^{\frac{n-2}{2}} \bar{\phi}\left(\xi_{1}+\mu y\right)$ satisfying

$$
\int_{\mathbb{R}^{n}} \overline{\bar{\phi}} U^{p-1} Z_{0}=0, \quad\|\overline{\bar{\phi}}\|_{*} \leqslant C\|h\|_{* *} .
$$

Proof. We assume with no loss of generality that

$$
\int_{\mathbb{R}^{n}} \bar{h} \bar{Z}_{0}=0, \quad \text { i.e., } \quad \bar{c}_{0}=0 .
$$

Thus, equation (1.2.26) is equivalent to

$$
\Delta \overline{\bar{\phi}}+p \gamma|U|^{p-1} \overline{\bar{\phi}}=-h \quad \text { in } \mathbb{R}^{n} .
$$

Due to the evenness of $h$ we know that

$$
\int_{\mathbb{R}^{n}} h Z_{\alpha}=0, \quad \alpha=2,4,5, \cdots, n
$$

The proof of

$$
\int_{\mathbb{R}^{n}} h Z_{1}=0
$$

follows exactly as in the proof of [4, Lemma 4.2], so we focus on the case $\alpha=3$.

Indeed, denote by $w_{\mu}(y):=\mu^{-\frac{n-2}{2}} U\left(\mu^{-1} y\right)$, and $J(t):=\int_{\mathbb{R}^{n}} w_{\mu}\left(y-\xi_{1}+t e_{3}\right) \bar{h}(y) d y$. Notice first that

$$
\left.\frac{d}{d t} J(t)\right|_{t=0}=\int_{\mathbb{R}^{n}} \partial_{y_{3}} w_{\mu}\left(y-\xi_{1}\right) \bar{h}(y) d y=\int_{\mathbb{R}^{n}} h Z_{3}
$$


On the other hand, defining $\tilde{y}:=\left(\bar{y}, e^{\frac{2 \pi(l-1)}{h}} \hat{y}, y^{\prime}\right)$ for some $l=2,3, \ldots, h$, it can be checked that

$$
\left|\tilde{y}-\xi_{1}+t e_{3}\right|^{2}=\left|y-\xi_{1}+t \tilde{e}\right|^{2}, \quad t \in \mathbb{R}^{n},
$$

where $\tilde{e}:=\left(0,0, \cos \left(\frac{2 \pi(l-1)}{h}\right),-\sin \left(\frac{2 \pi(l-1)}{h}\right), 0, \ldots\right)$. Thus, after a change of variables, by (1.2.27),

$$
J(t)=\int_{\mathbb{R}^{n}} w_{\mu}\left(\tilde{y}-\xi_{1}+t e_{3}\right) \bar{h}(\tilde{y}) d \tilde{y}=\int_{\mathbb{R}^{n}} w_{\mu}\left(y-\xi_{1}+t \tilde{e}\right) \bar{h}(y) d y .
$$

Differentiating here,

$$
\begin{aligned}
\left.\frac{d}{d t} J(t)\right|_{t=0}= & \cos \left(\frac{2 \pi(l-1)}{h}\right) \int_{\mathbb{R}^{n}} \partial_{y_{3}} w_{\mu}\left(y-\xi_{1}\right) \bar{h}(y) d y \\
& -\sin \left(\frac{2 \pi(l-1)}{h}\right) \int_{\mathbb{R}^{n}} \partial_{y_{4}} w_{\mu}\left(y-\xi_{1}\right) \bar{h}(y) d y \\
= & \cos \left(\frac{2 \pi(l-1)}{h}\right) \int_{\mathbb{R}^{n}} h Z_{3} d y-\sin \left(\frac{2 \pi(l-1)}{h}\right) \int_{\mathbb{R}^{n}} h Z_{4} d y .
\end{aligned}
$$

Applying (1.2.28) and (1.2.29) we conclude that necessarily $\int_{\mathbb{R}^{n}} h Z_{3}=0$. Thus, by [4, Lemma 3.1] there exists a unique solution $\overline{\bar{\phi}}$ satisfying

$$
\|\overline{\bar{\phi}}\|_{*} \leqslant C\|h\|_{* *}, \quad \int_{\mathbb{R}^{n}} \overline{\bar{\phi}} U^{p-1} Z_{n+1}=0 .
$$

The invariance under Kelvin transform and the symmetries are obtained as a consequence of the uniqueness.

Likewise, we rewrite

$$
\Delta \hat{\phi}_{1}+p \gamma\left|U_{*}\right|^{p-1} \hat{\zeta}_{1} \hat{\phi}_{1}+\hat{\zeta}_{1}\left[p \gamma\left|U_{*}\right|^{p-1} \Psi\left(\overline{\bar{\phi}}_{1}, \hat{\hat{\phi}}_{1}\right)+E+\gamma N(\phi)\right]=0 \text { in } \mathbb{R}^{n},
$$

as

$$
\Delta \hat{\phi}_{1}+p \gamma\left|U_{\lambda, \eta_{1}}\right|^{p-1} \hat{\phi}_{1}+\hat{\zeta}_{1} E+\gamma \hat{\mathcal{N}}\left(\overline{\bar{\phi}}_{1}, \hat{\hat{\phi}}_{1}\right)=0
$$

with

$$
\hat{\mathcal{N}}\left(\overline{\bar{\phi}}_{1}, \hat{\hat{\phi}}_{1}\right):=p\left(\left|U_{*}\right|^{p-1} \hat{\zeta}_{1}-\left|U_{\lambda, \eta_{1}}\right|^{p-1}\right) \hat{\phi}_{1}+\hat{\zeta}_{1}\left[p\left|U_{*}\right|^{p-1} \Psi\left(\overline{\bar{\phi}}_{1}, \hat{\hat{\phi}}_{1}\right)+N(\phi)\right],
$$

and we consider the problem

$$
\Delta \hat{\phi}+p \gamma U_{\lambda, \eta_{1}}^{p-1} \hat{\phi}+\hat{h}=\hat{c}_{0} U_{\lambda, \eta_{1}}^{p-1} \hat{Z}_{0} \text { in } \mathbb{R}^{n}
$$

where $\hat{h}$ is a general function and

$$
\hat{Z}_{0}(y):=\lambda^{-\frac{n-2}{2}} Z_{0}\left(\frac{y-\eta_{1}}{\lambda}\right), \quad \hat{c}_{0}:=\frac{\int_{\mathbb{R}^{n}} \hat{h} \hat{Z}_{0}}{\int_{\mathbb{R}^{n}} U_{\lambda, \eta_{1}}^{p-1} \hat{Z}_{0}^{2}} .
$$

Lemma 1.2.3. Suppose that $\hat{h}$ is even with respect to each of the variables $y_{2}, y_{4}, y_{5}, \ldots, y_{n}$ and such that

$$
\hat{h}(y)=|y|^{-n-2} \hat{h}\left(|y|^{-2} y\right), \quad \hat{h}(y)=\hat{h}\left(e^{\frac{2 \pi(j-1)}{k}} \bar{y}, \hat{y}, y^{\prime}\right), \quad j=1, \ldots, k .
$$

Assume that

$$
h(y):=\lambda^{\frac{n+2}{2}} \hat{h}\left(\eta_{1}+\lambda y\right)
$$


satisfies $\|h\|_{* *}<+\infty$. Then problem (1.2.31) has a unique solution $\hat{\phi}:=\hat{T}(\hat{h})$ that is even with respect to the variables $y_{2}, y_{4}, y_{5}, \ldots, y_{n}$, invariant under Kelvin's tranform, i.e.,

$$
\hat{\phi}(y)=|y|^{2-n} \hat{\phi}\left(|y|^{-2} y\right)
$$

and with $\hat{\hat{\phi}}(y):=\lambda^{\frac{n-2}{2}} \hat{\phi}\left(\eta_{1}+\lambda y\right)$ satisfying

$$
\int_{\mathbb{R}^{n}} \hat{\hat{\phi}} U^{p-1} Z_{0}=0, \quad\|\hat{\hat{\phi}}\|_{*} \leqslant C\|h\|_{* *} .
$$

The proof of this result is analogous to the one for Lemma 1.2.2, interchanging the roles of $\mu$ and $\xi_{1}$ with $\lambda$ and $\eta_{1}$, so we skip it.

We use these lemmas to solve the projected versions of (1.2.25) and (1.2.30), that is,

$$
\begin{aligned}
& \Delta \bar{\phi}_{1}+p \gamma\left|U_{\mu, \xi_{1}}\right|^{p-1} \bar{\phi}_{1}+\bar{\zeta}_{1} E+\gamma \overline{\mathcal{N}}\left(\overline{\bar{\phi}}_{1}, \hat{\hat{\phi}}_{1}\right)=\bar{c}_{0} U_{\mu, \xi_{1}}^{p-1} \bar{Z}_{0}, \\
& \Delta \hat{\phi}_{1}+p \gamma\left|U_{\lambda, \eta_{1}}\right|^{p-1} \hat{\phi}_{1}+\hat{\zeta}_{1} E+\gamma \hat{\mathcal{N}}\left(\overline{\bar{\phi}}_{1}, \hat{\hat{\phi}}_{1}\right)=\hat{c}_{0} U_{\lambda, \eta_{1}}^{p-1} \hat{Z}_{0},
\end{aligned}
$$

in $\mathbb{R}^{n}$, with

$$
\bar{c}_{0}:=\frac{\int_{\mathbb{R}^{n}}\left(\bar{\zeta}_{1} E+\gamma \overline{\mathcal{N}}\left(\overline{\bar{\phi}}_{1}, \hat{\hat{\phi}}_{1}\right)\right) \bar{Z}_{0}}{\int_{\mathbb{R}^{n}} U_{\mu, \xi_{1}}^{p-1} \bar{Z}_{0}^{2}}, \quad \hat{c}_{0}:=\frac{\int_{\mathbb{R}^{n}}\left(\hat{\zeta}_{1} E+\gamma \hat{\mathcal{N}}\left(\overline{\bar{\phi}}_{1}, \hat{\hat{\phi}}_{1}\right)\right) \hat{Z}_{0}}{\int_{\mathbb{R}^{n}} U_{\lambda, \eta_{1}}^{p-1} \hat{Z}_{0}^{2}}
$$

Proposition 1.2.4. There exists a unique solution $\phi_{1}=\left(\overline{\bar{\phi}}_{1}, \hat{\hat{\phi}}_{1}\right)=\left(\overline{\bar{\phi}}_{1}(\delta), \hat{\hat{\phi}}_{1}(\varepsilon)\right)$ to (1.2.32) such that

$$
\left\|\phi_{1}\right\|_{*}:=\left\|\overline{\bar{\phi}}_{1}\right\|_{*}+\left\|\hat{\hat{\phi}}_{1}\right\|_{*} \leqslant C\left(k^{-\frac{n}{q}}+h^{-\frac{n}{q}}\right),
$$

and

$$
\left\|\overline{\mathcal{N}}\left(\overline{\bar{\phi}}_{1}, \hat{\hat{\phi}}_{1}\right)\right\|_{* *} \leqslant C k^{-\frac{2 n}{q}}, \quad\left\|\hat{\mathcal{N}}\left(\overline{\bar{\phi}}_{1}, \hat{\hat{\phi}}_{1}\right)\right\|_{* *} \leqslant C h^{-\frac{2 n}{q}} .
$$

Proof. Denote by $\bar{T}$ and $\hat{T}$ the linear operators predicted by Lemma 1.2 .2 and Lemma 1.2 .3 respectively. Thus, solving (1.2.32) is equivalent to solve the fixed point problem

$$
\phi_{1}=\left(\begin{array}{c}
\bar{\phi}_{1} \\
\hat{\phi}_{1}
\end{array}\right)=\left(\begin{array}{c}
\bar{T}\left(\bar{\zeta}_{1} E+\gamma \overline{\mathcal{N}}\left(\overline{\bar{\phi}}_{1}, \hat{\hat{\phi}}_{1}\right)\right) \\
\hat{T}\left(\hat{\zeta}_{1} E+\gamma \hat{\mathcal{N}}\left(\overline{\bar{\phi}}_{1}, \hat{\hat{\phi}}_{1}\right)\right)
\end{array}\right)=\left(\begin{array}{c}
\overline{\mathcal{M}}\left(\overline{\bar{\phi}}_{1}, \hat{\hat{\phi}}_{1}\right) \\
\hat{\mathcal{M}}\left(\overline{\bar{\phi}}_{1}, \hat{\hat{\phi}}_{1}\right)
\end{array}\right)=: \mathcal{M}\left(\phi_{1}\right) .
$$

Let us focus first on $\overline{\mathcal{M}}\left(\overline{\bar{\phi}}_{1}, \hat{\hat{\phi}}_{1}\right):=\bar{T}\left(\bar{\zeta}_{1} E+\gamma \overline{\mathcal{N}}\left(\overline{\bar{\phi}}_{1}, \hat{\hat{\phi}}_{1}\right)\right)$. Recall that

$$
\overline{\mathcal{N}}\left(\overline{\bar{\phi}}_{1}, \hat{\hat{\phi}}_{1}\right):=p\left(\left|U_{*}\right|^{p-1} \bar{\zeta}_{1}-\left|U_{\mu, \xi_{1}}\right|^{p-1}\right) \bar{\phi}_{1}+\bar{\zeta}_{1}\left[p\left|U_{*}\right|^{p-1} \Psi\left(\overline{\bar{\phi}}_{1}, \hat{\hat{\phi}}_{1}\right)+N(\phi)\right] .
$$

Denote in general

$$
\tilde{f}(y)=\mu^{\frac{n+2}{2}} f\left(\xi_{1}+\mu y\right) .
$$

Consider

$$
f_{1}(y):=p \bar{\zeta}_{1}\left(\left|U_{*}\right|^{p-1}-\left|U_{\mu, \xi_{1}}\right|^{p-1}\right) \bar{\phi}_{1}
$$


For $|y|<\frac{\bar{\alpha}}{\mu k}$,

$$
\begin{aligned}
\left|\tilde{f}_{1}(y)\right|=\mid & p\left(\left(U(y)+\sum_{j=2}^{k} U\left(y+\mu^{-1}\left(\xi_{1}-\xi_{j}\right)\right)\right.\right. \\
& +\sum_{l=1}^{h} \mu^{\frac{n-2}{2}} \lambda^{-\frac{n-2}{2}} U\left(\lambda^{-1}\left(\xi_{1}+\mu y-\eta_{l}\right)\right) \\
& \left.\left.-\mu^{\frac{n-2}{2}} U\left(\xi_{1}+\mu y\right)\right)^{p-1}-U^{p-1}(y)\right) \overline{\bar{\phi}}_{1}(y) \mid .
\end{aligned}
$$

Noticing that

$$
\sum_{j=2}^{k} U\left(y+\mu^{-1}\left(\xi_{1}-\xi_{j}\right)\right) \leqslant C \mu^{n-2} k^{n-2} \sum_{j=1}^{k} \frac{1}{j^{n-2}} \leqslant C \mu^{\frac{n-2}{2}},
$$

and

$$
\begin{aligned}
\sum_{l=1}^{h} \mu^{\frac{n-2}{2}} \lambda^{-\frac{n-2}{2}} & U\left(\lambda^{-1}\left(\xi_{1}+\mu y-\eta_{l}\right)\right) \\
& \leqslant C \sum_{l=1}^{h} \mu^{\frac{n-2}{2}} \lambda^{-\frac{n-2}{2}} \leqslant C \mu^{\frac{n-2}{2}} \lambda^{-\frac{n-2}{2}} h \leqslant C \mu^{\frac{n-2}{2}},
\end{aligned}
$$

doing a Taylor expansion we get

$$
\left|\tilde{f}_{1}(y)\right| \leqslant C \mu^{\frac{n-2}{2}} U^{p-2}(y)\left|\overline{\bar{\phi}}_{1}(y)\right| \leqslant C \mu^{\frac{n-2}{2}} U^{p-1}(y)\left\|\overline{\bar{\phi}}_{1}\right\|_{*}
$$

and, proceeding as in the computations for the interior error,

$$
\left\|\tilde{f}_{1}\right\|_{* *} \leqslant C \mu^{\frac{n}{2 q}}\left\|\overline{\bar{\phi}}_{1}\right\|_{*} .
$$

For the term

$$
f_{2}:=\left(\bar{\zeta}_{1}-1\right) U_{\mu, \xi_{1}}^{p-1} \bar{\phi}_{1}
$$

we have

$$
\left|\tilde{f}_{2}(y)\right| \leqslant U^{p}(y)\left\|\overline{\bar{\phi}}_{1}\right\|_{*}, \quad|y|>\frac{\bar{\alpha}}{\mu k}, \quad\left\|\tilde{f}_{2}\right\|_{* *} \leqslant C \mu^{\frac{n}{2 q}}\left\|\overline{\bar{\phi}}_{1}\right\|_{*} .
$$

Consider now

$$
f_{3}:=\bar{\zeta}_{1} p\left|U_{*}\right|^{p-1} \Psi\left(\bar{\phi}_{1}, \hat{\phi}_{1}\right) .
$$

Using (1.2.34), (1.2.35) and (1.2.14) we get that, for $|y| \leqslant \frac{\bar{\alpha}}{\mu k}$,

$$
\begin{aligned}
& \left|\tilde{f}_{3}(y)\right| \leqslant C U^{p-1} \mu^{\frac{n-2}{2}}\left\|\Psi\left(\bar{\phi}_{1}, \hat{\phi}_{1}\right)\right\|_{L^{\infty}\left(\mathbb{R}^{n}\right)} \\
& \leqslant C U^{p-1} \mu^{\frac{n-2}{2}}\left(\left\|\overline{\bar{\phi}}_{1}\right\|_{*}+\left\|\hat{\hat{\phi}}_{1}\right\|_{*}+k^{1-\frac{n}{q}}+h^{1-\frac{n}{q}}\right), \\
& \left\|\tilde{f}_{3}\right\|_{* *} \leqslant C \mu^{\frac{n}{2 q}}\left(\left\|\overline{\bar{\phi}}_{1}\right\|_{*}+\|\hat{\hat{\phi}}\|_{*}+k^{1-\frac{n}{q}}+h^{1-\frac{n}{q}}\right) .
\end{aligned}
$$

Denote

Notice that

$$
f_{4}:=\bar{\zeta}_{1} N(\phi), \quad f_{5}:=\bar{\zeta}_{1} E
$$

$$
\tilde{N}(\phi)=\left|V_{*}+\tilde{\phi}_{1}\right|^{p-1}\left(V_{*}+\tilde{\phi}_{1}\right)-\left|V_{*}\right|^{p-1} V_{*}-p\left|V_{*}\right|^{p-1} \tilde{\phi}_{1},
$$


where $\tilde{\phi}_{1}(y):=\mu^{\frac{n-2}{2}} \phi\left(\xi_{1}+\mu y\right)$, and

$$
\begin{aligned}
V_{*}(y):= & -U(y)-\sum_{j=2}^{k} U\left(y+\mu^{-1}\left(\xi_{1}-\xi_{j}\right)\right) \\
& -\sum_{l=1}^{h} \mu^{\frac{n-2}{2}} \lambda^{-\frac{n-2}{2}} U\left(\lambda^{-1}\left(\xi_{1}+\mu y-\eta_{l}\right)\right) \\
& +\mu^{\frac{n-2}{2}} U\left(\xi_{1}+\mu y\right) .
\end{aligned}
$$

Hence, for

$$
\phi=\bar{\phi}_{1}+\sum_{j=2}^{k} \bar{\phi}_{j}+\sum_{l=1}^{h} \hat{\phi}_{l}+\Psi\left(\overline{\bar{\phi}}_{1}, \hat{\hat{\phi}}_{1}\right)
$$

one has

$$
\left|\tilde{\phi}_{1}\right| \leqslant C \mu^{\frac{n-2}{2}}\left(\left\|\overline{\bar{\phi}}_{1}\right\|_{*}+\left\|\hat{\hat{\phi}}_{1}\right\|_{*}\right)+\mu^{\frac{n-2}{2}}\left\|\Psi\left(\overline{\bar{\phi}}_{1}, \hat{\hat{\phi}}_{1}\right)\right\|_{L^{\infty}\left(\mathbb{R}^{n}\right)} .
$$

Furthermore, in the region $|y|<\frac{\bar{\alpha}}{\mu k}$ it holds $U(y) \sim \mu^{\frac{n-2}{2}}$ and thus, after a second order Taylor expansion one has

$$
\begin{aligned}
\left|\tilde{f}_{4}(y)\right| & \leqslant C\left|V_{*}\right|^{p-2}\left|\tilde{\phi}_{1}\right|^{2} \leqslant C U^{p-2} \mu^{\frac{n-2}{2}}\left|\tilde{\phi}_{1}\right| \\
& \leqslant C U^{p-1} \mu^{\frac{n-2}{2}}\left(\left\|\overline{\bar{\phi}}_{1}\right\|_{*}+\left\|\hat{\hat{\phi}}_{1}\right\|_{*}+\|\Psi\|_{*}\right),
\end{aligned}
$$

and

$$
\left\|\tilde{f}_{4}\right\|_{* *} \leqslant C \mu^{\frac{n}{2 q}}\left(\left\|\overline{\bar{\phi}}_{1}\right\|_{*}+\left\|\hat{\hat{\phi}}_{1}\right\|_{*}+k^{1-\frac{n}{q}}+h^{1-\frac{n}{q}}\right) .
$$

Finally, by (1.1.6), we know

$$
\left\|\tilde{f}_{5}\right\|_{* *} \leqslant C \mu^{\frac{n}{2 q}}
$$

Likewise, one can obtain analogous estimates for

$$
\hat{T}\left(\hat{\zeta}_{1} E+\gamma \hat{\mathcal{N}}\left(\overline{\bar{\phi}}_{1}, \hat{\hat{\phi}}_{1}\right)\right)
$$

to conclude that $\mathcal{M}$ maps functions $\phi_{1}$ with $\left\|\phi_{1}\right\|_{*} \leqslant C\left(\mu^{\frac{n}{2 q}}+\lambda^{\frac{n}{2 q}}\right)$ into the same class of functions. Besides, one can prove that the map is indeed a contraction, and thus we conclude the existence of a unique solution to the system (1.2.32).

Remark 1.2.5. The symmetry conditions (2.2.17) and (2.2.19) follow straightforward as consequence of the uniqueness.

\subsection{Proof of Theorem 1.0 .1}

Thanks to Proposition 1.2.4 we have $\bar{\phi}_{1}$ and $\hat{\phi}_{1}$ solutions to (1.2.32). Thus, if we find $\delta$ and $\varepsilon$ in (0.0.7) so that $\bar{c}_{0}(\delta, \varepsilon)=\hat{c}_{0}(\delta, \varepsilon)=0$ they actually solve (1.2.25) and (1.2.30). Repeating this argument for every $j=1, \ldots, k-1$ and $l=1, \ldots, h-1$ we conclude that

$$
u=U_{*}+\phi
$$


with $\phi$ defined in (1.2.1) is the solution to problem (0.0.1) we were looking for. Thus, we want to prove the existence of $\delta$ and $\varepsilon$ so that (we keep the names in an abuse of notation)

$$
\begin{aligned}
& \bar{c}_{0}(\delta, \varepsilon)=\int_{\mathbb{R}^{n}}\left(\bar{\zeta}_{1} E+\gamma \overline{\mathcal{N}}\left(\overline{\bar{\phi}}_{1}, \hat{\hat{\phi}}_{1}\right)\right) \bar{Z}_{0}=0, \\
& \hat{c}_{0}(\delta, \varepsilon)=\int_{\mathbb{R}^{n}}\left(\hat{\zeta}_{1} E+\gamma \hat{\mathcal{N}}\left(\overline{\bar{\phi}}_{1}, \hat{\hat{\phi}}_{1}\right)\right) \hat{Z}_{0}=0 .
\end{aligned}
$$

Indeed, we will prove that

$$
\begin{aligned}
& \bar{c}_{0}(\delta, \varepsilon)=-A_{n} \frac{\delta}{k^{n-2}}\left[\delta a_{n, k}^{1}-a_{n, k}^{2}\right]+\frac{1}{k^{n-1}} \Theta_{k, h}(\delta, \varepsilon), \\
& \hat{c}_{0}(\delta, \varepsilon)=-A_{n} \frac{\varepsilon}{h^{n-2}}\left[\varepsilon b_{n, h}^{1}-b_{n, h}^{2}\right]+\frac{1}{h^{n-1}} \Theta_{k, h}(\delta, \varepsilon) .
\end{aligned}
$$

Here $A_{n}$ is a fixed positive constant that depends on $n$, while for $i=1,2, a_{n, k}^{i}, b_{n, h}^{i}$ are positive constants, of the form $a_{n, k}^{i}=a_{n}^{i}+O\left(\frac{1}{k}\right), b_{n, h}^{i}=b_{n}^{i}+O\left(\frac{1}{h}\right)$, as $k, h \rightarrow \infty$, with $a_{n}^{i}$ and $b_{n}^{i}$ positive constants. Furthermore, $\Theta_{k, h}(\delta, \varepsilon)$ denotes a generic function, which is smooth in its variables, and it is uniformly bounded, together with its first derivatives, in $\delta$ and $\varepsilon$ satisfying the bounds (0.0.7), when $k \rightarrow \infty$ and $h \rightarrow \infty$. By a fixed point argument one can prove the existence of a solution $(\delta, \varepsilon)$ to the system

$$
\bar{c}_{0}(\delta, \varepsilon)=\hat{c}_{0}(\delta, \varepsilon)=0 .
$$

Thus, if we prove that (1.3.1) holds, we conclude the proof of Theorem 1.0.1

Both estimates in (1.3.1) follow in the same way, so let us prove the first one. We write

$$
\bar{c}_{0}(\delta, \varepsilon)=\int_{\mathbb{R}^{n}} E \bar{Z}_{0}+\int_{\mathbb{R}^{n}}\left(\bar{\zeta}_{1}-1\right) E \bar{Z}_{0}+\gamma \int_{\mathbb{R}^{n}} \overline{\mathcal{N}}\left(\overline{\bar{\phi}}_{1}, \hat{\hat{\phi}}_{1}\right) \bar{Z}_{0}
$$

and we analyze every term independently. For $\delta$ and $\varepsilon$ satisfying (0.0.7), we have that

\section{Claim 1:}

Claim 2:

$$
\int_{\mathbb{R}^{n}} E \bar{Z}_{0}=-A_{n} \frac{\delta}{k^{n-2}}\left[\delta a_{n, k}^{1}-a_{n, k}^{2}\right]+\frac{1}{k^{n-1}} \Theta_{k, h}(\delta, \varepsilon)
$$

$$
\int_{\mathbb{R}^{n}}\left(\bar{\zeta}_{1}-1\right) E \bar{Z}_{0}=\frac{1}{k^{n-1}} \Theta_{k, h}(\delta, \varepsilon)
$$

Claim 3:

$$
\int_{\mathbb{R}^{n}} \overline{\mathcal{N}}\left(\overline{\bar{\phi}}_{1}, \hat{\hat{\phi}}_{1}\right) \bar{Z}_{0}=\frac{1}{k^{n-1}} \Theta_{k, h}(\delta, \varepsilon),
$$

as $k$ and $h \rightarrow \infty$. It is clear that these claims imply the validity of the first equation in (1.3.1).

Proof of Claim 1. Let us denote

$$
\text { Ext }:=\left\{\cap_{j=1}^{k}\left\{\left|y-\xi_{j}\right|>\frac{\bar{\alpha}}{k}\right\}\right\} \cap\left\{\cap_{l=1}^{h}\left\{\left|y-\eta_{l}\right|>\frac{\hat{\alpha}}{h}\right\}\right\} .
$$

For $\bar{\alpha}>0$ independent of $k$ we can write the first term as

$$
\int_{\mathbb{R}^{n}} E \bar{Z}_{0}=\int_{B\left(\xi_{1}, \frac{\bar{\alpha}}{k}\right)} E \bar{Z}_{0}+\int_{E x t} E \bar{Z}_{0}+\sum_{j \neq 1} \int_{B\left(\xi_{j}, \frac{\bar{\alpha}}{k}\right)} E \bar{Z}_{0}+\sum_{l=1}^{h} \int_{B\left(\eta_{l}, \frac{\hat{\alpha}}{h}\right)} E \bar{Z}_{0} .
$$


Considering $\bar{E}_{1}(y)=\mu^{\frac{n+2}{2}} E\left(\xi_{1}+\mu y\right)$ and using (1.1.4) we obtain

$$
\begin{aligned}
\int_{B\left(\xi_{1}, \frac{\bar{\alpha}}{k}\right)} E \bar{Z}_{0}= & \int_{B\left(0, \frac{\bar{\alpha}}{\mu k}\right)} \bar{E}_{1}(y) Z_{0}(y) d y \\
= & -\gamma p \sum_{j \neq 1} \int_{B\left(0, \frac{\bar{\alpha}}{\mu k}\right)} U^{p-1} U\left(y-\mu^{-1}\left(\xi_{j}-\xi_{1}\right)\right) Z_{0} d y \\
& +\gamma p \mu^{\frac{n-2}{2}} \int_{B\left(0, \frac{\bar{\alpha}}{\mu k}\right)} U^{p-1} U\left(\xi_{1}+\mu y\right) Z_{0} d y \\
& -\gamma p \sum_{l=1}^{h} \mu^{\frac{n-2}{2}} \lambda^{-\frac{n-2}{2}} \int_{B\left(0, \frac{\bar{\alpha}}{\mu k}\right)} U^{p-1} U\left(\lambda^{-1}\left(\xi_{1}+\mu y-\eta_{l}\right)\right) Z_{0} d y \\
& +\gamma p \int_{B\left(0, \frac{\bar{\alpha}}{\mu k}\right)}\left[(U(y)+s V)^{p-1}-U^{p-1}\right] V(y) Z_{0} d y \\
& -\mu^{\frac{n+2}{2}} \int_{B\left(0, \frac{\bar{\alpha}}{\mu k}\right)} U^{p}\left(\xi_{1}+\mu y\right) Z_{0} d y \\
& -\sum_{j \neq 1} \int_{B\left(0, \frac{\bar{\alpha}}{\mu k}\right)} U^{p}\left(y-\mu^{-1}\left(\xi_{j}-\xi_{1}\right)\right) Z_{0} d y \\
& -\sum_{l=1}^{h} \mu^{\frac{n+2}{2}} \lambda^{-\frac{n+2}{2}} \int_{B\left(0, \frac{\bar{\alpha}}{\mu k}\right)} U^{p}\left(\lambda^{-1}\left(\xi_{1}+\mu y-\eta_{l}\right)\right) Z_{0} d y
\end{aligned}
$$

where

$$
\begin{aligned}
V(y):= & -\sum_{j \neq 1} U\left(y-\mu^{-1}\left(\xi_{j}-\xi_{1}\right)\right)+\mu^{\frac{n-2}{2}} U\left(\xi_{1}+\mu y\right) \\
& -\sum_{l=1}^{h} \mu^{\frac{n-2}{2}} \lambda^{-\frac{n-2}{2}} U\left(\lambda^{-1}\left(\xi_{1}+\mu y-\eta_{l}\right)\right) .
\end{aligned}
$$

Doing a Taylor expansion, for $j \neq 1$ there holds

$$
\int_{B\left(0, \frac{\bar{\alpha}}{\mu k}\right)} U^{p-1} U\left(y-\mu^{-1}\left(\xi_{j}-\xi_{1}\right)\right) Z_{0} d y=\frac{c_{1} \mu^{n-2}}{\left|\xi_{j}-\xi_{1}\right|^{n-2}}\left(1+\frac{\mu^{2}}{\left|\xi_{j}-\xi_{1}\right|^{2}} \Theta_{k, h}(\delta, \varepsilon)\right),
$$

and

$$
\int_{B\left(0, \frac{\bar{\alpha}}{\mu k}\right)} U^{p-1} U\left(\lambda^{-1}\left(\xi_{1}+\mu y-\eta_{l}\right)\right) Z_{0} d y=\frac{c_{1} \lambda^{n-2}}{\left|\xi_{1}-\eta_{l}\right|^{n-2}}\left(1+\frac{\lambda^{2}}{\left|\xi_{j}-\xi_{1}\right|^{2}} \Theta_{k, h}(\delta, \varepsilon)\right),
$$

where $c_{1}$ is some positive constant, and, as before, $\Theta_{k, h}(\delta, \varepsilon)$ denotes a generic function, which is smooth in its variables, and it is uniformly bounded, together with its first derivatives, in $\delta$ and $\varepsilon$ satisfying the bounds (0.0.7), when $k \rightarrow \infty$ and $h \rightarrow \infty$. Proceeding in a similar way,

$$
\mu^{\frac{n-2}{2}} \int_{B\left(0, \frac{\bar{\alpha}}{\mu k}\right)} U^{p-1} U\left(\xi_{1}+\mu y\right) Z_{0} d y=c_{2} \mu^{\frac{n-2}{2}}\left(1+(\mu k)^{2} \Theta_{k, h}(\delta, \varepsilon)\right),
$$


for some positive constant $c_{2}$. On the other hand,

$$
\begin{aligned}
\left|\mu^{\frac{n+2}{2}} \int_{B\left(0, \frac{\bar{\alpha}}{\mu k}\right)} U^{p}\left(\xi_{1}+\mu y\right) Z_{0} d y\right| & \leqslant C \mu^{\frac{n+2}{2}} \int_{B\left(0, \frac{\bar{\alpha}}{\mu k}\right)} \frac{1}{(1+|y|)^{n-2}} \leqslant C \mu^{\frac{n-2}{2}} k^{-2}, \\
\left|\sum_{j \neq 1} \int_{B\left(0, \frac{\bar{\alpha}}{\mu k}\right)} U^{p}\left(y-\mu^{-1}\left(\xi_{j}-\xi_{1}\right)\right) Z_{0} d y\right| & \leqslant \sum_{j \neq 1} \frac{\mu^{n+2}}{\left|\xi_{j}-\xi_{1}\right|^{n+2}} \int_{B\left(0, \frac{\bar{\alpha}}{\mu k}\right)} \frac{1}{(1+|y|)^{n-2}} \\
& \leqslant C(\mu k)^{-2} \sum_{j \neq 1} \frac{\mu^{n+2}}{\left|\xi_{j}-\xi_{1}\right|^{n+2}},
\end{aligned}
$$

and

$$
\begin{aligned}
& \left|\sum_{l=1}^{h} \mu^{\frac{n-2}{2}} \lambda^{-\frac{n+2}{2}} \int_{B\left(0, \frac{\bar{\alpha}}{\mu k}\right)} U^{p}\left(\lambda^{-1}\left(\xi_{1}+\mu y-\eta_{l}\right)\right) Z_{0}\right| \\
& \leqslant C \sum_{l=1}^{h} \mu^{\frac{n-2}{2}} \lambda^{\frac{n+2}{2}} \int_{B\left(0, \frac{\bar{\alpha}}{\mu k}\right)} \frac{1}{(1+|y|)^{n-2}} \\
& \leqslant C \mu^{\frac{n+2}{2}} \lambda^{\frac{n+2}{2}} h k^{2} .
\end{aligned}
$$

Finally, putting together (1.3.8), (1.3.9) and (1.3.10),

$$
\begin{aligned}
& \left|\int_{B\left(0, \frac{\bar{\alpha}}{\mu k}\right)}\left[(U(y)+s V)^{p-1}-U^{p-1}\right] V(y) Z_{0} d y\right| \\
& \leqslant C\left(\mu^{\frac{n-2}{2}} k^{-2}+(\mu k)^{-2} \sum_{j \neq 1} \frac{\mu^{n+2}}{\left|\xi_{j}-\xi_{1}\right|^{n+2}}+\mu^{\frac{n+2}{2}} \lambda^{\frac{n+2}{2}} h k^{2}\right) .
\end{aligned}
$$

To estimate the second term in (1.3.7) we apply Hölder inequality to get

$$
\left|\int_{E x t} E \bar{Z}_{0}\right| \leqslant C\left\|(1+|y|)^{n+2-\frac{2 n}{q}} E\right\|_{L^{q}(E x t)}\left\|(1+|y|)^{-n-2+\frac{2 n}{q}} \bar{Z}_{0}\right\|_{L^{\frac{q}{q-1}}(E x t)} .
$$

Proceeding as in [4] we have

$$
\left\|(1+|y|)^{-n-2+\frac{2 n}{q}} \bar{Z}_{0}\right\|_{L^{\frac{q}{q-1}}(E x t)} \leqslant C \mu^{\frac{n-2}{2}} k^{n-2} k^{\frac{n}{q}-n}
$$

and using the estimates obtained in Section 1.1 we see that

$$
\left\|(1+|y|)^{n+2-\frac{2 n}{q}} E\right\|_{L^{q}(E x t)} \leqslant C\left(\mu^{\frac{n-2}{2}} k^{n-2} k^{1-\frac{n}{q}}+\lambda^{\frac{n-2}{2}} h^{n-2} h^{1-\frac{n}{q}}\right),
$$

and thus, substituting in (1.3.12),

$$
\left|\int_{E x t} E \bar{Z}_{0}\right| \leqslant C\left(\frac{\mu^{n-2} k^{2(n-2)}}{k^{n-1}}+\frac{\mu^{\frac{n-2}{2}} \lambda^{\frac{n-2}{2}} k^{n-2} h^{n-2}}{k^{n-\frac{n}{q}} h^{\frac{n}{q}-1}}\right) .
$$


Arguing as in [4] the third term in (1.3.7) can be estimated as

$$
\left|\sum_{j \neq 1} \int_{B\left(\xi_{j}, \frac{\bar{\alpha}}{k}\right)} E \bar{Z}_{0}\right| \leqslant \frac{\mu^{\frac{n-2}{2}}}{(\mu k)^{n-4}}\left[\mu^{n-2} \sum_{j \neq 1} \frac{1}{\left|\xi_{j}-\xi_{1}\right|^{n-2}}\right] \text {. }
$$

Likewise,

$$
\begin{aligned}
\left|\int_{B\left(\eta_{l}, \frac{\hat{\alpha}}{h}\right)} E \bar{Z}_{0}\right|=\left|\lambda^{\frac{n-2}{2}} \int_{B\left(0, \frac{\hat{\alpha}}{\lambda h}\right)} \hat{E}_{l}(y) \bar{Z}_{0}\left(\lambda y+\eta_{l}\right)\right| \\
\leqslant \lambda^{\frac{n-2}{2}}\left\|(1+|y|)^{n+2-\frac{2 n}{q}} \hat{E}_{l}\right\|_{L^{q}\left(|y|<\frac{\hat{\alpha}}{\lambda h}\right)} \\
\cdot \|(1+|y|)^{-n-2+\frac{2 n}{q}} \mu^{-\frac{n-2}{2}} Z_{0}\left(\mu^{-1}\left(\lambda y+\eta_{l}-\xi_{1}\right) \|_{L^{\frac{q}{q-1}}\left(|y|<\frac{\hat{\alpha}}{\lambda h}\right)}\right.
\end{aligned}
$$

Noticing that

$$
\begin{aligned}
& \left\|(1+|y|)^{-n-2+\frac{2 n}{q}} \mu^{-\frac{n-2}{2}} Z_{0}\left(\mu^{-1}\left(\lambda y+\eta_{l}-\xi_{1}\right)\right)\right\|_{L^{\frac{q}{q-1}}\left(\mathbb{R}^{n}\right)} \\
& \leqslant C \mu^{\frac{n-2}{2}}\left(\int_{1}^{\frac{\hat{\alpha}}{\lambda h}} \frac{t^{n-1} d t}{t^{\left(n+2-\frac{2 n}{q}\right) \frac{q}{q-1}}}\right)^{\frac{q-1}{q}} \leqslant C \mu^{\frac{n-2}{2}}(\lambda h)^{2-\frac{n}{q}},
\end{aligned}
$$

by (1.1.8) we conclude that

$$
\left|\sum_{l=1}^{h} \int_{B\left(\eta_{l}, \frac{\hat{\alpha}}{h}\right)} E \bar{Z}_{0}\right| \leqslant C \lambda^{\frac{n-2}{2}} h^{-\frac{n}{q}} \mu^{\frac{n-2}{2}}(\lambda h)^{2-\frac{n}{q}} h
$$

Claim 1 follows from these estimates applying the fact that $h=O(k)$.

Proof of Claim 2. Let us estimate the second term of (1.3.3). Notice first that

$$
\left|\int_{\mathbb{R}^{n}}\left(\bar{\zeta}_{1}-1\right) E \bar{Z}_{0}\right| \leqslant C\left|\int_{\left\{\left|y-\xi_{1}\right|>\frac{\bar{\alpha}}{k}\right\}} E \bar{Z}_{0}\right|
$$

and we separate this integral as

$$
\int_{\left\{\left|y-\xi_{1}\right|>\frac{\bar{\alpha}}{k}\right\}} E \bar{Z}_{0}=\int_{E x t} E \bar{Z}_{0}+\sum_{j=2}^{k} \int_{\left\{\left|y-\xi_{j}\right| \leqslant \frac{\bar{\alpha}}{k}\right\}} E \bar{Z}_{0}+\sum_{l=1}^{h} \int_{\left\{\left|y-\eta_{l}\right| \leqslant \frac{\hat{\alpha}}{h}\right\}} E \bar{Z}_{0} .
$$

Thus, Claim 2 follows from (1.3.13), (1.3.14) and (1.3.15).

Proof of Claim 3. Notice that

$$
\int_{\mathbb{R}^{n}} \overline{\mathcal{N}}\left(\overline{\bar{\phi}}_{1}, \hat{\hat{\phi}}_{1}\right) \bar{Z}_{0}=\mu^{\frac{n+2}{2}} \int_{\mathbb{R}^{n}} \overline{\mathcal{N}}\left(\overline{\bar{\phi}}_{1}, \hat{\hat{\phi}}_{1}\right)\left(\xi_{1}+\mu y\right) Z_{0}(y),
$$

and thus, from estimates (1.2.36) - (1.2.39) and the fact $h=O(k)$ we conclude

$$
\int_{\mathbb{R}^{n}} \overline{\mathcal{N}}\left(\overline{\bar{\phi}}_{1}, \hat{\hat{\phi}}_{1}\right) \bar{Z}_{0} \leqslant C k^{3-n-\frac{n}{q}} \int_{\mathbb{R}^{n}} U^{p-1}\left|Z_{0}\right|,
$$

and the claim follows. 


\section{Part 2. Nondegeneracy}

As stated before, the goal of this part is to prove the nondegeneracy (see Definition 0.0.1) of the solution $u$ provided by Theorem 1.0.1 (we drop the dependence on $k$ and $h$ by simplicity). Recalling the functions $z_{\alpha}$ defined in (0.0.12) $-(0.0 .17)$ we can formulate the result in Theorem 0.0 .3 as follows.

Theorem 2.0.1. There exists a sequence of solutions $u$ to Problem (0.0.1) among the ones constructed in Theorem 1.0.1 for which all bounded solutions to the equation

$$
-\Delta \varphi-\gamma p|u|^{p-2} u \varphi=0
$$

are linear combination of the functions $z_{\alpha}$ for $\alpha=0, \ldots, N_{0}-1$. (Recall that $N_{0}:=5(n-1)$.)

For later simplification, we introduce the following functions

$$
\begin{aligned}
\mathbf{z}_{\beta}:=z_{\beta}, & \text { if } \quad \beta \neq n+3, n+4, n+5, n+6, \\
\mathbf{z}_{n+2+\alpha}:=\frac{z_{\alpha}-z_{n+2+\alpha}}{2}, & \text { if } \quad \alpha=1,2,3,4 .
\end{aligned}
$$

Since $\mathbf{z}_{\beta}$ are linear combinations of the original functions $z_{\beta}$, the statement of Theorem 2.0.1 is equivalent to say that there exists a sequence of solutions among the ones constructed in Theorem 1.0 .1 for which all bounded solutions to (2.0.1) are linear combinations of $\mathbf{z}_{\beta}$, for $\beta=0, \ldots, N_{0}-1$.

Thus, let $\varphi$ be a bounded solution of (2.0.1), namely $L(\varphi)=0$, with $L$ defined in (0.0.18). We decompose $\varphi$ as

$$
\varphi(y)=\sum_{\beta=0}^{N_{0}-1} a_{\beta} \mathbf{z}_{\beta}(y)+\tilde{\varphi}(y)
$$

where $a_{\beta}$ are chosen so that

$$
\int_{\mathbb{R}^{n}}|u|^{p-1} \mathbf{z}_{\beta} \tilde{\varphi}=0
$$

holds. Notice that, since $\mathbf{z}_{\beta} \in \operatorname{ker}\{L\}$, one has $L(\tilde{\varphi})=0$ and thus our goal will be to prove that actually $\tilde{\varphi} \equiv 0$.

Recall that our solution $u$ has the form

$$
u(y)=U(y)-\sum_{j=1}^{k} U_{\mu, \xi_{j}}(y)-\sum_{l=1}^{h} U_{\lambda, \eta_{l}}(y)+\phi(y),
$$

where $\phi$ is defined in (1.2.1) as $\phi=\sum_{j=1}^{k} \bar{\phi}_{j}+\sum_{l=1}^{h} \hat{\phi}_{l}+\psi$.

We introduce the following functions

$$
\begin{aligned}
& Z_{00}(y):=\frac{n-2}{2}[U+\psi](y)+\nabla[U+\psi](y) \cdot y, \\
& Z_{\alpha 0}(y):=\frac{\partial}{\partial y_{\alpha}} U(y)+\frac{\partial}{\partial y_{\alpha}} \psi(y) \quad \alpha=1, \ldots, n .
\end{aligned}
$$


For $j$ fixed in $\{1, \ldots, k\}$, we define

$$
\begin{aligned}
& \bar{Z}_{0 j}(y):=\frac{n-2}{2}\left[U_{\mu, \xi_{j}}+\bar{\phi}_{j}\right](y)+\nabla\left[U_{\mu, \xi_{j}}+\bar{\phi}_{j}\right](y) \cdot\left(y-\xi_{j}\right) \\
& \bar{Z}_{1 j}(y):=\xi_{j} \cdot \nabla_{y}\left[U_{\mu, \xi_{j}}+\bar{\phi}_{j}\right](y), \quad \bar{Z}_{2 j}(y):=\xi_{j}^{\perp} \cdot \nabla_{y}\left[U_{\mu, \xi_{j}}+\bar{\phi}_{j}\right](y), \\
& \bar{Z}_{\alpha j}(y):=\frac{\partial}{\partial y_{\alpha}}\left[U_{\mu, \xi_{j}}(y)+\bar{\phi}_{j}\right], \quad \alpha=3, \ldots, n .
\end{aligned}
$$

For $l$ fixed in $\{1, \ldots, h\}$, we define

$$
\begin{aligned}
& \hat{Z}_{0 j}(y):=\frac{n-2}{2}\left[U_{\lambda, \eta_{l}}+\hat{\phi}_{l}\right](y)+\nabla\left[U_{\lambda, \eta_{l}}+\hat{\phi}_{l}\right](y) \cdot\left(y-\eta_{l}\right) \\
& \hat{Z}_{3 l}(y):=\eta_{l} \cdot \nabla_{y}\left[U_{\lambda, \eta_{l}}+\hat{\phi}_{l}\right](y), \quad \hat{Z}_{4 l}(y):=\eta_{l}^{\perp} \cdot \nabla_{y}\left[U_{\lambda, \eta_{l}}+\hat{\phi}_{l}\right](y), \\
& \hat{Z}_{\alpha l}(y):=\frac{\partial}{\partial y_{\alpha}}\left[U_{\lambda, \eta_{l}}(y)+\hat{\phi}_{l}\right], \quad \alpha=1,2,5,6, \ldots, n .
\end{aligned}
$$

In Appendix 2.6 we provide the expressions of the functions $\mathbf{z}_{\beta}, \beta=0, \ldots, N_{0}-1$, in terms of the functions $Z_{\alpha 0}, \bar{Z}_{\alpha j}, j=1, \ldots, k$, and $\hat{Z}_{\alpha, l}, l=1, \ldots, h$, for any $\alpha=0, \ldots, n$. These relations will be useful in other parts of our argument.

We rearrange the functions above in $(n+1)$ vector fields as

$$
\Pi_{\alpha}:=\left[Z_{\alpha 0}, \bar{Z}_{\alpha 1}, \ldots, \bar{Z}_{\alpha k}, \hat{Z}_{\alpha 1}, \ldots, \hat{Z}_{\alpha h}\right]^{T}, \quad \alpha=0,1, \ldots, n,
$$

and, for any given vector $d=\left[d_{0}, \bar{d}_{1}, \ldots, \bar{d}_{k}, \hat{d}_{1}, \ldots, \hat{d}_{h}\right]^{T} \in \mathbb{R}^{1+k+h}$ we use the notation

$$
d \cdot \Pi_{\alpha}:=d_{0} Z_{\alpha 0}+\sum_{j=1}^{k} \bar{d}_{j} \bar{Z}_{\alpha j}+\sum_{l=1}^{h} \hat{d}_{l} \hat{Z}_{\alpha l} .
$$

With this in mind, we write the function $\tilde{\varphi}$ in (2.0.3) as

$$
\tilde{\varphi}(y)=\sum_{\alpha=0}^{n} c_{\alpha} \cdot \Pi_{\alpha}(y)+\varphi^{\perp}(y)
$$

where

$$
c_{\alpha}:=\left[c_{\alpha 0}, \bar{c}_{\alpha 1}, \ldots, \bar{c}_{\alpha k}, \hat{c}_{\alpha 1}, \ldots, \hat{c}_{\alpha h}\right]^{T}, \quad \alpha=0, \ldots, n,
$$

are $(n+1)$ vectors in $\mathbb{R}^{k+h+1}$ chosen so that

$$
\begin{aligned}
& \int_{\mathbb{R}^{n}} U^{p-1} Z_{\alpha 0} \varphi^{\perp}=0, \quad \alpha=0,1, \ldots, n, \\
& \int_{\mathbb{R}^{n}} U_{\mu, \xi_{j}}^{p-1} \bar{Z}_{\alpha j} \varphi^{\perp}=0, \quad j=1, \ldots, k, \alpha=0,1, \ldots, n, \\
& \int_{\mathbb{R}^{n}} U_{\lambda, \eta_{l}}^{p-1} \hat{Z}_{\alpha l} \varphi^{\perp}=0, \quad l=1, \ldots, h, \quad \alpha=0,1, \ldots, n .
\end{aligned}
$$

Hence, to prove that $\tilde{\varphi} \equiv 0$ we have to see that $c_{\alpha}=0$ for every $\alpha$ and $\varphi^{\perp} \equiv 0$. This will be consequence of the following three facts. 
Fact 1: Since $L(\tilde{\varphi})=0$, one has that

$$
\sum_{\alpha=0}^{n} c_{\alpha} \cdot L\left(\Pi_{\alpha}\right)=-L\left(\varphi^{\perp}\right),
$$

with $L$ defined in (0.0.18). We write $\varphi^{\perp}=\varphi_{0}^{\perp}+\sum_{j=1}^{k} \bar{\varphi}_{j}^{\perp}+\sum_{l=1}^{h} \hat{\varphi}_{l}^{\perp}$, where

$$
\begin{aligned}
& -L\left(\varphi_{0}^{\perp}\right)=\sum_{\alpha=0}^{n} c_{\alpha 0} L\left(Z_{\alpha 0}\right), \\
& -L\left(\bar{\varphi}_{j}^{\perp}\right)=\sum_{\alpha=0}^{n} \bar{c}_{\alpha j} L\left(\bar{Z}_{\alpha j}\right), \quad j=1, \ldots, k, \\
& -L\left(\hat{\varphi}_{l}^{\perp}\right)=\sum_{\alpha=0}^{n} \hat{c}_{\alpha l} L\left(\hat{Z}_{\alpha l}\right), \quad l=1, \ldots, h .
\end{aligned}
$$

Furthermore, let us define

$$
\overline{\bar{\varphi}}_{j}^{\perp}(y):=\mu^{\frac{n-2}{2}} \bar{\varphi}_{j}^{\perp}\left(\mu y+\xi_{j}\right), \quad \hat{\hat{\varphi}}_{l}^{\perp}(y):=\lambda^{\frac{n-2}{2}} \hat{\varphi}_{l}^{\perp}\left(\lambda y+\eta_{l}\right),
$$

and

$$
\left\|\varphi^{\perp}\right\|:=\left\|\varphi_{0}^{\perp}\right\|_{*}+\sum_{j=1}^{k}\left\|\overline{\bar{\varphi}}_{j}^{\perp}\right\|_{*}+\sum_{l=1}^{h}\left\|\hat{\hat{\varphi}}_{l}^{\perp}\right\|_{*} .
$$

Thus, as we will prove in Section 2.4, there exists a positive constant $C$ such that

$$
\left\|\varphi^{\perp}\right\| \leqslant C k^{-2+\frac{2}{n}} \sum_{\alpha=0}^{n}\left\|c_{\alpha}\right\| .
$$

Fact 2: Condition (2.0.4) is equivalent to

$$
\begin{aligned}
\sum_{\alpha=0}^{n} c_{\alpha} \cdot \int_{\mathbb{R}^{n}} \Pi_{\alpha}|u|^{p-1} \mathbf{z}_{\beta}= & \sum_{\alpha=0}^{n}\left[c_{\alpha 0} \int_{\mathbb{R}^{n}} Z_{\alpha 0}|u|^{p-1} \mathbf{z}_{\beta}+\sum_{j=1}^{k} \bar{c}_{\alpha j} \int_{\mathbb{R}^{n}} \bar{Z}_{\alpha j}|u|^{p-1} \mathbf{z}_{\beta}\right. \\
& \left.+\sum_{l=1}^{h} \hat{c}_{\alpha l} \int_{\mathbb{R}^{n}} \hat{Z}_{\alpha l}|u|^{p-1} \mathbf{z}_{\beta}\right] \\
= & -\int_{\mathbb{R}^{n}} \varphi^{\perp}|u|^{p-1} \mathbf{z}_{\beta}, \quad \beta=0, \ldots, N_{0}-1 .
\end{aligned}
$$

Let us denote

$$
\begin{aligned}
\overline{\cos }:= & {\left[\begin{array}{c}
1 \\
\cos \bar{\theta}_{2} \\
\ldots \\
\cos \bar{\theta}_{k-1}
\end{array}\right], \overline{\sin }:=\left[\begin{array}{c}
0 \\
\sin \bar{\theta}_{2} \\
\ldots \\
\sin \bar{\theta}_{k-1}
\end{array}\right], \quad \bar{\theta}_{j}:=\frac{2 \pi}{k}(j-1), } \\
\operatorname{côs} & :=\left[\begin{array}{c}
1 \\
\cos \hat{\theta}_{2} \\
\ldots \\
\cos \hat{\theta}_{h-1}
\end{array}\right], \hat{\sin }:=\left[\begin{array}{c}
0 \\
\sin \hat{\theta}_{2} \\
\ldots \\
\sin \hat{\theta}_{h-1}
\end{array}\right], \quad \hat{\theta}_{l}:=\frac{2 \pi}{h}(l-1),
\end{aligned}
$$


and

$$
\overline{1}:=\left[\begin{array}{c}
1 \\
1 \\
\ldots \\
1
\end{array}\right], \quad \hat{1}:=\left[\begin{array}{c}
1 \\
1 \\
\ldots \\
1
\end{array}\right], \quad \overline{0}:=\left[\begin{array}{c}
0 \\
0 \\
\ldots \\
0
\end{array}\right], \quad \hat{0}:=\left[\begin{array}{c}
0 \\
0 \\
\ldots \\
0
\end{array}\right]
$$

where $\overline{1}$ and $\overline{0}$ are $k$-dimensional vectors, and $\hat{1}$ and $\hat{0}$ are vectors of dimension $h$. Likewise, define

$$
\tilde{c}_{0}:=\left[\begin{array}{c}
c_{00} \\
\cdots \\
c_{n 0}
\end{array}\right] \in \mathbb{R}^{n+1}, \quad \bar{c}_{\alpha}:=\left[\begin{array}{c}
\bar{c}_{\alpha 1} \\
\ldots \\
\bar{c}_{\alpha k}
\end{array}\right] \in \mathbb{R}^{k}, \quad \hat{c}_{\alpha}:=\left[\begin{array}{c}
\hat{c}_{\alpha 1} \\
\ldots \\
\hat{c}_{\alpha h}
\end{array}\right] \in \mathbb{R}^{h}
$$

and

$$
\bar{c}:=\left[\begin{array}{c}
\bar{c}_{0} \\
\bar{c}_{1} \\
\cdots \\
\bar{c}_{n+1}
\end{array}\right] \in \mathbb{R}^{(n+1) k}, \quad \hat{c}:=\left[\begin{array}{c}
\hat{c}_{0} \\
\hat{c}_{1} \\
\cdots \\
\hat{c}_{n}
\end{array}\right] \in \mathbb{R}^{(n+1) h} .
$$

Thus,

Proposition 2.0.2. Solving system (2.0.11) is equivalent to solve

$$
\begin{aligned}
& c_{0} \cdot\left[\begin{array}{c}
1 \\
-\overline{1} \\
\hat{1}
\end{array}\right]+c_{1} \cdot\left[\begin{array}{c}
0 \\
-\overline{1} \\
\hat{0}
\end{array}\right]+c_{3} \cdot\left[\begin{array}{c}
0 \\
\overline{0} \\
-\hat{1}
\end{array}\right]=t_{0} \\
& +R_{h, k}\left[c_{0}, c_{1}, \ldots c_{n}\right], \\
& c_{1} \cdot\left[\begin{array}{c}
1 \\
-\overline{\cos } \\
-\hat{1}
\end{array}\right]+c_{2} \cdot\left[\begin{array}{c}
\frac{0}{\sin } \\
\hat{0}
\end{array}\right]=t_{1}+R_{h, k}\left[c_{0}, c_{1}, \ldots c_{n}\right], \\
& c_{1} \cdot\left[\begin{array}{c}
\frac{0}{-\sin } \\
\hat{0}
\end{array}\right]+c_{2} \cdot\left[\begin{array}{c}
1 \\
-\overline{\cos } \\
-\hat{1}
\end{array}\right]=t_{2}+R_{h, k}\left[c_{0}, c_{1}, \ldots c_{n}\right] \\
& c_{3} \cdot\left[\begin{array}{c}
1 \\
-\overline{1} \\
-\operatorname{côs}
\end{array}\right]+c_{4} \cdot\left[\begin{array}{c}
0 \\
\overline{0} \\
\hat{\sin }
\end{array}\right]=t_{3}+R_{h, k}\left[c_{0}, c_{1}, \ldots c_{n}\right], \\
& c_{3} \cdot\left[\begin{array}{c}
0 \\
\overline{0} \\
-\hat{\sin }
\end{array}\right]+c_{4} \cdot\left[\begin{array}{c}
1 \\
-\overline{1} \\
-\operatorname{côs}
\end{array}\right]=t_{4}+R_{h, k}\left[c_{0}, c_{1}, \ldots c_{n}\right] \\
& c_{\alpha} \cdot\left[\begin{array}{c}
1 \\
-\overline{1} \\
-\hat{1}
\end{array}\right]=t_{\alpha}+R_{h, k}\left[c_{0}, c_{1}, \ldots c_{n}\right]
\end{aligned}
$$


for $\alpha=5, \ldots, n$,

$$
\begin{aligned}
& c_{2} \cdot\left[\begin{array}{c}
0 \\
\overline{1} \\
\hat{0}
\end{array}\right]=t_{n+1}+R_{h, k}\left[c_{0}, c_{1}, \ldots c_{n}\right] \text {, } \\
& c_{4} \cdot\left[\begin{array}{c}
0 \\
\overline{0} \\
\hat{1}
\end{array}\right]=t_{n+2}+R_{h, k}\left[c_{0}, c_{1}, \ldots c_{n}\right] \text {, } \\
& c_{0} \cdot\left[\begin{array}{c}
0 \\
\overline{\cos } \\
\hat{0}
\end{array}\right]-c_{1} \cdot\left[\begin{array}{c}
0 \\
\overline{\cos } \\
\hat{0}
\end{array}\right]=t_{n+3}+R_{h, k}\left[c_{0}, c_{1}, \ldots c_{n}\right] \text {, } \\
& c_{0} \cdot\left[\begin{array}{c}
\frac{0}{\sin } \\
\hat{0}
\end{array}\right]-c_{1} \cdot\left[\begin{array}{c}
\frac{0}{\sin } \\
\hat{0}
\end{array}\right]=t_{n+4}+R_{h, k}\left[c_{0}, c_{1}, \ldots c_{n}\right] \text {, } \\
& c_{0} \cdot\left[\begin{array}{c}
0 \\
\overline{0} \\
\text { côs }
\end{array}\right]-c_{3} \cdot\left[\begin{array}{c}
0 \\
\overline{0} \\
\text { côs }
\end{array}\right]=t_{n+5}+R_{h, k}\left[c_{0}, c_{1}, \ldots c_{n}\right] \text {, } \\
& c_{0} \cdot\left[\begin{array}{c}
0 \\
\overline{0} \\
\hat{\sin }
\end{array}\right]-c_{3} \cdot\left[\begin{array}{c}
0 \\
\overline{0} \\
\hat{\sin }
\end{array}\right]=t_{n+6}+R_{h, k}\left[c_{0}, c_{1}, \ldots c_{n}\right] \text {, } \\
& c_{1} \cdot\left[\begin{array}{c}
0 \\
\overline{0} \\
\cos
\end{array}\right]+c_{3} \cdot\left[\begin{array}{c}
0 \\
-\overline{\cos } \\
\hat{0}
\end{array}\right]=t_{n+7}+R_{h, k}\left[c_{0}, c_{1}, \ldots c_{n}\right] \text {, } \\
& c_{1} \cdot\left[\begin{array}{c}
0 \\
\overline{0} \\
\hat{\sin }
\end{array}\right]+c_{4} \cdot\left[\begin{array}{c}
0 \\
-\overline{\cos } \\
\hat{0}
\end{array}\right]=t_{n+8}+R_{h, k}\left[c_{0}, c_{1}, \ldots c_{n}\right] \text {, } \\
& c_{\alpha} \cdot\left[\begin{array}{c}
0 \\
-\overline{\cos } \\
\hat{0}
\end{array}\right]=t_{n+\alpha+4}+R_{h, k}\left[c_{0}, c_{1}, \ldots c_{n}\right] \text {, }
\end{aligned}
$$

for $\alpha=5, \ldots, n$,

$$
\begin{gathered}
c_{2} \cdot\left[\begin{array}{c}
0 \\
\overline{0} \\
\operatorname{côs}
\end{array}\right]+c_{3} \cdot\left[\begin{array}{c}
\frac{0}{-\overline{\sin }} \\
\hat{0}
\end{array}\right]=t_{2 n+5}+R_{h, k}\left[c_{0}, c_{1}, \ldots c_{n}\right], \\
c_{2} \cdot\left[\begin{array}{c}
0 \\
\overline{0} \\
\hat{\sin }
\end{array}\right]+c_{4} \cdot\left[\begin{array}{c}
\frac{0}{-\sin } \\
\hat{0}
\end{array}\right]=t_{2 n+6}+R_{h, k}\left[c_{0}, c_{1}, \ldots c_{n}\right],
\end{gathered}
$$


and, for $\alpha=5, \ldots, n$,

$$
\begin{gathered}
c_{\alpha} \cdot\left[\begin{array}{c}
\frac{0}{-\overline{\sin }} \\
\hat{0}
\end{array}\right]=t_{2 n+\alpha+2}+R_{h, k}\left[c_{0}, c_{1}, \ldots c_{n}\right], \\
c_{\alpha} \cdot\left[\begin{array}{c}
0 \\
\overline{0} \\
-\hat{\sin }
\end{array}\right]=t_{3 n+\alpha-2}+R_{h, k}\left[c_{0}, c_{1}, \ldots c_{n}\right], \\
c_{\alpha} \cdot\left[\begin{array}{c}
0 \\
\overline{0} \\
-\operatorname{côs}
\end{array}\right]=t_{4 n+\alpha-6}+R_{h, k}\left[c_{0}, c_{1}, \ldots c_{n}\right] .
\end{gathered}
$$

Here $t_{i}, i=0, \ldots, 5 n-6$, are fixed numbers such that

$$
\left\|t_{i}\right\| \leqslant C\left\|\varphi^{\perp}\right\|
$$

Moreover, $R_{h, k}\left[c_{0}, c_{1}, \ldots c_{n}\right]$ stands for a function, whose specific definition changes from line to line, which can be described as follows:

$$
\begin{aligned}
R_{h, k}\left[c_{0}, c_{1}, \ldots c_{n}\right] & =\Theta_{k, h} \mathcal{L}\left(c_{00}, \ldots, c_{n 0}\right)+\bar{\Theta}_{k, h} \overline{\mathcal{L}}\left(\bar{c}_{1}, \ldots, \bar{c}_{k}\right) \\
& +\hat{\Theta}_{k, h} \hat{\mathcal{L}}\left(\hat{c}_{1}, \ldots, \hat{c}_{h}\right)
\end{aligned}
$$

and $\mathcal{L}: \mathbb{R}^{n+1} \rightarrow \mathbb{R}, \overline{\mathcal{L}}: \mathbb{R}^{k(n+1)} \rightarrow \mathbb{R}, \hat{\mathcal{L}}: \mathbb{R}^{h(n+1)} \rightarrow \mathbb{R}$ are linear functions uniformly bounded when $k, h \rightarrow \infty$, and

$$
\Theta_{k, h}=O\left(k^{1-\frac{n}{q}}\right), \quad \bar{\Theta}_{k, h}=O\left(k^{-\frac{n}{q}}\right), \quad \hat{\Theta}_{k, h}=O\left(k^{-\frac{n}{q}}\right),
$$

where $O(1)$ denotes a quantity uniformly bounded when $k, h \rightarrow \infty$, and $\frac{n}{2}<q<n$ is the number fixed in (0.0.19).

We will prove this result in Section 2.3.

Fact 3: Multiplying (2.0.9) for every $Z_{\alpha 0}, \bar{Z}_{\alpha j}, \hat{Z}_{\alpha l}, \alpha=0,1, \ldots, n, j=1, \ldots, k$ and $l=1, \ldots, h$ and integrating in $\mathbb{R}^{n}$ we get a system of the form

$$
M\left[\begin{array}{c}
c_{0} \\
c_{1} \\
\vdots \\
c_{n}
\end{array}\right]=-\left[\begin{array}{c}
r_{0} \\
r_{1} \\
\vdots \\
r_{n}
\end{array}\right] \quad \text { with } \quad r_{\alpha}:=\left[\begin{array}{c}
\int_{\mathbb{R}^{n}} L\left(\varphi^{\perp}\right) Z_{\alpha 0} \\
\int_{\mathbb{R}^{n}} L\left(\varphi^{\perp}\right) \bar{Z}_{\alpha 1} \\
\vdots \\
\int_{\mathbb{R}^{n}} L\left(\varphi^{\perp}\right) \bar{Z}_{\alpha k} \\
\int_{\mathbb{R}^{n}} L\left(\varphi^{\perp}\right) \hat{Z}_{\alpha 1} \\
\vdots \\
\int_{\mathbb{R}^{n}} L\left(\varphi^{\perp}\right) \hat{Z}_{\alpha h}
\end{array}\right]
$$

Due to the symmetries, the matrix $M$ has the form

$$
M=\left[\begin{array}{cc}
M_{1} & 0 \\
0 & M_{2}
\end{array}\right]
$$


where $M_{1}$ and $M_{2}$ are square matrices of dimensions $(5 \times(k+h+1))^{2}$ and $((n-4) \times(k+h+1))^{2}$ of the form

$$
M_{1}=\left[\begin{array}{ccccc}
\tilde{A} & \tilde{B} & \tilde{C} & \tilde{D} & \tilde{E} \\
\tilde{B}^{T} & \tilde{F} & \tilde{G} & \tilde{H} & \tilde{I} \\
\tilde{C}^{T} & \tilde{G}^{T} & \tilde{J} & \tilde{K} & \tilde{L} \\
\tilde{D}^{T} & \tilde{H}^{T} & \tilde{K}^{T} & \tilde{M} & \tilde{N} \\
\tilde{E}^{T} & \tilde{I}^{T} & \tilde{L}^{T} & \tilde{N}^{T} & \tilde{P}
\end{array}\right], \quad M_{2}=\left[\begin{array}{cccc}
\tilde{H}_{5} & 0 & 0 & 0 \\
0 & \tilde{H}_{6} & 0 & 0 \\
\ldots & \ldots & \ldots & \ldots \\
0 & 0 & 0 & \tilde{H}_{n}
\end{array}\right]
$$

with

$$
\tilde{H}_{\alpha}=\left[\begin{array}{ccc}
\int L\left(Z_{\alpha 0}\right) Z_{\alpha 0} & \left(\int L\left(Z_{\alpha 0}\right) \bar{Z}_{\alpha j}\right)_{j} & \left(\int L\left(Z_{\alpha 0}\right) \hat{Z}_{\alpha l}\right)_{l} \\
\left(\int L\left(\bar{Z}_{\alpha i}\right) Z_{\alpha 0}\right)_{i} & \left(\int L\left(\bar{Z}_{\alpha i}\right) \bar{Z}_{\alpha j}\right)_{i, j} & \left(\int L\left(\bar{Z}_{\alpha i}\right) \hat{Z}_{\alpha l}\right)_{i, l} \\
\left(\int L\left(\hat{Z}_{\alpha m}\right) Z_{\alpha 0}\right)_{m} & \left(\int L\left(\hat{Z}_{\alpha m}\right) \bar{Z}_{\alpha j}\right)_{m, j} & \left(\int L\left(\hat{Z}_{\alpha m}\right) \hat{Z}_{\alpha l}\right)_{m, l}
\end{array}\right]
$$

for $i, j=1, \ldots, k$ and $m, l=1, \ldots, h$. Thus, solving (2.0.34) is equivalent to find a solution of

$$
M_{1}\left[\begin{array}{l}
c_{0} \\
c_{1} \\
c_{2} \\
c_{3} \\
c_{4}
\end{array}\right]=\left[\begin{array}{l}
r_{0} \\
r_{1} \\
r_{2} \\
r_{3} \\
r_{4}
\end{array}\right], \quad \tilde{H}_{\alpha} c_{\alpha}=r_{\alpha} \text { for } \alpha=5, \ldots, n,
$$

with $r_{\alpha}$ defined in (2.0.34).

Proposition 2.0.3. There exists $k_{0}, h_{0}$ such that, for all $k>k_{0}, h>h_{0}$, system (2.0.36) is solvable. Moreover, the solution has the form

$$
\begin{aligned}
& c_{0}=v_{0}+t_{0}\left[\begin{array}{c}
1 \\
-\overline{1} \\
-\hat{1}
\end{array}\right]+t_{1}\left[\begin{array}{l}
0 \\
\overline{0} \\
\hat{0}
\end{array}\right]+t_{2}\left[\begin{array}{l}
0 \\
\overline{0} \\
\hat{0}
\end{array}\right]+t_{3}\left[\begin{array}{c}
0 \\
\overline{0} \\
\hat{0}
\end{array}\right]+t_{4}\left[\begin{array}{c}
0 \\
\overline{0} \\
\hat{0}
\end{array}\right] \\
& +\bar{t}_{0}\left[\begin{array}{l}
0 \\
\overline{0} \\
\hat{0}
\end{array}\right]+\bar{t}_{1}\left[\begin{array}{c}
0 \\
\overline{\cos } \\
\hat{0}
\end{array}\right]+\bar{t}_{2}\left[\begin{array}{c}
0 \\
\sin \\
\hat{0}
\end{array}\right]+\hat{t}_{0}\left[\begin{array}{c}
0 \\
\overline{0} \\
\hat{0}
\end{array}\right]+\hat{t}_{1}\left[\begin{array}{c}
0 \\
\overline{0} \\
\cos
\end{array}\right]+\hat{t}_{2}\left[\begin{array}{c}
0 \\
\overline{0} \\
\hat{\sin }
\end{array}\right], \\
& c_{1}=v_{1}+t_{0}\left[\begin{array}{c}
0 \\
-\overline{1} \\
\hat{0}
\end{array}\right]+t_{1}\left[\begin{array}{c}
1 \\
-\frac{1}{\sqrt{1-\mu^{2}}} \overline{\cos } \\
-\hat{1}
\end{array}\right]+t_{2}\left[\begin{array}{c}
0 \\
-\frac{1}{\sqrt{1-\mu^{2}}} \overline{\sin } \\
\hat{0}
\end{array}\right]+t_{3}\left[\begin{array}{c}
0 \\
\overline{0} \\
\hat{0}
\end{array}\right]+t_{4}\left[\begin{array}{c}
0 \\
\overline{0} \\
\hat{0}
\end{array}\right] \\
& +\bar{t}_{0}\left[\begin{array}{l}
0 \\
\overline{0} \\
\hat{0}
\end{array}\right]+\bar{t}_{1}\left[\begin{array}{c}
0 \\
-\overline{\cos } \\
\hat{0}
\end{array}\right]+\bar{t}_{2}\left[\begin{array}{c}
0 \\
-\overline{\sin } \\
\hat{0}
\end{array}\right]+\hat{t}_{0}\left[\begin{array}{c}
0 \\
\overline{0} \\
\hat{0}
\end{array}\right]+\hat{t}_{1}\left[\begin{array}{c}
0 \\
\overline{0} \\
\hat{0}
\end{array}\right]+\hat{t}_{2}\left[\begin{array}{c}
0 \\
\overline{0} \\
\hat{0}
\end{array}\right] \text {, } \\
& c_{2}=v_{2}+t_{0}\left[\begin{array}{l}
0 \\
\overline{0} \\
\hat{0}
\end{array}\right]+t_{1}\left[\begin{array}{c}
0 \\
\frac{1}{\sqrt{1-\mu^{2}}} \overline{\sin } \\
\hat{0}
\end{array}\right]+t_{2}\left[\begin{array}{c}
1 \\
-\frac{1}{\sqrt{1-\mu^{2}}} \overline{\cos } \\
-\hat{1}
\end{array}\right]+t_{3}\left[\begin{array}{l}
0 \\
\overline{0} \\
\hat{0}
\end{array}\right]+t_{4}\left[\begin{array}{l}
0 \\
\overline{0} \\
\hat{0}
\end{array}\right] \\
& +\bar{t}_{0}\left[\begin{array}{l}
0 \\
\overline{1} \\
\hat{0}
\end{array}\right]+\bar{t}_{1}\left[\begin{array}{l}
0 \\
\overline{0} \\
\hat{0}
\end{array}\right]+\bar{t}_{2}\left[\begin{array}{l}
0 \\
\overline{0} \\
\hat{0}
\end{array}\right]+\hat{t}_{0}\left[\begin{array}{c}
0 \\
\overline{0} \\
\hat{0}
\end{array}\right]+\hat{t}_{1}\left[\begin{array}{c}
0 \\
\overline{0} \\
\hat{0}
\end{array}\right]+\hat{t}_{2}\left[\begin{array}{c}
0 \\
\overline{0} \\
\hat{0}
\end{array}\right] \text {, }
\end{aligned}
$$




$$
\begin{aligned}
& c_{3}=v_{3}+t_{0}\left[\begin{array}{c}
0 \\
\overline{0} \\
-\hat{1}
\end{array}\right]+t_{1}\left[\begin{array}{l}
0 \\
\overline{0} \\
\hat{0}
\end{array}\right]+t_{2}\left[\begin{array}{l}
0 \\
\overline{0} \\
\hat{0}
\end{array}\right]+t_{3}\left[\begin{array}{c}
1 \\
-\overline{1} \\
-\frac{1}{\sqrt{1-\lambda^{2}}} \mathrm{côs}
\end{array}\right]+t_{4}\left[\begin{array}{c}
\frac{0}{0} \\
-\frac{1}{\sqrt{1-\lambda^{2}}} \mathrm{sin}
\end{array}\right] \\
& +\bar{t}_{0}\left[\begin{array}{l}
0 \\
\overline{0} \\
\hat{0}
\end{array}\right]+\bar{t}_{1}\left[\begin{array}{l}
0 \\
\overline{0} \\
\hat{0}
\end{array}\right]+\bar{t}_{2}\left[\begin{array}{l}
0 \\
\overline{0} \\
\hat{0}
\end{array}\right]+\hat{t}_{0}\left[\begin{array}{l}
0 \\
\overline{0} \\
\hat{0}
\end{array}\right]+\hat{t}_{1}\left[\begin{array}{c}
0 \\
\overline{0} \\
-\operatorname{coss}
\end{array}\right]+\hat{t}_{2}\left[\begin{array}{c}
0 \\
\overline{0} \\
-\hat{\sin }
\end{array}\right] \text {, } \\
& c_{4}=v_{4}+t_{0}\left[\begin{array}{l}
0 \\
\overline{0} \\
\hat{0}
\end{array}\right]+t_{1}\left[\begin{array}{l}
0 \\
\overline{0} \\
\hat{0}
\end{array}\right]+t_{2}\left[\begin{array}{l}
0 \\
\overline{0} \\
\hat{0}
\end{array}\right]+t_{3}\left[\begin{array}{c}
0 \\
\overline{0} \\
\frac{1}{\sqrt{1-\lambda^{2}}} \sin
\end{array}\right]+t_{4}\left[\begin{array}{c}
1 \\
-\overline{1} \\
-\frac{1}{\sqrt{1-\lambda^{2}}} \mathrm{côs}
\end{array}\right] \\
& +\bar{t}_{0}\left[\begin{array}{l}
0 \\
\overline{0} \\
\hat{0}
\end{array}\right]+\bar{t}_{1}\left[\begin{array}{l}
0 \\
\overline{0} \\
\hat{0}
\end{array}\right]+\bar{t}_{2}\left[\begin{array}{l}
0 \\
\overline{0} \\
\hat{0}
\end{array}\right]+\hat{t}_{0}\left[\begin{array}{l}
0 \\
\overline{0} \\
\hat{1}
\end{array}\right]+\hat{t}_{1}\left[\begin{array}{c}
0 \\
\overline{0} \\
\hat{0}
\end{array}\right]+\hat{t}_{2}\left[\begin{array}{c}
0 \\
\overline{0} \\
\hat{0}
\end{array}\right]
\end{aligned}
$$

and, for $\alpha=5, \ldots, n$,

$$
c_{\alpha}=v_{\alpha}+t_{\alpha}\left[\begin{array}{c}
1 \\
-\overline{1} \\
-\hat{1}
\end{array}\right]+\bar{\nu}_{\alpha 1}\left[\begin{array}{c}
0 \\
\overline{\cos } \\
\hat{0}
\end{array}\right]+\bar{\nu}_{\alpha 2}\left[\begin{array}{c}
\frac{0}{\sin } \\
\hat{0}
\end{array}\right]+\hat{\nu}_{\alpha 1}\left[\begin{array}{c}
0 \\
\overline{0} \\
\operatorname{côs}
\end{array}\right]+\hat{\nu}_{\alpha 2}\left[\begin{array}{c}
0 \\
\overline{0} \\
\hat{\sin }
\end{array}\right] \text {, }
$$

for any $t_{0}, t_{1}, t_{2}, t_{3}, t_{4}, \bar{t}_{0}, \bar{t}_{1}, \bar{t}_{2}, \hat{t}_{0}, \hat{t}_{1}, \hat{t}_{2}$, and $t_{\alpha}, \bar{\nu}_{\alpha 1}, \bar{\nu}_{\alpha 2}, \hat{\nu}_{\alpha 1}, \hat{\nu}_{\alpha 2}$ real parameters. The vectors $v_{\alpha} \in \mathbb{R}^{k+h+1}$ are fixed and satisfy

$$
\left\|v_{\alpha}\right\| \leqslant C\left\|\varphi^{\perp}\right\|, \alpha=0,1, \ldots, n .
$$

Proof. Proceeding as in [20, Proposition 6.1] it can be checked that, for any $\alpha=0, \ldots, n$,

$$
\left\|\bar{r}_{\alpha}\right\| \leqslant C \mu^{\frac{n-2}{2}}\left\|\varphi^{\perp}\right\|, \quad\left\|\hat{r}_{\alpha}\right\| \leqslant C \lambda^{\frac{n-2}{2}}\left\|\varphi^{\perp}\right\|,
$$

and combining this estimate with Lemma 2.1.1 and Lemma 2.2.2 we obtain the result.

We shall use the following notations: for any $\alpha=0,1, \ldots, n$,

$$
\begin{gathered}
\tilde{c}_{\alpha}:=\left[\begin{array}{c}
\bar{c}_{\alpha} \\
\hat{c}_{\alpha}
\end{array}\right] \in \mathbb{R}^{k+h}, \bar{c}_{\alpha}:=\left[\begin{array}{c}
\bar{c}_{\alpha 1} \\
\ldots \\
\bar{c}_{\alpha k}
\end{array}\right] \in \mathbb{R}^{k}, \hat{c}_{\alpha}:=\left[\begin{array}{c}
\hat{c}_{\alpha 1} \\
\ldots \\
\hat{c}_{\alpha h}
\end{array}\right] \in \mathbb{R}^{h}, \\
\tilde{r}_{\alpha}:=\left[\begin{array}{c}
\bar{r}_{\alpha} \\
\hat{r}_{\alpha}
\end{array}\right] \in \mathbb{R}^{k+h},
\end{gathered}
$$

where

$$
\bar{r}_{\alpha}:=\left[\begin{array}{c}
\int_{\mathbb{R}^{n}} L\left(\varphi^{\perp}\right) \bar{Z}_{\alpha 1} \\
\vdots \\
\int_{\mathbb{R}^{n}} L\left(\varphi^{\perp}\right) \bar{Z}_{\alpha k}
\end{array}\right] \in \mathbb{R}^{k}, \quad \hat{r}_{\alpha}:=\left[\begin{array}{c}
\int_{\mathbb{R}^{n}} L\left(\varphi^{\perp}\right) \hat{Z}_{\alpha 1} \\
\vdots \\
\int_{\mathbb{R}^{n}} L\left(\varphi^{\perp}\right) \hat{Z}_{\alpha h}
\end{array}\right] \in \mathbb{R}^{h} .
$$




\subsection{Solving THE SECOND SYSTEM IN (2.0.36)}

Let $\alpha$ be fixed in $\{5, \ldots, n\}$. This section is devoted to solve

$$
\tilde{H}_{\alpha} c_{\alpha}=r_{\alpha},
$$

where $r_{\alpha}$ is the vector defined in (2.0.34).

Using (2.0.35) and (2.0.6) and the fact that $L\left(\mathbf{z}_{\alpha}\right)=0$ it follows that

$$
\operatorname{row}_{1}\left(\tilde{H}_{\alpha}\right)=\sum_{l=2}^{k+h+1} \operatorname{row}_{l}\left(\tilde{H}_{\alpha}\right)
$$

As a consequence, $\left[\begin{array}{c}1 \\ -\overline{1} \\ -\hat{1}\end{array}\right] \in \operatorname{ker}\left(\tilde{H}_{\alpha}\right)$, and hence $\tilde{H}_{\alpha} c_{\alpha}=r_{\alpha}$ has a solution only if $r_{\alpha} \cdot\left[\begin{array}{c}1 \\ -\overline{1} \\ -\hat{1}\end{array}\right]=$ 0 . This last orthogonality condition is indeed fulfilled since one has

$$
\operatorname{row}_{1}\left(r_{\alpha}\right)=\sum_{j=2}^{k+1} \operatorname{row}_{j}\left(r_{\alpha}\right)+\sum_{l=k+2}^{h+k+1} \operatorname{row}_{l}\left(r_{\alpha}\right)
$$

again as consequence of the fact that $L\left(\mathbf{z}_{\alpha}\right)=0$. Thus, the general solution to (2.1.1) has the form

$$
c_{\alpha}=\left[\begin{array}{c}
0 \\
\tilde{c}_{\alpha}
\end{array}\right]+t\left[\begin{array}{c}
1 \\
-\overline{1} \\
-\hat{1}
\end{array}\right], \quad t \in \mathbb{R}
$$

where $\tilde{c}_{\alpha}$ solves

$$
H_{\alpha} \tilde{c}_{\alpha}=\tilde{r}_{\alpha},
$$

with

$$
H_{\alpha}=\left[\begin{array}{cc}
\bar{H}_{\alpha} & \gamma_{\alpha} \mathbb{1}_{k \times h} \\
\gamma_{\alpha} \mathbb{1}_{h \times k} & \hat{H}_{\alpha}
\end{array}\right], \quad \alpha=5, \ldots, n,
$$

being $\bar{H}_{\alpha}$ and $\hat{H}_{\alpha}$ square matrices of dimensions $k \times k$ and $h \times h$ respectively, defined by

$$
\bar{H}_{\alpha}:=\left(\int L\left(\bar{Z}_{\alpha, i}\right) \bar{Z}_{\alpha, j} d y\right)_{i, j=1, \ldots k}, \quad \hat{H}_{\alpha}:=\left(\int L\left(\hat{Z}_{\alpha, l}\right) \hat{Z}_{\alpha, m} d y\right)_{l, m=1, \ldots h}
$$

and

$$
\gamma_{\alpha}:=\int_{\mathbb{R}^{n}} L\left(\bar{Z}_{\alpha 1}\right) \hat{Z}_{\alpha 1}
$$

By $\mathbb{1}_{s \times t}$ we mean a $s \times t$-dimensional matrix whose entries are all 1 . Observe that

$$
\left|\gamma_{\alpha}\right| \leqslant C k^{4-2 n}
$$

for some fixed constant $C$. Arguing as in 20, one can show that $\bar{H}_{\alpha}$ and $\hat{H}_{\alpha}$ are circulant matrices of dimensions $(k \times k)$ and $(h \times h)$ respectively (see [17] for properties). Moreover, 20, Proposition 5.1] ensures that

$$
\bar{H}_{\alpha}\left[\bar{c}_{\alpha}\right]=\bar{s}_{\alpha}, \quad \hat{H}_{\alpha}\left[\hat{c}_{\alpha}\right]=\hat{s}_{\alpha},
$$

has a solution if

$$
\bar{s}_{\alpha} \cdot \overline{\cos }=\bar{s}_{\alpha} \cdot \overline{\sin }=0 \text { and } \hat{s}_{\alpha} \cdot \operatorname{côs}=\hat{s}_{\alpha} \cdot \hat{\sin }=0 \text {. }
$$

Actually, if a solution to (2.1.5) exists, it has the form

$$
\bar{c}_{\alpha}=\bar{w}_{\alpha}+\bar{\nu}_{1} \overline{\cos }+\bar{\nu}_{2} \overline{\sin }, \quad \hat{c}_{\alpha}=\hat{w}_{\alpha}+\hat{\nu}_{1} \cos +\hat{\nu}_{2} \sin ,
$$


for all $\bar{\nu}_{1}, \bar{\nu}_{2}, \hat{\nu}_{1}, \hat{\nu}_{2} \in \mathbb{R}$, where $\bar{w}_{\alpha}, \hat{w}_{\alpha}$ are the unique solutions to

$$
\bar{H}_{\alpha} \bar{w}_{\alpha}=\bar{s}_{\alpha}, \quad \bar{w}_{\alpha} \cdot \overline{\cos }=\bar{w}_{\alpha} \cdot \overline{\sin }=0
$$

and

$$
\hat{H}_{\alpha} \hat{w}_{\alpha}=\hat{s}_{\alpha}, \quad \hat{w}_{\alpha} \cdot \operatorname{côs}=\hat{w}_{\alpha} \cdot \hat{\sin }=0 .
$$

Furthermore, in [20, Proposition 5.1] it is proved that there exists a constant $C$ independent of $k$ so that, for all $k$ large

$$
\left\|\bar{w}_{\alpha}\right\| \leqslant C k^{n-4}\left\|\bar{s}_{\alpha}\right\| \text { and }\left\|\hat{w}_{\alpha}\right\| \leqslant C k^{n-4}\left\|\hat{s}_{\alpha}\right\| .
$$

We start with the observation that system (2.1.3) is solvable. Indeed, since $L\left(\mathbf{z}_{n+\alpha+4}\right)=$ $L\left(\mathbf{z}_{2 n+\alpha+2}\right)=0$, one has that $\bar{r}_{\alpha} \cdot \overline{\cos }=\bar{r}_{\alpha} \cdot \overline{\sin }=0$. Similarly, one gets that $\hat{r}_{\alpha} \cdot \cos =\hat{r}_{\alpha} \cdot \sin =0$, as consequence of the fact that $L\left(\mathbf{z}_{3 n+\alpha-2}\right)=L\left(\mathbf{z}_{4 n+\alpha-6}\right)=0$. Moreover, the vector $\mathbb{1}_{k \times h} \hat{c}_{\alpha}$ is a multiple of $\overline{1}$, and $\mathbb{1}_{h \times k} \bar{c}_{\alpha}$ is a multiple of $\hat{1}$. Thus $\mathbb{1}_{k \times h} \hat{c}_{\alpha} \cdot \cos =\mathbb{1}_{k \times h} \hat{c}_{\alpha} \cdot \sin =0$, and $\mathbb{1}_{h \times k} \bar{c}_{\alpha} \cdot \cos =\mathbb{1}_{h \times k} \bar{c}_{\alpha} \cdot \hat{\sin }=0$. Now we observe that the solution of system (2.1.3) has the form

$$
\begin{aligned}
& \bar{c}_{\alpha}=\bar{w}_{\alpha}+\bar{\nu}_{1} \overline{\cos }+\bar{\nu}_{2} \overline{\sin } \\
& \hat{c}_{\alpha}=\hat{w}_{\alpha}+\hat{\nu}_{1} \overline{\cos }+\hat{\nu}_{2} \overline{\sin },
\end{aligned}
$$

for any value for $\bar{\nu}_{1}, \bar{\nu}_{2}, \hat{\nu}_{1}, \hat{\nu}_{2} \in \mathbb{R}$, where $\bar{w}_{\alpha}$ and $\hat{w}_{\alpha}$ are the unique solutions to

$$
\begin{array}{ll}
\bar{H}_{\alpha} \bar{w}_{\alpha}=\bar{r}_{\alpha}-\gamma_{\alpha} \mathbb{1}_{k \times h} \hat{w}_{\alpha}, & \bar{w}_{\alpha} \cdot \overline{\cos }=\bar{w}_{\alpha} \cdot \overline{\sin }=0, \\
\hat{H}_{\alpha} \hat{w}_{\alpha}=\hat{r}_{\alpha}-\gamma_{\alpha} \mathbb{1}_{h \times k} \bar{w}_{\alpha}, & \hat{w}_{\alpha} \cdot \cos =\hat{w}_{\alpha} \cdot \sin =0 .
\end{array}
$$

Moreover, there exists a constant $C$ so that

$$
\left\|\bar{w}_{\alpha}\right\| \leqslant C k^{n-4}\left\|\bar{r}_{\alpha}\right\| \text { and }\left\|\hat{w}_{\alpha}\right\| \leqslant C k^{n-4}\left\|\hat{r}_{\alpha}\right\| .
$$

Existence and uniqueness of solutions to (2.1.7) satisfying (2.1.8) follows from a contraction map argument. Indeed, $\left[\begin{array}{l}\bar{w}_{\alpha} \\ \hat{w}_{\alpha}\end{array}\right]$ is a solution if and only if it is a fixed point to $A_{\alpha}\left[\begin{array}{c}\bar{w}_{\alpha} \\ \hat{w}_{\alpha}\end{array}\right]:=$ $T_{\alpha}^{-1}\left(\left[\begin{array}{c}\bar{r}_{\alpha}-\gamma_{\alpha} \mathbb{1}_{k \times h} \hat{w}_{\alpha} \\ \hat{r}_{\alpha}-\gamma_{\alpha} \mathbb{1}_{h \times k} \bar{w}_{\alpha}\end{array}\right]\right)$, where we denote by $T_{\alpha}$ the linear map $T_{\alpha}\left(\left[\begin{array}{c}\bar{w}_{\alpha} \\ \hat{w}_{\alpha}\end{array}\right]\right)=\left(\left[\begin{array}{c}\bar{H}_{\alpha} \bar{w}_{\alpha} \\ \hat{H}_{\alpha} \hat{w}_{\alpha}\end{array}\right]\right)$, which is invertible for vectors that are orthogonal to $\overline{\cos }, \overline{\sin }$, in their first components, and to

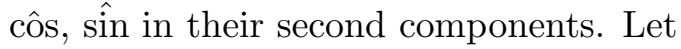

$$
B_{r}:=\left\{\left[\begin{array}{c}
\bar{w}_{\alpha} \\
\hat{w}_{\alpha}
\end{array}\right] \in K_{\alpha}:\left\|\bar{w}_{\alpha}\right\| \leqslant r k^{n-4}\left\|\bar{r}_{\alpha}\right\| \text { and }\left\|\hat{w}_{\alpha}\right\| \leqslant r k^{n-4}\left\|\hat{r}_{\alpha}\right\|\right\},
$$

where $K_{\alpha}:=\left\{\left[\begin{array}{c}\bar{w}_{\alpha} \\ \hat{w}_{\alpha}\end{array}\right] \in \mathbb{R}^{k+h}: \bar{w}_{\alpha} \cdot \overline{\cos }=\bar{w}_{\alpha} \cdot \overline{\sin }=0, \hat{w}_{\alpha} \cdot \cos =\hat{w}_{\alpha} \cdot \hat{\sin }=0\right\}$. Then, choosing $r$ large but fixed, and thanks to (2.1.4), one has that $A_{\alpha}$ is a contraction in $B_{r}$. This gives the existence of solutions to (2.1.7), satisfying (2.1.8).

Summarizing the above arguments, we have

Lemma 2.1.1. Let $\alpha \in\{5, \ldots, n\}$ be fixed. Then system (2.1.1) is solvable, and the solution has the form

$$
c_{\alpha}=\left[\begin{array}{c}
0 \\
\bar{w}_{\alpha} \\
\hat{w}_{\alpha}
\end{array}\right]+t\left[\begin{array}{c}
1 \\
-\overline{1} \\
-\hat{1}
\end{array}\right]+\bar{\nu}_{1}\left[\begin{array}{c}
0 \\
\overline{\cos } \\
\hat{0}
\end{array}\right]+\bar{\nu}_{2}\left[\begin{array}{c}
0 \\
\sin \\
\hat{0}
\end{array}\right]+\hat{\nu}_{1}\left[\begin{array}{c}
0 \\
\overline{0} \\
\operatorname{coss}
\end{array}\right]+\hat{\nu}_{2}\left[\begin{array}{c}
0 \\
\overline{0} \\
\hat{\sin }
\end{array}\right],
$$


for any values of $t, \bar{\nu}_{1}, \bar{\nu}_{2}, \hat{\nu}_{1}, \hat{\nu}_{2} \in \mathbb{R}$. In the above formula $\left[\begin{array}{c}\bar{w}_{\alpha} \\ \hat{w}_{\alpha}\end{array}\right]$ is the unique solution to (2.1.7), and satisfies (2.1.8).

\subsection{Solving The FIRST SYSTEM IN (2.0.36)}

This section is devoted to solve the first system in (2.0.36), namely

$$
M_{1}\left[\begin{array}{l}
c_{0} \\
c_{1} \\
c_{2} \\
c_{3} \\
c_{4}
\end{array}\right]=\left[\begin{array}{l}
r_{0} \\
r_{1} \\
r_{2} \\
r_{3} \\
r_{4}
\end{array}\right] .
$$

Using (2.0.35) and (2.0.6) and the fact that $L\left(\mathbf{z}_{\alpha}\right)=0$ for every $\alpha=0, \ldots, 4$, together with the result in Section 2.6, we observe that

$$
\begin{gathered}
\operatorname{row}_{1}\left(M_{1}\right)=\sum_{i=1}^{k} \operatorname{row}_{1+i}\left(M_{1}\right)+\operatorname{row}_{k+h+2+i}\left(M_{1}\right)+\sum_{i=1}^{h} \operatorname{row}_{k+1+i}\left(M_{1}\right)+\operatorname{row}_{4 k+3 h+4+i}\left(M_{1}\right), \\
\operatorname{row}_{k+h+2}\left(M_{1}\right)=\frac{1}{\sqrt{1-\mu^{2}}}\left[\sum_{i=1}^{k} \cos \bar{\theta}_{i} \operatorname{row}_{k+h+2+i}\left(M_{1}\right)-\sin \bar{\theta}_{i} \operatorname{row}_{2 k+2 h+3+i}\left(M_{1}\right)\right] \\
+\sum_{i=1}^{h} \operatorname{row}_{2 k+h+2+i}\left(M_{1}\right), \\
\operatorname{row}_{2 k+2 h+3}\left(M_{1}\right)=\frac{1}{\sqrt{1-\mu^{2}}}\left[\sum_{i=1}^{k} \sin \bar{\theta}_{i} \operatorname{row}_{k+h+2+i}\left(M_{1}\right)+\cos \bar{\theta}_{i} \operatorname{row}_{2 k+2 h+3+i}\left(M_{1}\right)\right] \\
+\sum_{i=1}^{h} \operatorname{row}_{3 k+2 h+3+i}\left(M_{1}\right), \\
\operatorname{row}_{3 k+3 h+4}\left(M_{1}\right)=\sum_{i=1}^{k} \operatorname{row}_{3 k+3 h+4+i}\left(M_{1}\right) \\
+\frac{1}{\sqrt{1-\lambda^{2}}}\left[\sum_{i=1}^{h} \cos _{i} \hat{\theta}_{i} \operatorname{row}_{4 k+3 h+4+i}\left(M_{1}\right)-\sin \hat{\theta}_{i} \operatorname{row}_{5 k+4 h+5+i}\left(M_{1}\right)\right] \\
\operatorname{row}_{4 k+4 h+5}\left(M_{1}\right)=\sum_{i=1}^{k} \operatorname{row}_{4 k+4 h+5+i}\left(M_{1}\right) \\
{\left[\sum_{i=1}^{h} \sin _{i} \hat{\operatorname{row}}_{4 k+3 h+4+i}\left(M_{1}\right)+\cos \hat{\theta}_{i} \operatorname{row}_{5 k+4 h+5+i}\left(M_{1}\right)\right]}
\end{gathered}
$$


From these facts we deduce that system (2.2.1) is solvable only if

$$
\left[\begin{array}{l}
r_{0} \\
r_{1} \\
r_{2} \\
r_{3} \\
r_{4}
\end{array}\right] \cdot w_{j}=0, \quad j=0,1, \ldots, 4
$$

where (recall definitions (2.0.12) and (2.0.13)

$$
w_{0}:=\left[\begin{array}{c}
1 \\
-\overline{1} \\
-\hat{1} \\
0 \\
-\overline{1} \\
\hat{0} \\
0 \\
\overline{0} \\
\hat{0} \\
0 \\
\overline{0} \\
-\hat{1} \\
0 \\
\overline{0} \\
\hat{0}
\end{array}\right], w_{1}:=\left[\begin{array}{c}
0 \\
\overline{0} \\
\hat{0} \\
1 \\
-\frac{1}{\sqrt{1-\mu^{2}}} \overline{\cos } \\
-\hat{1} \\
0 \\
\frac{1}{\sqrt{1-\mu^{2}}} \overline{\sin } \\
\hat{0} \\
0 \\
\overline{0} \\
\hat{0} \\
0 \\
\overline{0} \\
\hat{0}
\end{array}\right], w_{2}:=\left[\begin{array}{c}
0 \\
\overline{0} \\
\hat{0} \\
0 \\
-\frac{1}{\sqrt{1-\mu^{2}}} \overline{\sin } \\
\hat{0} \\
1 \\
\frac{1}{\sqrt{1-\mu^{2}}} \overline{\cos } \\
-\hat{1} \\
0 \\
\overline{0} \\
\hat{0} \\
0 \\
\overline{0} \\
\hat{0}
\end{array}\right],
$$

$$
w_{3}:=\left[\begin{array}{c}
0 \\
\overline{0} \\
\hat{0} \\
0 \\
\overline{0} \\
\hat{0} \\
0 \\
\overline{0} \\
\hat{0} \\
1 \\
-\overline{1} \\
-\frac{1}{\sqrt{1-\lambda^{2}}} \operatorname{côs} \\
0 \\
\overline{0} \\
\frac{1}{\sqrt{1-\lambda^{2}}} \sin
\end{array}\right], w_{4}:=\left[\begin{array}{c}
0 \\
\overline{0} \\
0 \\
\overline{0} \\
\hat{0} \\
0 \\
\overline{0} \\
\hat{0} \\
0 \\
\overline{0} \\
-\frac{1}{\sqrt{1-\lambda^{2}}} \sin \\
1 \\
-\overline{1} \\
-\frac{1}{\sqrt{1-\lambda^{2}}} \operatorname{côs}
\end{array}\right]
$$


which belong all to $\operatorname{ker}\left(M_{1}\right)$. On the other hand, using again that $L\left(\mathbf{z}_{\alpha}\right)=0$ for every $\alpha=$ $0, \ldots, 4$ one sees that the vectors $r_{\alpha}$ satisfy the following relations

$$
\begin{aligned}
\operatorname{row}_{1}\left(r_{0}\right) & =\sum_{j=2}^{k+1}\left[\operatorname{row}_{j}\left(r_{0}\right)+\operatorname{row}_{j}\left(r_{1}\right)\right]+\sum_{l=k+2}^{h+k+1}\left[\operatorname{row}_{l}\left(r_{0}\right)+\operatorname{row}_{l}\left(r_{3}\right)\right] \\
\operatorname{row}_{1}\left(r_{1}\right) & =\frac{1}{\sqrt{1-\mu^{2}}} \sum_{j=2}^{k+1}\left[\cos \bar{\theta}_{j-1} \operatorname{row}_{j}\left(r_{1}\right)-\sin \bar{\theta}_{j-1} \operatorname{row}_{j}\left(r_{2}\right)\right]+\sum_{l=k+2}^{h+k+1} \operatorname{row}_{l}\left(r_{1}\right), \\
\operatorname{row}_{1}\left(r_{2}\right) & =\frac{1}{\sqrt{1-\mu^{2}}} \sum_{j=2}^{k+1}\left[\sin \bar{\theta}_{j-1} \operatorname{row}_{j}\left(r_{1}\right)+\cos \bar{\theta}_{j-1} \operatorname{row}_{j}\left(r_{2}\right)\right]+\sum_{l=k+2}^{h+k+1} \operatorname{row}_{l}\left(r_{2}\right), \\
\operatorname{row}_{1}\left(r_{3}\right) & =\sum_{j=2}^{k+1} \operatorname{row}_{j}\left(r_{3}\right)+\frac{1}{\sqrt{1-\lambda^{2}}} \sum_{l=k+2}^{h+k+1}\left[\cos \hat{\theta}_{l-1} \operatorname{row}_{l}\left(r_{3}\right)-\sin \hat{\theta}_{l-1} \operatorname{row}_{l}\left(r_{4}\right)\right] \\
\operatorname{row}_{1}\left(r_{4}\right) & =\sum_{j=2}^{k+1} \operatorname{row}_{j}\left(r_{4}\right)+\frac{1}{\sqrt{1-\lambda^{2}}} \sum_{l=k+2}^{h+k+1}\left[\sin \hat{\theta}_{l-1} \operatorname{row}_{l}\left(r_{3}\right)+\cos \hat{\theta}_{l-1} \operatorname{row}_{l}\left(r_{4}\right)\right] .
\end{aligned}
$$

These facts imply that the orthogonality conditions (2.2.2) are satisfied, and thus (2.2.1) is solvable. The solution to (2.2.1) has the form

$$
\left[\begin{array}{c}
c_{0} \\
c_{1} \\
c_{2} \\
c_{3} \\
c_{4}
\end{array}\right]=\left[\begin{array}{c}
0 \\
\tilde{c}_{0} \\
0 \\
\tilde{c}_{1} \\
0 \\
\tilde{c}_{2} \\
0 \\
\tilde{c}_{3} \\
0 \\
\tilde{c}_{4}
\end{array}\right]+t w_{0}+s w_{1}+r w_{2}+u w_{3}+v w_{4}
$$

for any values of $t, s, r, u, v \in \mathbb{R}$, where $\tilde{c}_{\alpha}:=\left[\begin{array}{c}\bar{c}_{\alpha} \\ \hat{c}_{\alpha}\end{array}\right]$ are solutions of

$$
Q\left[\begin{array}{c}
\tilde{c}_{0} \\
\tilde{c}_{1} \\
\tilde{c}_{2} \\
\tilde{c}_{3} \\
\tilde{c}_{4}
\end{array}\right]=\left[\begin{array}{c}
\tilde{r}_{0} \\
\tilde{r}_{1} \\
\tilde{r}_{2} \\
\tilde{r}_{3} \\
\tilde{r}_{4}
\end{array}\right], \quad \tilde{r}_{\alpha}:=\left[\begin{array}{c}
\bar{r}_{\alpha} \\
\hat{r}_{\alpha}
\end{array}\right]
$$

Here $Q$ is the square matrix of dimension $[5(k+h) \times(k+h)]^{2}$ defined as

$$
Q:=\left[\begin{array}{ccccc}
A & B & C & D & E \\
B^{T} & F & G & H & I \\
C^{T} & G^{T} & J & K & L \\
D^{T} & H^{T} & K^{T} & M & N \\
E^{T} & I^{T} & L^{T} & N^{T} & P
\end{array}\right],
$$


where every submatrix of $Q$ has dimension $(k+h) \times(k+h)$ and entries of the form

$$
\int_{\mathbb{R}^{n}} L(V) W
$$

where

(i) In $A: V, W \in\left\{\left(\bar{Z}_{0 j}\right)_{j=1, \ldots, k},\left(\hat{Z}_{0 l}\right)_{l=1, \ldots, h}\right\}$.

(ii) In $B: V \in\left\{\left(\bar{Z}_{0 j}\right)_{j=1, \ldots, k},\left(\hat{Z}_{0 l}\right)_{l=1, \ldots, h}\right\}, W \in\left\{\left(\bar{Z}_{1 j}\right)_{j=1, \ldots, k},\left(\hat{Z}_{1 l}\right)_{l=1, \ldots, h}\right\}$.

(iii) In $C: V \in\left\{\left(\bar{Z}_{0 j}\right)_{j=1, \ldots, k},\left(\hat{Z}_{0 l}\right)_{l=1, \ldots, h}\right\}, W \in\left\{\left(\bar{Z}_{2 j}\right)_{j=1, \ldots, k},\left(\hat{Z}_{2 l}\right)_{l=1, \ldots, h}\right\}$.

(iv) In $D: V \in\left\{\left(\bar{Z}_{0 j}\right)_{j=1, \ldots, k},\left(\hat{Z}_{0 l}\right)_{l=1, \ldots, h}\right\}, W \in\left\{\left(\bar{Z}_{3 j}\right)_{j=1, \ldots, k},\left(\hat{Z}_{3 l}\right)_{l=1, \ldots, h}\right\}$.

(v) In $E: V \in\left\{\left(\bar{Z}_{0 j}\right)_{j=1, \ldots, k},\left(\hat{Z}_{0 l}\right)_{l=1, \ldots, h}\right\}, W \in\left\{\left(\bar{Z}_{4 j}\right)_{j=1, \ldots, k},\left(\hat{Z}_{4 l}\right)_{l=1, \ldots, h}\right\}$.

(vi) In $F: V, W \in\left\{\left(\bar{Z}_{1 j}\right)_{j=1, \ldots, k},\left(\hat{Z}_{1 l}\right)_{l=1, \ldots, h}\right\}$.

(vii) In $G: V \in\left\{\left(\bar{Z}_{1 j}\right)_{j=1, \ldots, k},\left(\hat{Z}_{1 l}\right)_{l=1, \ldots, h}\right\}, W \in\left\{\left(\bar{Z}_{2 j}\right)_{j=1, \ldots, k},\left(\hat{Z}_{2 l}\right)_{l=1, \ldots, h}\right\}$.

(viii) In $H: V \in\left\{\left(\bar{Z}_{1 j}\right)_{j=1, \ldots, k},\left(\hat{Z}_{1 l}\right)_{l=1, \ldots, h}\right\}, W \in\left\{\left(\bar{Z}_{3 j}\right)_{j=1, \ldots, k},\left(\hat{Z}_{3 l}\right)_{l=1, \ldots, h}\right\}$.

(ix) In $I: V \in\left\{\left(\bar{Z}_{1 j}\right)_{j=1, \ldots, k},\left(\hat{Z}_{1 l}\right)_{l=1, \ldots, h}\right\}, W \in\left\{\left(\bar{Z}_{4 j}\right)_{j=1, \ldots, k},\left(\hat{Z}_{4 l}\right)_{l=1, \ldots, h}\right\}$.

(x) In $J: V, W \in\left\{\left(\bar{Z}_{2 j}\right)_{j=1, \ldots, k},\left(\hat{Z}_{2 l}\right)_{l=1, \ldots, h}\right\}$.

(xi) In $K: V \in\left\{\left(\bar{Z}_{2 j}\right)_{j=1, \ldots, k},\left(\hat{Z}_{2 l}\right)_{l=1, \ldots, h}\right\}, W \in\left\{\left(\bar{Z}_{3 j}\right)_{j=1, \ldots, k},\left(\hat{Z}_{3 l}\right)_{l=1, \ldots, h}\right\}$.

(xii) In $L: V \in\left\{\left(\bar{Z}_{2 j}\right)_{j=1, \ldots, k},\left(\hat{Z}_{2 l}\right)_{l=1, \ldots, h}\right\}, W \in\left\{\left(\bar{Z}_{4 j}\right)_{j=1, \ldots, k},\left(\hat{Z}_{4 l}\right)_{l=1, \ldots, h}\right\}$.

(xiii) In $M: V, W \in\left\{\left(\bar{Z}_{3 j}\right)_{j=1, \ldots, k},\left(\hat{Z}_{3 l}\right)_{l=1, \ldots, h}\right\}$.

(xiv) In $N: V \in\left\{\left(\bar{Z}_{3 j}\right)_{j=1, \ldots, k},\left(\hat{Z}_{3 l}\right)_{l=1, \ldots, h}\right\}, W \in\left\{\left(\bar{Z}_{4 j}\right)_{j=1, \ldots, k},\left(\hat{Z}_{4 l}\right)_{l=1, \ldots, h}\right\}$.

(xv) In $P: V, W \in\left\{\left(\bar{Z}_{4 j}\right)_{j=1, \ldots, k},\left(\hat{Z}_{4 l}\right)_{l=1, \ldots, h}\right\}$.

Let us analize the structure of every matrix, where we will make use of the notation

$$
\begin{gathered}
\beta_{\alpha_{1}, \alpha_{2}}:=\int L\left(\bar{Z}_{\alpha_{1} 1}\right) \hat{Z}_{\alpha_{2} 1}, \\
\bar{\theta}_{j}:=\frac{2 \pi}{k}(j-1) \quad \text { and } \quad \hat{\theta}_{l}:=\frac{2 \pi}{h}(l-1) .
\end{gathered}
$$

Matrix A. Due to the invariance properties one can check that for $i, j=1, \ldots, k$ and $l, m=$ $1, \ldots, h$,

$$
\begin{aligned}
\int_{\mathbb{R}^{n}} L\left(\bar{Z}_{0 i}\right) \bar{Z}_{0 j} & =\int_{\mathbb{R}^{n}} L\left(\bar{Z}_{01}\right) \bar{Z}_{0,|i-j|+1}, \\
\int_{\mathbb{R}^{n}} L\left(\hat{Z}_{0 l}\right) \hat{Z}_{0 m} & =\int_{\mathbb{R}^{n}} L\left(\hat{Z}_{01}\right) \hat{Z}_{0,|l-m|+1}, \\
\int_{\mathbb{R}^{n}} L\left(\bar{Z}_{0 j}\right) \hat{Z}_{0 l} & =\int_{\mathbb{R}^{n}} L\left(\hat{Z}_{0 l}\right) \bar{Z}_{0 j}=\int_{\mathbb{R}^{n}} L\left(\bar{Z}_{01}\right) \hat{Z}_{01}=\beta_{00} .
\end{aligned}
$$

Thus we can write

$$
A=\left[\begin{array}{cc}
\bar{A} & A_{1}:=\beta_{00} \mathbb{1}_{k \times h} \\
A_{2}:=\beta_{00} \mathbb{1}_{h \times k} & \hat{A}
\end{array}\right],
$$

where $\bar{A}$ and $\hat{A}$ are circulant matrices of dimensions $(k \times k)$ and $(h \times h)$ (see [17]).

Matrix B. Applying the invariance properties like in matrix A we obtain

$$
B=\left[\begin{array}{cc}
\bar{B} & B_{1}:=\left(\int L\left(\bar{Z}_{0 j}\right) \hat{Z}_{1 l}\right)_{j=1, \ldots, k, l=1, \ldots, h} \\
B_{2}:=\left(\int L\left(\hat{Z}_{0 l}\right) \bar{Z}_{1 j}\right)_{l=1, \ldots, h, j=1, \ldots, k} & 0
\end{array}\right],
$$


where $\bar{B}$ is a $(k \times k)$ circulant matrix. Rotating in the $\left(y_{1}, y_{2}\right)$ and $\left(y_{3}, y_{4}\right)$ one obtains

$$
\begin{aligned}
& \int L\left(\bar{Z}_{0 j}\right) \hat{Z}_{1 l}=\cos \bar{\theta}_{j} \beta_{01}-\sin \bar{\theta}_{j} \beta_{02}=\cos \bar{\theta}_{j} \beta_{01}, \\
& \int L\left(\hat{Z}_{0 l}\right) \bar{Z}_{1 j}=\cos \bar{\theta}_{j} \beta_{10}-\sin \bar{\theta}_{j} \beta_{20}=\cos \bar{\theta}_{j} \beta_{10},
\end{aligned}
$$

for $j=1, \ldots, k, l=1, \ldots, h$, since $\beta_{02}=\beta_{20}=0$ due to the symmetry properties. Notice that both expressions are independent of $l$.

Matrix C. Likewise,

$$
C=\left[\begin{array}{cc}
\bar{C} & C_{1}:=\left(\int L\left(\bar{Z}_{0 j}\right) \hat{Z}_{2 l}\right)_{j=1, \ldots, k, l=1, \ldots, h} \\
C_{2}:=\left(\int L\left(\hat{Z}_{0 l}\right) \bar{Z}_{2 j}\right)_{l=1, \ldots, h, j=1, \ldots, k} & 0
\end{array}\right],
$$

whith $\bar{C}$ being a $(k \times k)$ circulant matrix and

$$
\begin{array}{ll}
\int L\left(\bar{Z}_{0 j}\right) \hat{Z}_{2 l}=\sin \bar{\theta}_{j} \beta_{01}, & j=1, \ldots, k, l=1, \ldots, h, \\
\int L\left(\hat{Z}_{0 l}\right) \bar{Z}_{2 j}=\sin \bar{\theta}_{j} \beta_{10}, & j=1, \ldots, k, l=1, \ldots, h .
\end{array}
$$

Matrix D.

$$
D=\left[\begin{array}{cc}
0 & D_{1}:=\left(\int L\left(\bar{Z}_{0 j}\right) \hat{Z}_{3 l}\right)_{j=1, \ldots, k, l=1, \ldots, h} \\
D_{2}:=\left(\int L\left(\hat{Z}_{0 l}\right) \bar{Z}_{3 j}\right)_{l=1, \ldots, h, j=1, \ldots, k} & \hat{D}
\end{array}\right],
$$

whith $\hat{D}$ being a $(h \times h)$ circulant matrix and

$$
\begin{aligned}
& \int L\left(\bar{Z}_{0 j}\right) \hat{Z}_{3 l}=\cos \hat{\theta}_{l} \beta_{03}, \quad j=1, \ldots, k, l=1, \ldots, h, \\
& \int L\left(\hat{Z}_{0 l}\right) \bar{Z}_{3 j}=\cos \hat{\theta}_{l} \beta_{30}, \quad j=1, \ldots, k, l=1, \ldots, h,
\end{aligned}
$$

where we have used that $\beta_{04}=\beta_{40}=0$.

Matrix E.

$$
E=\left[\begin{array}{cc}
0 & E_{1}:=\left(\int L\left(\bar{Z}_{0 j}\right) \hat{Z}_{4 l}\right)_{j=1, \ldots, k, l=1, \ldots, h} \\
E_{2}:=\left(\int L\left(\hat{Z}_{0 l}\right) \bar{Z}_{4 j}\right)_{l=1, \ldots, h, j=1, \ldots, k} & \hat{E}
\end{array}\right]
$$

whith $\hat{E}$ being a $(h \times h)$ circulant matrix and

$$
\begin{aligned}
& \int L\left(\bar{Z}_{0 j}\right) \hat{Z}_{4 l}=\sin \hat{\theta}_{l} \beta_{03}, \quad j=1, \ldots, k, l=1, \ldots, h, \\
& \int L\left(\hat{Z}_{0 l}\right) \bar{Z}_{4 j}=\sin \hat{\theta}_{l} \beta_{30}, \quad j=1, \ldots, k, l=1, \ldots, h .
\end{aligned}
$$


Matrix F.

$$
F=\left[\begin{array}{cc}
\bar{F} & F_{1}:=\left(\int L\left(\bar{Z}_{1 j}\right) \hat{Z}_{1 l}\right)_{j=1, \ldots, k, l=1, \ldots, h} \\
F_{2}:=\left(\int L\left(\hat{Z}_{1 l}\right) \bar{Z}_{1 j}\right)_{l=1, \ldots, h, j=1, \ldots, k} & \hat{F}
\end{array}\right],
$$

where $\bar{F}$ and $\hat{F}$ are $(k \times k)$ and $(h \times h)$ circulant matrices respectively and

$$
\int L\left(\bar{Z}_{1 j}\right) \hat{Z}_{1 l}=\int L\left(\hat{Z}_{1 l}\right) \bar{Z}_{1 j}=\cos ^{2} \bar{\theta}_{j} \beta_{11}+\sin ^{2} \bar{\theta}_{j} \beta_{22}
$$

for $j=1, \ldots, k, l=1, \ldots, h$, using that $\beta_{12}=\beta_{21}=0$.

\section{Matrix G.}

$$
G=\left[\begin{array}{cc}
\bar{G} & G_{1}:=\left(\int L\left(\bar{Z}_{1 j}\right) \hat{Z}_{2 l}\right)_{j=1, \ldots, k, l=1, \ldots, h} \\
G_{2}:=\left(\int L\left(\hat{Z}_{1 l}\right) \bar{Z}_{2 j}\right)_{l=1, \ldots, h, j=1, \ldots, k} & 0
\end{array}\right],
$$

where $\bar{G}$ is a $(k \times k)$ circulant matrix and

$$
\int L\left(\bar{Z}_{1 j}\right) \hat{Z}_{2 l}=\int L\left(\hat{Z}_{1 l}\right) \bar{Z}_{2 j}=\cos \bar{\theta}_{j} \sin \bar{\theta}_{j} \beta_{11}-\sin \bar{\theta}_{j} \cos \bar{\theta}_{j} \beta_{22},
$$

for $j=1, \ldots, k, l=1, \ldots, h$.

\section{Matrix $\mathbf{H}$.}

$$
H=\left[\begin{array}{cc}
0 & H_{1}:=\left(\int L\left(\bar{Z}_{1 j}\right) \hat{Z}_{3 l}\right)_{j=1, \ldots, k, l=1, \ldots, h} \\
H_{2}:=\left(\int L\left(\hat{Z}_{1 l}\right) \bar{Z}_{3 j}\right)_{l=1, \ldots, h, j=1, \ldots, k} & 0
\end{array}\right],
$$

where, since $\beta_{14}=\beta_{41}=\beta_{23}=\beta_{32}=\beta_{24}=\beta_{42}=0$,

$$
\begin{aligned}
& \int L\left(\bar{Z}_{1 j}\right) \hat{Z}_{3 l}=\cos \bar{\theta}_{j} \cos \hat{\theta}_{l} \beta_{13}, \\
& \int L\left(\hat{Z}_{1 l}\right) \bar{Z}_{3 j}=\cos \bar{\theta}_{j} \cos \hat{\theta}_{l} \beta_{31},
\end{aligned}
$$

for $j=1, \ldots, k, l=1, \ldots, h$.

\section{Matrix I.}

$$
I=\left[\begin{array}{cc}
0 & I_{1}:=\left(\int L\left(\bar{Z}_{1 j}\right) \hat{Z}_{4 l}\right)_{j=1, \ldots, k, l=1, \ldots, h} \\
I_{2}:=\left(\int L\left(\hat{Z}_{1 l}\right) \bar{Z}_{4 j}\right)_{l=1, \ldots, h, j=1, \ldots, k} & 0
\end{array}\right],
$$

where

$$
\begin{aligned}
& \int L\left(\bar{Z}_{1 j}\right) \hat{Z}_{4 l}=\cos \bar{\theta}_{j} \sin \hat{\theta}_{l} \beta_{13} \\
& \int L\left(\hat{Z}_{1 l}\right) \bar{Z}_{4 j}=\cos \bar{\theta}_{j} \sin \hat{\theta}_{l} \beta_{31}
\end{aligned}
$$

for $j=1, \ldots, k, l=1, \ldots, h$. 


\section{Matrix J.}

$$
J=\left[\begin{array}{cc}
\bar{J} & J_{1}:=\left(\int L\left(\bar{Z}_{2 j}\right) \hat{Z}_{2 l}\right)_{j=1, \ldots, k, l=1, \ldots, h} \\
J_{2}:=\left(\int L\left(\hat{Z}_{2 l}\right) \bar{Z}_{2 j}\right)_{l=1, \ldots, h, j=1, \ldots, k} & \hat{J}
\end{array}\right],
$$

where $\bar{J}$ and $\hat{J}$ are $(k \times k)$ and $(h \times h)$ circulant matrices respectively and

$$
\int L\left(\bar{Z}_{2 j}\right) \hat{Z}_{2 l}=\int L\left(\hat{Z}_{2 l}\right) \bar{Z}_{2 j}=\sin ^{2} \bar{\theta}_{j} \beta_{11}+\cos ^{2} \bar{\theta}_{j} \beta_{22}
$$

for $j=1, \ldots, k, l=1, \ldots, h$.

\section{Matrix K.}

$$
K=\left[\begin{array}{cc}
0 & K_{1}:=\left(\int L\left(\bar{Z}_{2 j}\right) \hat{Z}_{3 l}\right)_{j=1, \ldots, k, l=1, \ldots, h} \\
K_{2}:=\left(\int L\left(\hat{Z}_{2 l}\right) \bar{Z}_{3 j}\right)_{l=1, \ldots, h, j=1, \ldots, k} & 0
\end{array}\right],
$$

where

$$
\begin{aligned}
& \int L\left(\bar{Z}_{2 j}\right) \hat{Z}_{3 l}=\sin \bar{\theta}_{j} \cos \hat{\theta}_{l} \beta_{13}, \\
& \int L\left(\hat{Z}_{2 l}\right) \bar{Z}_{3 j}=\sin \bar{\theta}_{j} \cos \hat{\theta}_{l} \beta_{31},
\end{aligned}
$$

for $j=1, \ldots, k, l=1, \ldots, h$.

\section{Matrix L.}

$$
L=\left[\begin{array}{cc}
0 & L_{1}:=\left(\int L\left(\bar{Z}_{2 j}\right) \hat{Z}_{4 l}\right)_{j=1, \ldots, k, l=1, \ldots, h} \\
L_{2}:=\left(\int L\left(\hat{Z}_{2 l}\right) \bar{Z}_{4 j}\right)_{l=1, \ldots, h, j=1, \ldots, k} & 0
\end{array}\right],
$$

where

$$
\begin{aligned}
& \int L\left(\bar{Z}_{2 j}\right) \hat{Z}_{4 l}=\sin \bar{\theta}_{j} \sin \hat{\theta}_{l} \beta_{13}, \\
& \int L\left(\hat{Z}_{2 l}\right) \bar{Z}_{4 j}=\sin \bar{\theta}_{j} \sin \hat{\theta}_{l} \beta_{31},
\end{aligned}
$$

for $j=1, \ldots, k, l=1, \ldots, h$.

\section{Matrix M.}

$$
M=\left[\begin{array}{cc}
\bar{M} & M_{1}:=\left(\int L\left(\bar{Z}_{3 j}\right) \hat{Z}_{3 l}\right)_{j=1, \ldots, k, l=1, \ldots, h} \\
M_{2}:=\left(\int L\left(\hat{Z}_{3 l}\right) \bar{Z}_{3 j}\right)_{l=1, \ldots, h, j=1, \ldots, k} & \hat{M}
\end{array}\right],
$$

where $\bar{M}$ and $\hat{M}$ are $(k \times k)$ and $(h \times h)$ circulant matrices respectively and, since $\beta_{34}=\beta_{43}=0$,

$$
\int L\left(\bar{Z}_{3 j}\right) \hat{Z}_{3 l}=\int L\left(\hat{Z}_{3 l}\right) \bar{Z}_{3 j}=\cos ^{2} \hat{\theta}_{l} \beta_{33}+\sin ^{2} \hat{\theta}_{l} \beta_{44},
$$

for $j=1, \ldots, k, l=1, \ldots, h$. 


\section{Matrix N.}

$$
N=\left[\begin{array}{cc}
0 & N_{1}:=\left(\int L\left(\bar{Z}_{3 j}\right) \hat{Z}_{4 l}\right)_{j=1, \ldots, k, l=1, \ldots, h} \\
N_{2}:=\left(\int L\left(\hat{Z}_{3 l}\right) \bar{Z}_{4 j}\right)_{l=1, \ldots, h, j=1, \ldots, k} & \hat{N}
\end{array}\right],
$$

where $\hat{N}$ is a $(h \times h)$ circulant matrix and

$$
\int L\left(\bar{Z}_{3 j}\right) \hat{Z}_{4 l}=\int L\left(\hat{Z}_{3 l}\right) \bar{Z}_{4 j}=\cos \hat{\theta}_{l} \sin \hat{\theta}_{l} \beta_{33}-\sin \hat{\theta}_{l} \cos \hat{\theta}_{l} \beta_{44},
$$

for $j=1, \ldots, k, l=1, \ldots, h$.

\section{Matrix P.}

$$
P=\left[\begin{array}{cc}
\bar{P} & P_{1}:=\left(\int L\left(\bar{Z}_{4 j}\right) \hat{Z}_{4 l}\right)_{j=1, \ldots, k, l=1, \ldots, h} \\
P_{2}:=\left(\int L\left(\hat{Z}_{4 l}\right) \bar{Z}_{4 j}\right)_{l=1, \ldots, h, j=1, \ldots, k} & \hat{P}
\end{array}\right],
$$

where $\bar{P}$ and $\hat{P}$ are $(k \times k)$ and $(h \times h)$ circulant matrices respectively and

$$
\int L\left(\bar{Z}_{4 j}\right) \hat{Z}_{4 l}=\int L\left(\hat{Z}_{4 l}\right) \bar{Z}_{4 j}=\sin ^{2} \hat{\theta}_{l} \beta_{33}+\cos ^{2} \hat{\theta}_{l} \beta_{44}
$$

for $j=1, \ldots, k, l=1, \ldots, h$.

Notice that

$$
\hat{F}=\hat{J} \quad \text { and } \quad \bar{M}=\bar{P} .
$$

Henceforth, system (2.2.5) can be decomposed in two different systems in the following way,

$$
\begin{gathered}
{\left[\begin{array}{ccccc}
\bar{A} & \bar{B} & \bar{C} & 0 & 0 \\
\bar{B}^{T} & \bar{F} & \bar{G} & 0 & 0 \\
\bar{C}^{T} & \bar{G}^{T} & \bar{J} & 0 & 0 \\
0 & 0 & 0 & \bar{M} & 0 \\
0 & 0 & 0 & 0 & \bar{P}
\end{array}\right]\left[\begin{array}{c}
\bar{c}_{0} \\
\bar{c}_{1} \\
\bar{c}_{2} \\
\bar{c}_{3} \\
\bar{c}_{4}
\end{array}\right]=\left[\begin{array}{c}
\bar{r}_{0} \\
\bar{r}_{1} \\
\bar{r}_{2} \\
\bar{r}_{3} \\
\bar{r}_{4}
\end{array}\right]-\left[\begin{array}{ccccc}
A_{1} & B_{1} & C_{1} & D_{1} & E_{1} \\
B_{2}^{T} & F_{1} & G_{1} & H_{1} & I_{1} \\
C_{2}^{T} & G_{2}^{T} & J_{1} & K_{1} & L_{1} \\
D_{2}^{T} & H_{2}^{T} & K_{2}^{T} & M_{1} & N_{1} \\
E_{2}^{T} & I_{2}^{T} & L_{2}^{T} & N_{2}^{T} & P_{1}
\end{array}\right]\left[\begin{array}{l}
\hat{c}_{0} \\
\hat{c}_{1} \\
\hat{c}_{2} \\
\hat{c}_{3} \\
\hat{c}_{4}
\end{array}\right],} \\
{\left[\begin{array}{ccccc}
\hat{A} & 0 & 0 & \hat{D} & \hat{E} \\
0 & \hat{F} & 0 & 0 & 0 \\
0 & 0 & \hat{J} & 0 & 0 \\
\hat{D}^{T} & 0 & 0 & \hat{M} & \hat{N} \\
\hat{E}^{T} & 0 & 0 & \hat{N} & \hat{P}
\end{array}\right]\left[\begin{array}{l}
\hat{c}_{0} \\
\hat{c}_{1} \\
\hat{c}_{2} \\
\hat{c}_{3} \\
\hat{c}_{4}
\end{array}\right]=\left[\begin{array}{c}
\hat{r}_{0} \\
\hat{r}_{1} \\
\hat{r}_{2} \\
\hat{r}_{3} \\
\hat{r}_{4}
\end{array}\right]-\left[\begin{array}{ccccc}
A_{2} & B_{2} & C_{2} & D_{2} & E_{2} \\
B_{1}^{T} & F_{2} & G_{2} & H_{2} & I_{2} \\
C_{1}^{T} & G_{1}^{T} & J_{2} & K_{2} & L_{2} \\
D_{1}^{T} & H_{1}^{T} & K_{1}^{T} & M_{2} & N_{2} \\
E_{1}^{T} & I_{1}^{T} & L_{1}^{T} & N_{1}^{T} & P_{2}
\end{array}\right]\left[\begin{array}{c}
\bar{c}_{0} \\
\bar{c}_{1} \\
\bar{c}_{2} \\
\bar{c}_{3} \\
\bar{c}_{4}
\end{array}\right] .}
\end{gathered}
$$

By [20, Proposition 5.1] we know that the systems

$$
\left[\begin{array}{ccccc}
\bar{A} & \bar{B} & \bar{C} & 0 & 0 \\
\bar{B}^{T} & \bar{F} & \bar{G} & 0 & 0 \\
\bar{C}^{T} & \bar{G}^{T} & \bar{J} & 0 & 0 \\
0 & 0 & 0 & \bar{M} & 0 \\
0 & 0 & 0 & 0 & \bar{P}
\end{array}\right]\left[\begin{array}{c}
\bar{c}_{0} \\
\bar{c}_{1} \\
\bar{c}_{2} \\
\bar{c}_{3} \\
\bar{c}_{4}
\end{array}\right]=\left[\begin{array}{c}
\bar{s}_{0} \\
\bar{s}_{1} \\
\bar{s}_{2} \\
\bar{s}_{3} \\
\bar{s}_{4}
\end{array}\right]
$$




$$
\left[\begin{array}{ccccc}
\hat{A} & 0 & 0 & \hat{D} & \hat{E} \\
0 & \hat{F} & 0 & 0 & 0 \\
0 & 0 & \hat{J} & 0 & 0 \\
\hat{D}^{T} & 0 & 0 & \hat{M} & \hat{N} \\
\hat{E}^{T} & 0 & 0 & \hat{N}^{T} & \hat{P}
\end{array}\right]\left[\begin{array}{c}
\hat{c}_{0} \\
\hat{c}_{1} \\
\hat{c}_{2} \\
\hat{c}_{3} \\
\hat{c}_{4}
\end{array}\right]=\left[\begin{array}{c}
\hat{s}_{0} \\
\hat{s}_{1} \\
\hat{s}_{2} \\
\hat{s}_{3} \\
\hat{s}_{4}
\end{array}\right]
$$

are solvable if the orthogonality conditions

$$
\begin{aligned}
& \bar{s}_{2} \cdot \overline{1}=\left(\bar{s}_{0}+\bar{s}_{1}\right) \cdot \overline{\cos }=\left(\bar{s}_{0}+\bar{s}_{1}\right) \cdot \overline{\sin }=0, \\
& \bar{s}_{3} \cdot \overline{\cos }=\bar{s}_{3} \cdot \overline{\sin }=0, \\
& \bar{s}_{4} \cdot \overline{\cos }=\bar{s}_{4} \cdot \overline{\sin }=0, \\
& \hat{s}_{4} \cdot \hat{1}=\left(\hat{s}_{0}+\hat{s}_{3}\right) \cdot \operatorname{côs}=\left(\hat{s}_{0}+\hat{s}_{3}\right) \cdot \hat{\sin }=0, \\
& \hat{s}_{1} \cdot \operatorname{côs}=\hat{s}_{1} \cdot \hat{\sin }=0, \\
& \hat{s}_{2} \cdot \operatorname{côs}=\hat{s}_{2} \cdot \hat{\sin }=0,
\end{aligned}
$$

hold. Moreover,

$$
\begin{aligned}
& {\left[\begin{array}{l}
\bar{c}_{0} \\
\bar{c}_{1} \\
\bar{c}_{2}
\end{array}\right]=\left[\begin{array}{l}
\bar{w}_{0} \\
\bar{w}_{1} \\
\bar{w}_{2}
\end{array}\right]+\bar{t}_{1}\left[\begin{array}{l}
0 \\
0 \\
\overline{1}
\end{array}\right]+\bar{t}_{2}\left[\begin{array}{c}
\overline{\cos } \\
-\overline{\cos } \\
0
\end{array}\right]+\bar{t}_{3}\left[\begin{array}{c}
\overline{\sin } \\
-\overline{\sin } \\
0
\end{array}\right], \quad \forall \bar{t}_{1}, \bar{t}_{2}, \bar{t}_{3} \in \mathbb{R},} \\
& \bar{c}_{3}=\bar{w}_{3}+\bar{t}_{4} \overline{\cos }+\bar{t}_{5} \overline{\sin } \forall \bar{t}_{4}, \bar{t}_{5} \in \mathbb{R}, \\
& \bar{c}_{4}=\bar{w}_{4}+\bar{t}_{6} \overline{\cos }+\bar{t}_{7} \overline{\sin }, \forall \bar{t}_{6}, \bar{t}_{7} \in \mathbb{R}, \\
& \hat{c}_{1}=\hat{w}_{1}+\hat{t}_{1} \cos +\hat{t}_{2} \sin \forall \hat{t}_{1}, \hat{t}_{2} \in \mathbb{R}, \\
& \hat{c}_{2}=\hat{w}_{2}+\hat{t}_{3} \cos +\hat{t}_{4} \sin , \forall \hat{t}_{3}, \hat{t}_{4} \in \mathbb{R}, \\
& {\left[\begin{array}{l}
\hat{c}_{0} \\
\hat{c}_{3} \\
\hat{c}_{4}
\end{array}\right]=\left[\begin{array}{l}
\hat{w}_{0} \\
\hat{w}_{3} \\
\hat{w}_{4}
\end{array}\right]+\hat{t}_{5}\left[\begin{array}{l}
0 \\
0 \\
\hat{1}
\end{array}\right]+\hat{t}_{6}\left[\begin{array}{c}
\operatorname{coss} \\
-\operatorname{coss} \\
0
\end{array}\right]+\hat{t}_{7}\left[\begin{array}{c}
\sin \\
-\hat{\sin } \\
0
\end{array}\right], \forall \hat{t}_{5}, \hat{t}_{6}, \hat{t}_{7} \in \mathbb{R},}
\end{aligned}
$$

with $\left[\begin{array}{c}\bar{w}_{0} \\ \cdots \\ \bar{w}_{4}\end{array}\right],\left[\begin{array}{c}\hat{w}_{0} \\ \ldots \\ \hat{w}_{4}\end{array}\right]$ fixed vectors such that

$$
\left\|\left[\begin{array}{c}
\bar{w}_{0} \\
\ldots \\
\bar{w}_{4}
\end{array}\right]\right\| \leqslant \frac{C}{k^{n} \mu^{n-2}}\left\|\left[\begin{array}{c}
\bar{s}_{0} \\
\ldots \\
\bar{s}_{4}
\end{array}\right]\right\|, \quad\left\|\left[\begin{array}{c}
\hat{w}_{0} \\
\ldots \\
\hat{w}_{4}
\end{array}\right]\right\| \leqslant \frac{C}{h^{n} \lambda^{n-2}}\left\|\left[\begin{array}{c}
\hat{s}_{0} \\
\ldots \\
\hat{s}_{4}
\end{array}\right]\right\| .
$$


We will prove that (2.2.7) and (2.2.8) have a solution $\left[\begin{array}{c}\bar{c}_{0} \\ \hat{c}_{0} \\ \ldots \\ \bar{c}_{4} \\ \hat{c}_{4}\end{array}\right]$ in the space

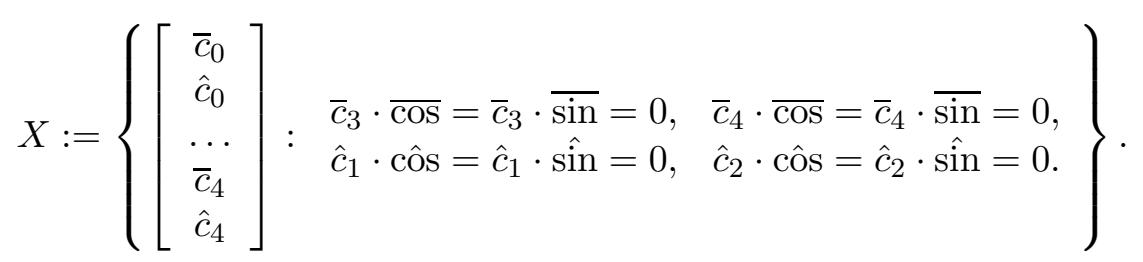

We need the following auxiliar result, whose proof follows straightforward using the same argument as in Lemma 1.2.2 so we skip it.

Lemma 2.2.1. Let $h, g$ be functions in $\mathbb{R}^{n}$ such that $h(y)=h\left(e^{\frac{2 \pi}{k}(j-1)} \bar{y}, \hat{y}, y^{\prime}\right)$ for all $j=1, \ldots, k$ and $g(y)=g\left(\bar{y}, e^{\frac{2 \pi}{h}(l-1)} \hat{y}, y^{\prime}\right)$ for all $l=1, \ldots, h$. Then,

$$
\begin{gathered}
\int_{\mathbb{R}^{n}} \hat{Z}_{1 l}(y) h(y) d y=\int_{\mathbb{R}^{n}} \hat{Z}_{2 l}(y) h(y) d y=0, \forall l=1, \ldots, h, \\
\int_{\mathbb{R}^{n}} \bar{Z}_{3 j}(y) g(y) d y=\int_{\mathbb{R}^{n}} \bar{Z}_{4 j}(y) g(y) d y=0, \forall j=1, \ldots, k .
\end{gathered}
$$

Let us focus on (2.2.7). By (2.0.9),

$$
\begin{aligned}
\bar{r}_{2} \cdot \overline{1} & =\sum_{j=1}^{k} \int L\left(\varphi^{\perp}\right) \bar{Z}_{2 j} \\
& =\sum_{j=1}^{k}\left[\int L\left(\sum_{\alpha=0}^{n} \sum_{i=1}^{k} \bar{Z}_{\alpha i}\right) \bar{Z}_{2 j}+\int L\left(\sum_{\alpha=0}^{n} \sum_{l=1}^{h} \hat{Z}_{\alpha l}\right) \bar{Z}_{2 j}\right] .
\end{aligned}
$$

Notice that the second term vanishes due to Lemma 2.2.1 since, by the symmetry properties of the functions,

$$
\sum_{j=1}^{k} \int L\left(\sum_{\alpha=0}^{n} \sum_{l=1}^{h} \hat{Z}_{\alpha l}\right) \bar{Z}_{2 j}=\sum_{j=1}^{k} \int L\left(\sum_{l=1}^{h} \hat{Z}_{2 l}\right) \bar{Z}_{2 j}=\sum_{l=1}^{h} \int \hat{Z}_{2 l} L\left(\sum_{j=1}^{k} \bar{Z}_{2 j}\right),
$$

and $L\left(\sum_{j=1}^{k} \bar{Z}_{2 j}\right)$ is invariant under rotation of angle $\frac{2 \pi}{k}(j-1)$ in the $\left(y_{1}, y_{2}\right)$ plane. Therefore,

$$
\bar{r}_{2} \cdot \overline{1}=\left(\int L\left(\sum_{\alpha=0}^{n} \sum_{i=1}^{k} \bar{Z}_{\alpha i}\right) \bar{Z}_{21}\right) \sum_{j=1}^{k} \sin \bar{\theta}_{j}+\left(\int L\left(\sum_{\alpha=0}^{k} \sum_{i=1}^{k} \bar{Z}_{\alpha i}\right) \bar{Z}_{22}\right) \sum_{j=1}^{k} \cos \bar{\theta}_{j}=0 .
$$

On the other hand, as a consequence of [20, Lemma 6.1],

$$
\left(\bar{r}_{0}+\bar{r}_{1}\right) \cdot \overline{\cos }=\left(\bar{r}_{0}+\bar{r}_{1}\right) \cdot \overline{\sin }=0 .
$$


Using the invariances under rotation in the planes $\left(y_{1}, y_{2}\right)$ and $\left(y_{3}, y_{4}\right)$ and Lemma 2.2.1 we get

$$
\begin{aligned}
\int L\left(\varphi^{\perp}\right) \bar{Z}_{3 j} & =\int L\left(\sum_{\alpha=1}^{n} \sum_{i=1}^{k} \bar{Z}_{\alpha i}\right) \bar{Z}_{3 j}+\int L\left(\sum_{\alpha=1}^{n} \sum_{l=1}^{h} \hat{Z}_{\alpha l}\right) \bar{Z}_{3 j} \\
& =\int L\left(\sum_{\alpha=1}^{n} \sum_{i=1}^{k} \bar{Z}_{\alpha i}\right) \bar{Z}_{31}
\end{aligned}
$$

and thus

$$
\bar{r}_{3} \cdot \overline{\cos }=\int L\left(\sum_{\alpha=1}^{n} \sum_{i=1}^{k} \bar{Z}_{\alpha i} \bar{Z}_{31}\left(\sum_{j=1}^{k} \cos \theta_{j}\right)=0 .\right.
$$

Analogously,

$$
\bar{r}_{3} \cdot \overline{\sin }=\bar{r}_{4} \cdot \overline{\cos }=\bar{r}_{4} \cdot \overline{\sin }=0 .
$$

Let us now check the last term in (2.2.7). We expect

$$
\begin{aligned}
& \left(C_{2}^{T}\left[\hat{c}_{0}\right]+G_{2}^{T}\left[\hat{c}_{1}\right]+J_{1}\left[\hat{c}_{2}\right]+K_{1}\left[\hat{c}_{3}\right]+L_{1}\left[\hat{c}_{4}\right]\right) \cdot \overline{1}=0, \\
& \left(A_{1}\left[\hat{c}_{0}\right]+B_{1}\left[\hat{c}_{1}\right]+C_{1}\left[\hat{c}_{2}\right]+D_{1}\left[\hat{c}_{3}\right]+E_{1}\left[\hat{c}_{4}\right]\right. \\
& \left.\quad+B_{2}^{T}\left[\hat{c}_{0}\right]+F_{1}\left[\hat{c}_{1}\right]+G_{1}\left[\hat{c}_{2}\right]+H_{1}\left[\hat{c}_{3}\right]+I_{1}\left[\hat{c}_{4}\right]\right) \cdot \overline{\cos }=0, \\
& \left(A_{1}\left[\hat{c}_{0}\right]+B_{1}\left[\hat{c}_{1}\right]+C_{1}\left[\hat{c}_{2}\right]+D_{1}\left[\hat{c}_{3}\right]+E_{1}\left[\hat{c}_{4}\right]\right. \\
& \left.\quad+B_{2}^{T}\left[\hat{c}_{0}\right]+F_{1}\left[\hat{c}_{1}\right]+G_{1}\left[\hat{c}_{2}\right]+H_{1}\left[\hat{c}_{3}\right]+I_{1}\left[\hat{c}_{4}\right]\right) \cdot \overline{\sin }=0, \\
& \left(D_{2}^{T}\left[\hat{c}_{0}\right]+H_{2}^{T}\left[\hat{c}_{1}\right]+K_{2}^{T}\left[\hat{c}_{2}\right]+M_{1}\left[\hat{c}_{3}\right]+N_{1}\left[\hat{c}_{4}\right]\right) \cdot \overline{\cos }=0, \\
& \left(D_{2}^{T}\left[\hat{c}_{0}\right]+H_{2}^{T}\left[\hat{c}_{1}\right]+K_{2}^{T}\left[\hat{c}_{2}\right]+M_{1}\left[\hat{c}_{3}\right]+N_{1}\left[\hat{c}_{4}\right]\right) \cdot \overline{\sin }=0, \\
& \left(E_{2}^{T}\left[\hat{c}_{0}\right]+I_{2}^{T}\left[\hat{c}_{1}\right]+L_{2}^{T}\left[\hat{c}_{2}\right]+N_{2}^{T}\left[\hat{c}_{3}\right]+P_{1}\left[\hat{c}_{4}\right]\right) \cdot \overline{\cos }=0, \\
& \left(E_{2}^{T}\left[\hat{c}_{0}\right]+I_{2}^{T}\left[\hat{c}_{1}\right]+L_{2}^{T}\left[\hat{c}_{2}\right]+N_{2}^{T}\left[\hat{c}_{3}\right]+P_{1}\left[\hat{c}_{4}\right]\right) \cdot \overline{\sin }=0,
\end{aligned}
$$

where $\hat{c}_{0}, \ldots, \hat{c}_{4}$ satisfy

$$
\begin{aligned}
& \hat{c}_{1} \cdot \operatorname{côs}=\hat{c}_{1} \cdot \hat{\sin }=0, \\
& \hat{c}_{2} \cdot \operatorname{côs}=\hat{c}_{2} \cdot \hat{\sin }=0 .
\end{aligned}
$$

Notice that, since $C_{2}$ and $G_{2}$ have all their rows identical,

$$
\begin{gathered}
C_{2}^{T}\left[\hat{c}_{0}\right] \cdot \overline{1}=\beta_{10}\left(\sum_{l=1}^{h} \hat{c}_{0 l}\right)\left(\sum_{j=1}^{k} \sin \bar{\theta}_{j}\right)=0, \\
G_{2}^{T}\left[\hat{c}_{1}\right] \cdot \overline{1}=\left(\beta_{11}-\beta_{22}\right)\left(\sum_{l=1}^{h} \hat{c}_{1 l}\right)\left(\sum_{j=1}^{k} \sin \bar{\theta}_{j} \cos \bar{\theta}_{j}\right)=0 .
\end{gathered}
$$

Likewise, using the definition of $J_{1}$ and Lemma 2.2.1,

$$
J_{1}\left[\hat{c}_{2}\right] \cdot \overline{1}=\left(\sum_{l=1}^{h} \hat{c}_{2 l}\right) \int L\left(\sum_{j=1}^{k} \bar{Z}_{2 j}\right) \hat{Z}_{11}=0
$$


since $L\left(\sum_{j=1}^{k} \bar{Z}_{2 j}\right)$ is invariant under rotation of angle $\theta_{j}$, and

$$
\begin{aligned}
& K_{1}\left[\hat{c}_{3}\right] \cdot \overline{1}=\beta_{13}\left(\hat{c}_{3} \cdot \operatorname{coss}\right)\left(\sum_{j=1}^{k} \sin \bar{\theta}_{j}\right)=0, \\
& L_{1}\left[\hat{c}_{4}\right] \cdot \overline{1}=\beta_{13}\left(\hat{c}_{4} \cdot \hat{\sin }\right)\left(\sum_{j=1}^{k} \sin \bar{\theta}_{j}\right)=0 .
\end{aligned}
$$

Thus, (2.2.16) follows. Furthermore, again by [20, Lemma 6.1],

$$
\begin{aligned}
\left(A_{1}+B_{2}^{T}\right)_{j l} & =\int L\left(\hat{Z}_{0 l}\right)\left(\bar{Z}_{0 j}+\bar{Z}_{1 j}\right)=0, \\
\left(B_{1}+F_{1}\right)_{j l} & =\int L\left(\hat{Z}_{1 l}\right)\left(\bar{Z}_{0 j}+\bar{Z}_{1 j}\right)=0, \\
\left(C_{1}+G_{1}\right)_{j l} & =\int L\left(\hat{Z}_{2 l}\right)\left(\bar{Z}_{0 j}+\bar{Z}_{1 j}\right)=0,
\end{aligned}
$$

and thus $\left(A_{1}+B_{2}^{T}\right)\left[\hat{c}_{0}\right]=\left(B_{1}+F_{1}\right)\left[\hat{c}_{1}\right]=\left(C_{1}+G_{1}\right)\left[\hat{c}_{2}\right]=0$. Likewise,

$$
\begin{aligned}
\left(D_{1}+H_{1}\right)_{j l} & =\int L\left(\hat{Z}_{3 l}\right)\left(\bar{Z}_{0 j}+\bar{Z}_{1 j}\right)=0, \\
\left(E_{1}+I_{1}\right)_{j l} & =\int L\left(\hat{Z}_{4 l}\right)\left(\bar{Z}_{0 j}+\bar{Z}_{1 j}\right)=0,
\end{aligned}
$$

and therefore $\left(D_{1}+H_{1}\right)\left[\hat{c}_{3}\right]=\left(E_{1}+I_{1}\right)\left[\hat{c}_{4}\right]=0$. (2.2.18) can be analogously proved. Furthermore,

$$
\begin{gathered}
D_{2}^{T}\left[\hat{c}_{0}\right] \cdot \overline{\cos }=\beta_{31}\left(\hat{c}_{0} \cdot \cos \right)\left(\sum_{j=1}^{k} \cos \bar{\theta}_{j}\right)=0, \\
M_{1}\left[\hat{c}_{3}\right] \cdot \overline{\cos }=\left[\beta_{33}\left(\sum_{l=1}^{h} \hat{c}_{3 l} \cos ^{2} \hat{\theta}_{l}\right)+\beta_{44}\left(\sum_{l=1}^{h} \hat{c}_{3 l} \sin ^{2} \hat{\theta}_{l}\right)\right]\left(\sum_{j=1}^{k} \cos \bar{\theta}_{j}\right)=0, \\
N_{1}\left[\hat{c}_{4}\right] \cdot \overline{\cos }=\left(\beta_{33}-\beta_{44}\right)\left(\sum_{l=1}^{h} \hat{c}_{4 l} \sin \hat{\theta}_{l} \cos \hat{\theta}_{l}\right)\left(\sum_{j=1}^{k} \cos \bar{\theta}_{j}\right)=0,
\end{gathered}
$$

and, due to (2.2.23),

$$
\begin{gathered}
H_{2}^{T}\left[\hat{c}_{1}\right] \cdot \overline{\cos }=\beta_{31}\left(\hat{c}_{1} \cdot \operatorname{coss}\right)\left(\sum_{j=1}^{k} \cos ^{2} \bar{\theta}_{j}\right)=0, \\
K_{2}^{T}\left[\hat{c}_{2}\right] \cdot \overline{\cos }=\beta_{31}\left(\hat{c}_{2} \cdot \operatorname{côs}\right)\left(\sum_{j=1}^{k} \sin \bar{\theta}_{j} \cos \bar{\theta}_{j}\right)=0,
\end{gathered}
$$

so (2.2.19) holds. Identities (2.2.20)-(2.2.22) can be obtained in a similar way, and thus (2.2.7) is solvable. An analogous reasoning proves the solvability of (2.2.8). Thus, the systems (2.2.7) 
and (2.2.8) have a solution in $X$ with the form (2.2.10), where $\left[\bar{w}_{0}, \ldots, \bar{w}_{4}, \hat{w}_{0}, \ldots, \hat{w}_{4}\right]$ satisfies (2.2.9). It can be checked that

$$
\begin{gathered}
\left|\beta_{\alpha \alpha}\right| \leqslant C k^{-2 n+4}, \alpha=0,2,4, \quad\left|\beta_{\alpha \alpha}\right| \leqslant C k^{-2 n+6}, \alpha=1,3, \\
\left|\beta_{\alpha_{1} \alpha_{2}}\right| \leqslant C k^{-2 n+6}, \alpha_{1}, \alpha_{2}=0,1,3, \alpha_{1} \neq \alpha_{2} .
\end{gathered}
$$

where $\beta_{\alpha_{1}, \alpha_{2}}$ was defined in (2.2.6), and henceforth, by (2.2.11) and recalling that $h=O(k)$,

$$
\left\|\bar{w}_{\alpha}\right\| \leqslant C k^{n-4}\left\|\bar{r}_{\alpha}\right\|, \quad\left\|\hat{w}_{\alpha}\right\| \leqslant C k^{n-4}\left\|\hat{r}_{\alpha}\right\|, \quad \alpha=0,1, \ldots, 4 .
$$

As it was done in the case $\alpha \geqslant 5$, we will solve the systems by means of a fixed point argument. If we denote

$$
\begin{gathered}
\bar{M}_{1}:=\left[\begin{array}{ccccc}
\bar{A} & \bar{B} & \bar{C} & 0 & 0 \\
\bar{B}^{T} & \bar{F} & \bar{G} & 0 & 0 \\
\bar{C}^{T} & \bar{G}^{T} & \bar{J} & 0 & 0 \\
0 & 0 & 0 & \bar{M} & 0 \\
0 & 0 & 0 & 0 & \bar{P}
\end{array}\right], \bar{M}_{1}:=\left[\begin{array}{ccccc}
A_{1} & B_{1} & C_{1} & D_{1} & E_{1} \\
B_{2}^{T} & F_{1} & G_{1} & H_{1} & I_{1} \\
C_{2}^{T} & G_{2}^{T} & J_{1} & K_{1} & L_{1} \\
D_{2}^{T} & H_{2}^{T} & K_{2}^{T} & M_{1} & N_{1} \\
E_{2}^{T} & I_{2}^{T} & L_{2}^{T} & N_{2}^{T} & P_{1}
\end{array}\right], \\
\hat{M}_{1}:=\left[\begin{array}{ccccc}
\hat{A} & 0 & 0 & \hat{D} & \hat{E} \\
0 & \hat{F} & 0 & 0 & 0 \\
0 & 0 & \hat{J} & 0 & 0 \\
\hat{D}^{T} & 0 & 0 & \hat{M} & \hat{N} \\
\hat{E}^{T} & 0 & 0 & \hat{N}^{T} & \hat{P}
\end{array}\right], \hat{\hat{M}}_{1}:=\left[\begin{array}{ccccc}
A_{2} & B_{2} & C_{2} & D_{2} & E_{2} \\
B_{1}^{T} & F_{2} & G_{2} & H_{2} & I_{2} \\
C_{1}^{T} & G_{1}^{T} & J_{2} & K_{2} & L_{2} \\
D_{1}^{T} & H_{1}^{T} & K_{1}^{T} & M_{2} & N_{2} \\
E_{1}^{T} & I_{1}^{T} & L_{1}^{T} & N_{1}^{T} & P_{2}
\end{array}\right],
\end{gathered}
$$

then $\left[\bar{w}_{0}, \ldots, \bar{w}_{4}, \hat{w}_{0}, \ldots, \hat{w}_{4}\right]$ is a solution of (2.2.7)-(2.2.8) if and only if it is a fixed point of

$$
F\left[\begin{array}{c}
\bar{w}_{0} \\
\ldots \\
\bar{w}_{4} \\
\hat{w}_{0} \\
\cdots \\
\hat{w}_{4}
\end{array}\right]:=S^{-1}\left(\left[\begin{array}{c}
\bar{r}_{0} \\
\bar{r}_{1} \\
\bar{r}_{2} \\
\bar{r}_{3} \\
\bar{r}_{4}
\end{array}\right]-\overline{\bar{M}}_{1}\left[\begin{array}{c}
\hat{w}_{0} \\
\hat{w}_{1} \\
\hat{w}_{2} \\
\hat{w}_{3} \\
\hat{w}_{4}
\end{array}\right],\left[\begin{array}{c}
\hat{r}_{0} \\
\hat{r}_{1} \\
\hat{r}_{2} \\
\hat{r}_{3} \\
\hat{r}_{4}
\end{array}\right]-\hat{\hat{M}}_{1}\left[\begin{array}{c}
\bar{w}_{0} \\
\bar{w}_{1} \\
\bar{w}_{2} \\
\bar{w}_{3} \\
\bar{w}_{4}
\end{array}\right]\right),
$$

where

$$
S\left(\left[\begin{array}{c}
\bar{w}_{0} \\
\bar{w}_{1} \\
\bar{w}_{2} \\
\bar{w}_{3} \\
\bar{w}_{4}
\end{array}\right],\left[\begin{array}{c}
\hat{w}_{0} \\
\hat{w}_{1} \\
\hat{w}_{2} \\
\hat{w}_{3} \\
\hat{w}_{4}
\end{array}\right]\right):=\left(\bar{M}_{1}\left[\begin{array}{c}
\bar{w}_{0} \\
\bar{w}_{1} \\
\bar{w}_{2} \\
\bar{w}_{3} \\
\bar{w}_{4}
\end{array}\right], \hat{M}_{1}\left[\begin{array}{c}
\hat{w}_{0} \\
\hat{w}_{1} \\
\hat{w}_{2} \\
\hat{w}_{3} \\
\hat{w}_{4}
\end{array}\right]\right) .
$$

Notice that $S$ is a linear map which is invertible for vectors satisfying the orthogonality conditions (2.2.9). Let

$$
\begin{gathered}
B_{r}:=\left\{\left[\begin{array}{c}
\bar{w}_{0} \\
\ldots \\
\bar{w}_{4}
\end{array}\right],\left[\begin{array}{c}
\hat{w}_{0} \\
\ldots \\
\hat{w}_{4}
\end{array}\right] \in K:\left\|\left[\begin{array}{c}
\bar{w}_{0} \\
\ldots \\
\bar{w}_{4}
\end{array}\right]\right\| \leqslant r k^{n-4}\left\|\left[\begin{array}{c}
\bar{r}_{0} \\
\ldots \\
\bar{r}_{4}
\end{array}\right]\right\|,\right. \\
\left.\left\|\left[\begin{array}{c}
\hat{w}_{0} \\
\ldots \\
\hat{w}_{4}
\end{array}\right]\right\| \leqslant r k^{n-4} \|\left[\begin{array}{c}
\hat{r}_{0} \\
\ldots \\
\hat{r}_{4}
\end{array}\right]\right\},
\end{gathered}
$$


for some fixed $r$ large, where

$$
\left.K:=\left\{\left[\begin{array}{c}
\bar{w}_{0} \\
\cdots \\
\bar{w}_{4}
\end{array}\right] \in \mathbb{R}^{5 \times k},\left[\begin{array}{c}
\hat{w}_{0} \\
\cdots \\
\hat{w}_{4}
\end{array}\right] \in \mathbb{R}^{5 \times h} \text { satisfying (2.2.9) }\right)\right\} .
$$

Thanks to the particular form of the matrices $\overline{\bar{M}}_{1}, \hat{\hat{M}}$ (all their submatrices are combinations of sinus and cosinus multiplied by a term $\beta_{\alpha_{1}, \alpha_{2}}$ ) and (2.2.24) it can be checked that $F$ is a contraction mapping that sends $B_{r}$ into $B_{r}$. This finishes the proof of the existence of a solution to (2.2.7)-(2.2.8) satisfying (2.2.9).

Define the vectors

$$
\begin{aligned}
& \bar{u}_{0}:=\left[\begin{array}{c}
0 \\
\overline{0} \\
\hat{0} \\
0 \\
\overline{0} \\
\hat{0} \\
0 \\
\overline{1} \\
\hat{0} \\
0 \\
\overline{0} \\
\hat{0} \\
0 \\
\overline{0} \\
\hat{0}
\end{array}\right], \bar{u}_{1}:=\left[\begin{array}{c}
0 \\
\overline{\cos } \\
\hat{0} \\
0 \\
-\overline{\cos } \\
\hat{0} \\
0 \\
\overline{0} \\
\hat{0} \\
0 \\
\overline{0} \\
\hat{0} \\
0 \\
\overline{0} \\
\hat{0}
\end{array}\right], \bar{u}_{2}:=\left[\begin{array}{c}
\frac{0}{\sin } \\
\hat{0} \\
\frac{0}{-\overline{\sin }} \\
\hat{0} \\
0 \\
\overline{0} \\
\hat{0} \\
0 \\
\overline{0} \\
\hat{0} \\
0 \\
\overline{0} \\
\hat{0}
\end{array}\right], \\
& \hat{u}_{0}:=\left[\begin{array}{c}
0 \\
\overline{0} \\
\hat{0} \\
0 \\
\overline{0} \\
\hat{0} \\
0 \\
\overline{0} \\
\hat{0} \\
0 \\
\overline{0} \\
\hat{0} \\
0 \\
\overline{0} \\
\hat{1}
\end{array}\right], \hat{u}_{1}:=\left[\begin{array}{c}
0 \\
\overline{0} \\
\text { côs } \\
0 \\
\overline{0} \\
\hat{0} \\
0 \\
\overline{0} \\
\hat{0} \\
0 \\
\overline{0} \\
-\operatorname{côs} \\
0 \\
\overline{0} \\
\hat{0}
\end{array}\right], \hat{u}_{2}:=\left[\begin{array}{c}
0 \\
\overline{0} \\
\hat{\sin } \\
0 \\
\overline{0} \\
\hat{0} \\
0 \\
\overline{0} \\
\hat{0} \\
0 \\
\overline{0} \\
-\sin \\
0 \\
\overline{0} \\
\hat{0}
\end{array}\right] \text {. }
\end{aligned}
$$

We can summarize this section in the following lemma. 
Lemma 2.2.2. System (2.2.1) is solvable, and the solution has the form

$$
\begin{aligned}
{\left[\begin{array}{c}
\tilde{c}_{0} \\
\ldots \\
\tilde{c}_{4}
\end{array}\right]=} & w+t_{0} w_{0}+t_{1} w_{1}+t_{2} w_{2}+t_{3} w_{3}+t_{4} w_{4} \\
& +\bar{t}_{0} \bar{u}_{0}+\bar{t}_{1} \bar{u}_{1}+\bar{t}_{2} \bar{u}_{2}+\hat{t}_{0} \hat{u}_{0}+\hat{t}_{1} \hat{u}_{1}+\hat{t}_{2} \hat{u}_{2},
\end{aligned}
$$

where

$$
w:=\left[\begin{array}{lllllllllllllll}
0 & \bar{w}_{0} & \hat{w}_{0} & 0 & \bar{w}_{1} & \hat{w}_{1} & 0 & \bar{w}_{2} & \hat{w}_{2} & 0 & \bar{w}_{3} & \hat{w}_{3} & 0 & \bar{w}_{4} & \hat{w}_{4}
\end{array}\right]^{T}
$$

$\left(\bar{w}_{0}, \ldots, \bar{w}_{4}, \hat{w}_{0}, \ldots, \hat{w}_{4}\right.$ being the unique solution to the system (2.2.7)-(2.2.8) satisfying (2.2.9) and (2.2.11) $), t_{0}, \ldots, t_{4}, \bar{t}_{0}, \bar{t}_{1}, \bar{t}_{2}, \hat{t}_{0}, \hat{t}_{1}, \hat{t}_{2} \in \mathbb{R}$ and $\tilde{c}_{0}, \ldots, \tilde{c}_{4}, w_{0}, \ldots, w_{4}, \bar{u}_{0}, \bar{u}_{1}, \bar{u}_{2}, \hat{u}_{0}, \hat{u}_{1}, \hat{u}_{2}$ are defined in (2.0.37), (2.2.3), (2.2.4), (2.2.25) and (2.2.26).

\subsection{Proof of Proposition 2.0.2}

With this in mind, we observe that Proposition 2.0.2 is a consequence of the following estimates: if $k, h \rightarrow \infty$

$$
\begin{aligned}
\int|u|^{p-1} Z_{\alpha 0} Z_{\beta 0} & =\int U^{p-1} Z_{00}^{2}+O\left(\mu^{\frac{n-2}{2}}+\lambda^{\frac{n-2}{2}}\right) \quad \text { if } \alpha=\beta=0, \\
& =\int U^{p-1} Z_{10}^{2}+O\left(\mu^{\frac{n-2}{2}}+\lambda^{\frac{n-2}{2}}\right) \quad \text { if } \alpha=\beta \neq 0, \\
& =O\left(\mu^{\frac{n-2}{2}}+\lambda^{\frac{n-2}{2}}\right) \quad \text { otherwise, } \\
\int|u|^{p-1} \bar{Z}_{\alpha i} \bar{Z}_{\beta j} & =\int U^{p-1} Z_{00}^{2}+O\left(\mu^{\frac{n-2}{2}}\right) \quad \text { if } \alpha=\beta=0, i=j, \\
& =\int U^{p-1} Z_{10}^{2}+O\left(\mu^{\frac{n-2}{2}}\right) \quad \text { if } \alpha=\beta \neq 0, i=j \\
& =O\left(\mu^{\frac{n-2}{2}}\right) \quad \text { otherwise, } \\
\int|u|^{p-1} \hat{Z}_{\alpha l} \hat{Z}_{\beta m} & =\int U^{p-1} Z_{00}^{2}+O\left(\lambda^{\frac{n-2}{2}}\right) \quad \text { if } \alpha=\beta=0, l=m, \\
& =\int U^{p-1} Z_{10}^{2}+O\left(\lambda^{\frac{n-2}{2}}\right) \quad \text { if } \alpha=\beta \neq 0, l=m, \\
& =O\left(\lambda^{\frac{n-2}{2}}\right) \quad \text { otherwise, } \\
\int|u|^{p-1} \bar{Z}_{\alpha i} Z_{\beta 0}=O\left(\mu^{\frac{n-2}{2}}\right), \quad \int|u|^{p-1} \hat{Z}_{\alpha l} Z_{\beta 0}=O\left(\lambda^{\frac{n-2}{2}}\right), & \\
& \int|u|^{p-1} \bar{Z}_{\alpha i} \hat{Z}_{\beta l}=O\left(\mu^{\frac{n-2}{2}} \lambda^{\frac{n-2}{2}}\right) .
\end{aligned}
$$

In the formulas above, $i, j=1, \ldots, k, l, m=1, \ldots, h, \alpha, \beta=0, \ldots, n$.

The proof of (2.3.2) and (2.3.3) follows like (8.3) in [20, and (2.3.1), (2.3.4) are obtained analogously. Let us prove (2.3.5). 
The key point here is to notice that if $y \in B\left(\xi_{i}, \frac{\bar{\alpha}}{k}\right)$ then $\left|y-\eta_{l}\right| \geqslant C$, with $C$ independent of $i$, $l$ and $k$, and $\left|y-\xi_{i}\right| \geqslant C$ whenever $y \in B\left(\eta_{l}, \frac{\hat{\alpha}}{h}\right)$, where $C$ is independent of $i, l$ and $h$. Consider the case $\alpha=\beta=0$. We split the integral into four parts.

$$
\begin{aligned}
\int|u|^{p-1} \bar{Z}_{0 i} \hat{Z}_{0 l}= & \int_{B\left(\xi_{i}, \frac{\bar{\alpha}}{k}\right)}|u|^{p-1} \bar{Z}_{0 i} \hat{Z}_{0 j}+\int_{B\left(\eta_{l}, \frac{\hat{\alpha}}{h}\right)}|u|^{p-1} \bar{Z}_{0 i} \hat{Z}_{0 j} \\
& +\int_{\mathbb{R}^{n} \backslash B(0,2)}|u|^{p-1} \bar{Z}_{0 i} \hat{Z}_{0 j} \\
& +\int_{B(0,2) \backslash\left(B\left(\xi_{i}, \frac{\bar{\alpha}}{k}\right) \cup B\left(\eta_{l}, \frac{\hat{\alpha}}{h}\right)\right)}|u|^{p-1} \bar{Z}_{0 i} \hat{Z}_{0 j} \\
= & : i_{1}+i_{2}+i_{3}+i_{4},
\end{aligned}
$$

Firstly, using the definition of $\bar{Z}_{0 i}$ and $\hat{Z}_{0 l}$,

$$
\begin{aligned}
i_{1} & \leqslant C \int_{B\left(\xi_{i}, \frac{\bar{\alpha}}{k}\right)}|u|^{p-1} \bar{Z}_{0 i} \frac{\lambda^{\frac{n-2}{2}}}{\left|y-\eta_{l}\right|^{n-2}} \leqslant C \mu^{\frac{n-2}{2}} \lambda^{\frac{n-2}{2}} \int_{B\left(0, \frac{\bar{\alpha}}{\mu k}\right)} U^{p-1} Z_{00} \\
& \leqslant C \mu^{\frac{n-2}{2}} \lambda^{\frac{n-2}{2}},
\end{aligned}
$$

where the second inequality follows by the change of variable $x=\xi_{i}+\mu y . i_{2}$ follows in the same way only by translating to $x=\eta_{l}+\lambda y$. On the other hand, we have that

$$
i_{3} \leqslant C \mu^{\frac{n-2}{2}} \lambda^{\frac{n-2}{2}} \int_{\mathbb{R}^{n} \backslash B(0,2)} \frac{1}{|y|^{4}|y|^{n-2}|y|^{n-2}} d y \leqslant C \mu^{\frac{n-2}{2}} \lambda^{\frac{n-2}{2}} .
$$

To estimate $i_{4}$ we take into account that, since $\xi_{i}$ and $\eta_{l}$ are separated, $\left|y-\xi_{i}\right|^{-(n-2)}$ and $\mid y-$ $\left.\eta_{l}\right|^{-(n-2)}$ cannot be singular at the same time, so the behavior of the integral comes determined by the singularity of only one of them. That is,

$$
\begin{aligned}
i_{4} & \leqslant C \mu^{\frac{n-2}{2}} \lambda^{\frac{n-2}{2}} \int_{B(0,2) \backslash\left(B\left(\xi_{i}, \frac{\bar{\alpha}}{k}\right) \cup B\left(\eta_{l}, \frac{\hat{\alpha}}{h}\right)\right)}|u|^{p-1} \frac{1}{\left|y-\xi_{i}\right|^{n-2}} \frac{1}{\left|y-\eta_{l}\right|^{n-2}} d y \\
& \leqslant C \mu^{\frac{n-2}{2}} \lambda^{\frac{n-2}{2}} \int_{B(0,2) \backslash\left(B\left(\xi_{i}, \frac{\bar{\alpha}}{k}\right) \cup B\left(\eta_{l}, \frac{\hat{\alpha}}{h}\right)\right)}\left(\frac{1}{\left|y-\xi_{i}\right|^{n-2}}+\frac{1}{\left|y-\eta_{l}\right|^{n-2}}\right) d y \\
& \leqslant C \mu^{\frac{n-2}{2}} \lambda^{\frac{n-2}{2}} .
\end{aligned}
$$

The case $\alpha, \beta \neq 0$ follows analogously just by noticing that

$$
\bar{Z}_{\alpha i} \sim \frac{\mu^{\frac{n-2}{2}}}{\left|y-\xi_{i}\right|^{n-1}}, \quad \hat{Z}_{\beta l} \sim \frac{\lambda^{\frac{n-2}{2}}}{\left|y-\eta_{l}\right|^{n-1}},
$$

and hence (2.3.5) is proved.

We now need the following result.

Proposition 2.3.1. The functions $\pi_{\alpha}$ can be decomposed as

$$
\pi_{\alpha}(y)=\sum_{j=1}^{k} \bar{\pi}_{\alpha j}(y)+\sum_{l=1}^{h} \hat{\pi}_{\alpha l}+\tilde{\pi}_{\alpha}(y),
$$

where

$$
\bar{\pi}_{\alpha j}(y)=\bar{\pi}_{\alpha 1}\left(e^{-\frac{2 \pi(j-1)}{k}} \bar{y}, \hat{y}, y^{\prime}\right), \quad \hat{\pi}_{\alpha l}(y)=\hat{\pi}_{\alpha 1}\left(\bar{y}, e^{-\frac{2 \pi(l-1)}{h}} \hat{y}, y^{\prime}\right)
$$


Furthermore, there exists a positive constant $C$ such that

$$
\begin{gathered}
\left\|\tilde{\pi}_{\alpha}\right\|_{*} \leqslant C\left(k^{1-\frac{n}{q}}+h^{1-\frac{n}{q}}\right), \quad \alpha=0,1, \ldots, n, \\
\left\|\overline{\bar{\pi}}_{\alpha 1}\right\|_{*} \leqslant C k^{-\frac{n}{q}}, \alpha=0, \ldots, n, \quad \text { where } \quad \overline{\bar{\pi}}_{\alpha 1}:=\mu^{\frac{n-2}{2}} \bar{\pi}_{\alpha 1}\left(\xi_{1}+\mu y\right), \\
\left\|\hat{\hat{\pi}}_{\alpha 1}\right\|_{*} \leqslant C h^{-\frac{n}{q}}, \alpha=0, \ldots, n, \quad \text { where } \quad \hat{\hat{\pi}}_{\alpha 1}:=\lambda^{\frac{n-2}{2}} \hat{\pi}_{\alpha 1}\left(\eta_{1}+\lambda y\right) .
\end{gathered}
$$

We omit the proof of this result.

Thanks to Proposition 2.3.1, we get

$$
\begin{gathered}
\left.\left|\int\right| u\right|^{p-1} Z_{\alpha 0} \pi_{\beta} \mid \leqslant C\left\|\tilde{\pi}_{\beta}\right\|_{*}, \\
\left.\left|\int\right| u\right|^{p-1} \bar{Z}_{\alpha i} \pi_{\beta}\left|\leqslant C\left\|\bar{\pi}_{\beta 1}\right\|_{*}, \quad\right| \int|u|^{p-1} \hat{Z}_{\alpha l} \pi_{\beta} \mid \leqslant C\left\|\hat{\pi}_{\beta 1}\right\|_{*} .
\end{gathered}
$$

Notice next (see (8.5) in [20] for a proof) that

$$
\int U^{p-1} Z_{00}^{2}=\int U^{p-1} Z_{10}^{2}>0
$$

and thus we can define

$$
t_{\beta}:=-\frac{1}{\int U^{p-1} Z_{00}^{2}} \int \varphi^{\perp}|u|^{p-1} \mathbf{z}_{\beta}
$$

which satisfies $\left|t_{\beta}\right| \leqslant C\left\|\varphi^{\perp}\right\|_{*}$, with $C$ independent of $k$ and $h$.

Consider (2.0.11) for $\beta=0$. Thus, by using the definition of $z_{0},(2.3 .1)-(2.3 .5),(2.3 .6),(2.3 .7)$ and Proposition 2.3.1 we get

$$
\begin{aligned}
& \sum_{\alpha=0}^{n}\left[c_{\alpha 0} \int_{\mathbb{R}^{n}} Z_{\alpha 0}|u|^{p-1} z_{0}+\sum_{j=1}^{k} \bar{c}_{\alpha j} \int_{\mathbb{R}^{n}} \bar{Z}_{\alpha j}|u|^{p-1} z_{0}+\sum_{l=1}^{h} \hat{c}_{\alpha l} \int_{\mathbb{R}^{n}} \hat{Z}_{\alpha l}|u|^{p-1} z_{0}\right] \\
& =c_{00} \int U^{p-1} Z_{00}^{2}-\sum_{j=1}^{k} \bar{c}_{0 j} \int U^{p-1} Z_{00}^{2}-\sum_{l=1}^{h} \hat{c}_{0 l} \int U^{p-1} Z_{00}^{2} \\
& -\sum_{j=1}^{k} \bar{c}_{1 j} \int U^{p-1} Z_{00}^{2}-\sum_{l=1}^{h} \hat{c}_{3 l} \int U^{p-1} Z_{00}^{2}+O\left(k^{1-\frac{n}{q}}+h^{1-\frac{n}{q}}\right) \mathcal{L}\left[\begin{array}{c}
c_{00} \\
\ldots \\
c_{n 0}
\end{array}\right] \\
& +O\left(k^{-\frac{n}{q}}\right) \overline{\mathcal{L}}\left[\begin{array}{c}
\bar{c}_{0} \\
\cdots \\
\bar{c}_{n}
\end{array}\right]+O\left(h^{-\frac{n}{q}}\right) \hat{\mathcal{L}}\left[\begin{array}{c}
\hat{c}_{0} \\
\cdots \\
\hat{c}_{n}
\end{array}\right],
\end{aligned}
$$

where $\mathcal{L}, \overline{\mathcal{L}}$ and $\hat{\mathcal{L}}$ are linear functions with coefficients uniformly bounded in $k$ and $h$. Identity (2.0.14) follows straightforward from here. (2.0.15)-(2.0.33) are obtained in the same way. 


\subsection{Proof of (2.0.10)}

We proceed as in [20, Section 9]. Indeed, we decompose $\varphi_{0}^{\perp}$ as

$$
\varphi_{0}^{\perp}=\sum_{\alpha=0}^{n} c_{\alpha 0} \varphi_{\alpha 0}^{\perp}, \quad \text { with } \quad L\left(\varphi_{\alpha 0}^{\perp}\right)=-L\left(Z_{\alpha 0}\right),
$$

which is equivalent to

$$
\Delta\left(\varphi_{\alpha 0}^{\perp}\right)+p \gamma U^{p-1}\left(\varphi_{\alpha 0}^{\perp}\right)+a_{0}(y) \varphi_{\alpha 0}^{\perp}=-L\left(Z_{\alpha 0}\right),
$$

where $a_{0}(y):=p \gamma\left(|u|^{p-1}-U^{p-1}\right)$. Adapting the arguments of 20] it can be seen that $a \in$ $L^{\frac{n}{2}}\left(\mathbb{R}^{n}\right)$,

$$
\begin{gathered}
|y|^{-n-2} L\left(Z_{00}\right)\left(|y|^{-2} y\right)=-L\left(Z_{00}\right)(y), \\
|y|^{-n-2} L\left(Z_{\alpha 0}\right)\left(|y|^{-2} y\right)=L\left(Z_{\alpha 0}\right)(y), \alpha=1, \ldots, n,
\end{gathered}
$$

and

$$
\left\|L\left(Z_{\alpha 0}\right)\right\|_{L^{\frac{2 n}{n+2}\left(\mathbb{R}^{n}\right)}} \leqslant C\left(\mu^{\frac{n-1}{n}}+\lambda^{\frac{n-1}{n}}\right) .
$$

We will solve (2.4.1) as a fixed point problem. Let us consider the problem

$$
L_{0}(\varphi)=h-a_{0}(y) \phi,
$$

where $L_{0}(\varphi):=\Delta \varphi+p \gamma U^{p-1} \varphi$ and $h \in L^{\frac{2 n}{n+2}}\left(\mathbb{R}^{n}\right)$ satisfies

$$
h(y)=|y|^{-n-2} h\left(|y|^{-2} y\right) .
$$

Let $T$ be the operator that associates to every $\phi$ the solution $\varphi$ to this problem, that is,

$$
\varphi=T\left(h-a_{0}(y) \phi\right) .
$$

Naming $A(\phi):=T\left(h-a_{0}(y) \phi\right)$ we are going to see that this operator is a contraction and that maps the ball

$$
B:=\left\{\phi \in \mathcal{D}^{1,2}\left(\mathbb{R}^{n}\right):\|\phi\|_{*} \leqslant C\left(\mu^{\frac{n-1}{n}}+\lambda^{\frac{n-1}{n}}\right), \phi(y)=|y|^{-n+2} \phi\left(|y|^{-2} y\right)\right\},
$$

into herself. Indeed, assume $\phi \in B$. Thus,

$$
a_{0}(y) \phi(y)=|y|^{-n-2} a_{0}\left(|y|^{-2} y\right) \phi\left(|y|^{-2} y\right),
$$

and, by [20, Proposition 9.1], we know that

$$
\|\varphi\|_{*} \leqslant C\left\|h-a_{0}(y) \phi\right\|_{L^{\frac{2 n}{n+2}}\left(\mathbb{R}^{n}\right)} \leqslant C\left(\|h\|_{L^{\frac{2 n}{n+2}\left(\mathbb{R}^{n}\right)}}+\left\|a_{0}(y) \phi\right\|_{L^{\frac{2 n}{n+2}\left(\mathbb{R}^{n}\right)}}\right),
$$

and $\varphi(y)=|y|^{2-n} \varphi\left(|y|^{-2} y\right)$.

We study the last term in two different regions. First, in

$$
\mathbb{R}^{n} \backslash\left(\left\{\cup_{j=1}^{k} B\left(\xi_{j}, \frac{\bar{\alpha}}{k}\right)\right\} \cup\left\{\cup_{l=1}^{h} B\left(\eta_{l}, \frac{\hat{\alpha}}{h}\right)\right\}\right)
$$

we can estimate $a_{0}$ as

$$
\left|a_{0}(y)\right| \leqslant C U^{p-2}\left[\sum_{j=1}^{k} \frac{\mu^{\frac{n-2}{2}}}{\left|y-\xi_{j}\right|^{n-2}}+\sum_{l=1}^{h} \frac{\lambda^{\frac{n-2}{2}}}{\left|y-\eta_{l}\right|^{n-2}}\right],
$$


and consequently,

$$
\int_{\mathbb{R}^{n} \backslash\left(\left\{\cup_{j=1}^{k} B\left(\xi_{j}, \frac{\bar{\alpha}}{k}\right)\right\} \cup\left\{\cup_{l=1}^{h} B\left(\eta_{l}, \frac{\hat{\alpha}}{h}\right)\right\}\right)}\left|a_{0}(y)\right|^{\frac{2 n}{n+2}} d y \leqslant C\left(k^{-(n-1)}+h^{-(n-1)}\right) .
$$

Consider now $j \in\{1, \ldots, k\}$ and the ball $B\left(\xi_{j}, \frac{\bar{\alpha}}{k}\right)$. Here

$$
\left|a_{0}(y)\right| \leqslant C\left|U_{\mu, \xi_{j}}(y)\right|^{p-1},
$$

and thus

$$
\begin{aligned}
\sum_{j=1}^{k} \int_{B\left(\xi_{j}, \frac{\bar{\alpha}}{k}\right)}\left|a_{0}(y)\right|^{\frac{2 n}{n+2}} d y & \leqslant C \sum_{j=1}^{k} \int_{B\left(\xi_{j}, \frac{\bar{\alpha}}{k}\right)}\left[\frac{\mu^{-\frac{n-2}{2}}}{1+\left|y-\xi_{j}\right|^{n-2}}\right]^{(p-1) \frac{2 n}{n+2}} \\
& \leqslant C k \mu^{-2 \frac{2 n}{n+2}} \mu^{n} \int_{B\left(0, \frac{1}{\mu k}\right)}\left[\frac{1}{1+|y|^{n-2}}\right]^{(p-1) \frac{2 n}{n+2}} d y \\
& \leqslant C k^{-(n-1)} .
\end{aligned}
$$

Likewise,

$$
\sum_{l=1}^{k} \int_{B\left(\eta_{l}, \frac{\hat{\alpha}}{h}\right)}\left|a_{0}(y)\right|^{\frac{2 n}{n+2}} d y \leqslant C h^{-(n-1)} .
$$

Putting (2.4.5), (2.4.6) and (2.4.7) together we conclude that if $\phi \in B$, then

$$
\left\|a_{0}(y) \phi\right\|_{L^{\frac{2 n}{n+2}\left(\mathbb{R}^{n}\right)}} \leqslant\|\phi\|_{L^{\infty}\left(\mathbb{R}^{n}\right)}\left\|a_{0}(y)\right\|_{L^{\frac{2 n}{n+2}\left(\mathbb{R}^{n}\right)}} \leqslant C\left(\mu^{\frac{n-1}{n}}+\lambda^{\frac{n-1}{n}}\right) .
$$

Furthermore

$$
\begin{aligned}
\left\|A\left(\phi_{1}\right)-A\left(\phi_{2}\right)\right\|_{*} & \leqslant C\left\|a_{0}(y)\left(\phi_{1}-\phi_{2}\right)\right\|_{L^{\frac{2 n}{n+2}}\left(\mathbb{R}^{n}\right)} \\
& \leqslant C\left\|a_{0}(y)\right\|_{L^{\frac{2 n}{n+2}}\left(\mathbb{R}^{n}\right)}\left\|\phi_{1}-\phi_{2}\right\|_{*} \\
& =o(1)\left\|\phi_{1}-\phi_{2}\right\|_{*},
\end{aligned}
$$

where $o(1)$ denotes a quantity which goes to zero when $k, h$ tend to infinity. Thus, $A$ defines a contraction mapping whenever

$$
\|h\|_{L^{\frac{2 n}{n+2}}} \leqslant C\left(\mu^{\frac{n-1}{n}}+\lambda^{\frac{n-1}{n}}\right) .
$$

Hence, considering $h=L\left(Z_{\alpha 0}\right)$, by (2.4.4) we conclude the existence of a solution to (2.4.1) satisfying

$$
\left\|\varphi_{\alpha 0}^{\perp}\right\|_{*} \leqslant C\left(\mu^{\frac{n-1}{n}}+\lambda^{\frac{n-1}{n}}\right) .
$$

Consider now $j \in\{1, \ldots, k\}, l \in\{1, \ldots, h\}$, and let us write,

$$
\begin{aligned}
& \bar{\varphi}_{j}^{\perp}=\sum_{\alpha=0}^{n} c_{\alpha j} \bar{\varphi}_{\alpha j}^{\perp}, \quad \text { with } \quad L\left(\bar{\varphi}_{\alpha j}^{\perp}\right)=-L\left(\bar{Z}_{\alpha j}\right), \\
& \hat{\varphi}_{l}^{\perp}=\sum_{\alpha=0}^{n} c_{\alpha l} \hat{\varphi}_{\alpha l}^{\perp}, \quad \text { with } \quad L\left(\hat{\varphi}_{\alpha l}^{\perp}\right)=-L\left(\hat{Z}_{\alpha l}\right) .
\end{aligned}
$$

Performing the change of variables

$$
\overline{\bar{\varphi}}_{j}^{\perp}(y):=\mu^{\frac{n-2}{2}} \bar{\varphi}_{\alpha j}^{\perp}\left(\mu y+\xi_{j}\right), \quad \hat{\hat{\varphi}}_{l}^{\perp}(y):=\lambda^{\frac{n-2}{2}} \hat{\varphi}_{\alpha l}^{\perp}\left(\lambda y+\eta_{l}\right),
$$


the previous equations turn into

$$
\begin{aligned}
& \Delta\left(\overline{\bar{\varphi}}_{j}^{\perp}\right)+p \gamma U^{p-1}\left(\overline{\bar{\varphi}}_{j}^{\perp}\right)+p \gamma \bar{a}_{j}(y) \overline{\bar{\varphi}}_{j}^{\perp}=\bar{h}_{j}(y), \\
& \Delta\left(\hat{\hat{\varphi}}_{l}^{\perp}\right)+p \gamma U^{p-1}\left(\hat{\hat{\varphi}}_{l}^{\perp}\right)+p \gamma \hat{a}_{l}(y) \hat{\hat{\varphi}}_{l}^{\perp}=\hat{h}_{l}(y),
\end{aligned}
$$

where

$$
\begin{gathered}
\bar{a}_{j}(y):=p \gamma\left[\left(\mu^{-\frac{n-2}{2}}|u|\left(\mu y+\xi_{j}\right)\right)^{p-1}-U^{p-1}\right], \quad \bar{h}_{j}(y):=-\mu^{\frac{n+2}{2}} L\left(\bar{Z}_{\alpha j}\right)\left(\mu y+\xi_{j}\right), \\
\hat{a}_{l}(y):=p \gamma\left[\left(\lambda^{-\frac{n-2}{2}}|u|\left(\lambda y+\eta_{l}\right)\right)^{p-1}-U^{p-1}\right], \quad \hat{h}_{l}(y):=-\lambda^{\frac{n+2}{2}} L\left(\hat{Z}_{\alpha l}\right)\left(\lambda y+\eta_{l}\right) .
\end{gathered}
$$

Performing an analogous fixed point argument we conclude (2.0.10).

\subsection{Final ARgument}

Let $\left[\begin{array}{c}c_{0} \\ c_{1} \\ \cdots \\ c_{n}\end{array}\right]$ be the solution to (2.0.36) provided by Proposition 2.0.3, and let $t_{0}, t_{1}, t_{2}, t_{3}, t_{4}$,

$\bar{t}_{0}, \bar{t}_{1}, \bar{t}_{2}, \hat{t}_{0}, \hat{t}_{1}, \hat{t}_{2}$, and $t_{\alpha}, \bar{\nu}_{\alpha 1}, \bar{\nu}_{\alpha 2}, \hat{\nu}_{\alpha 1}, \hat{\nu}_{\alpha 2}, \alpha=5, \ldots, n$, be the associated parameters. Thus, it follows straightforward the existence of a unique vector of parameters

$$
\begin{gathered}
\left(t_{0}^{*}, \ldots, t_{4}^{*}, \bar{t}_{0}^{*}, \bar{t}_{1}^{*}, \bar{t}_{2}^{*}, \hat{t}_{0}^{*}, \hat{t}_{1}^{*}, \hat{t}_{2}^{*}, t_{5}^{*}, \bar{\nu}_{51}^{*}, \bar{\nu}_{52}^{*}, \hat{\nu}_{51}^{*}, \hat{\nu}_{52}^{*}, \ldots, t_{n}^{*}, \bar{\nu}_{n 1}^{*}, \bar{\nu}_{n 2}^{*}, \hat{\nu}_{n 1}^{*}, \hat{\nu}_{n 2}^{*}\right) \\
\text { such that }\left[\begin{array}{c}
c_{0} \\
c_{1} \\
\ldots \\
c_{n}
\end{array}\right] \text { solves the system in Proposition 2.0.2 and, equivalently, (2.0.11). Moreover, } \\
\left\|\left(t_{0}^{*}, t_{1}^{*}, t_{2}^{*}, t_{3}^{*}, t_{4}^{*}, \bar{t}_{0}^{*}, \bar{t}_{1}^{*}, \bar{t}_{2}^{*}, \hat{t}_{0}^{*}, \hat{t}_{1}^{*}, \hat{t}_{2}^{*}, t_{5}^{*}, \bar{\nu}_{51}^{*}, \bar{\nu}_{52}^{*}, \hat{\nu}_{51}^{*}, \hat{\nu}_{52}^{*}, \ldots, t_{n}^{*}, \bar{\nu}_{n 1}^{*}, \bar{\nu}_{n 2}^{*}, \hat{\nu}_{n 1}^{*}, \hat{\nu}_{n 2}^{*}\right)\right\| \leqslant C\left\|\varphi^{\perp}\right\|,
\end{gathered}
$$

and therefore

$$
\left\|\left[\begin{array}{c}
c_{0} \\
c_{1} \\
\cdots \\
c_{n}
\end{array}\right]\right\| \leqslant C\left\|\varphi^{\perp}\right\|
$$

This estimate, together with (2.0.10), allows us to conclude

$$
c_{\alpha}=0 \quad \forall \alpha=0, \ldots, n,
$$

and thus $\varphi^{\perp} \equiv 0$. Replacing this in (2.0.8) the proof of Theorem 2.0.1 is complete.

\subsection{APPENDIX}

According to their definitions, see (0.0.12) $-(0.0 .17)$ and (2.0.2), it is convenient to rewrite the functions $z_{\alpha}$ as

$$
z_{0}(y)=Z_{00}(y)-\sum_{j=1}^{k}\left[\bar{Z}_{0 j}(y)+\bar{Z}_{1 j}(y)\right]-\sum_{l=1}^{h}\left[\hat{Z}_{0 l}(y)+\hat{Z}_{3 l}(y)\right],
$$




$$
\begin{aligned}
& z_{1}(y)=Z_{10}(y)-\sum_{j=1}^{k} \frac{\cos \bar{\theta}_{j} \bar{Z}_{1 j}(y)-\sin \bar{\theta}_{j} \bar{Z}_{2 j}(y)}{\sqrt{1-\mu^{2}}}-\sum_{l=1}^{h} \hat{Z}_{1 l}(y), \\
& z_{2}(y)=Z_{20}(y)-\sum_{j=1}^{k} \frac{\sin \bar{\theta}_{j} \bar{Z}_{1 j}(y)+\cos \bar{\theta}_{j} \bar{Z}_{2 j}(y)}{\sqrt{1-\mu^{2}}}-\sum_{l=1}^{h} \hat{Z}_{2 l}(y), \\
& z_{3}(y)=Z_{30}(y)-\sum_{j=1}^{k} \bar{Z}_{3 j}(y)-\sum_{l=1}^{h} \frac{\cos \hat{\theta}_{l} \hat{Z}_{3 l}(y)-\sin \hat{\theta}_{l} \hat{Z}_{4 l}(y)}{\sqrt{1-\lambda^{2}}}, \\
& z_{4}(y)=Z_{40}(y)-\sum_{j=1}^{k} \bar{Z}_{4 j}(y)-\sum_{l=1}^{h} \frac{\sin \hat{\theta}_{l} \hat{Z}_{3 l}(y)+\cos \hat{\theta}_{l} \hat{Z}_{4 l}(y)}{\sqrt{1-\lambda^{2}}}, \\
& z_{\alpha}(y)=Z_{\alpha 0}(y)-\sum_{j=1}^{k} \bar{Z}_{\alpha j}(y)-\sum_{l=1}^{h} \hat{Z}_{\alpha l}(y), \quad \alpha=5, \ldots, n \\
& z_{n+1}(y)=-\sum_{j=1}^{k} \bar{Z}_{2 j}(y), \quad z_{n+2}(y)=-\sum_{l=1}^{h} \hat{Z}_{4 l}(y) \\
& z_{n+7}(y)=-\sqrt{1-\mu^{2}} \sum_{j=1}^{k} \cos \bar{\theta}_{j} \bar{Z}_{3 j}(y)+\sqrt{1-\lambda^{2}} \sum_{l=1}^{h} \cos \hat{\theta}_{l} \hat{Z}_{1 l}(y), \\
& z_{n+8}(y)=-\sqrt{1-\mu^{2}} \sum_{j=1}^{k} \cos \bar{\theta}_{j} \bar{Z}_{4 j}(y)+\sqrt{1-\lambda^{2}} \sum_{l=1}^{h} \sin \hat{\theta}_{l} \hat{Z}_{1 l}(y), \\
& z_{n+\alpha+4}(y)=-\sqrt{1-\mu^{2}} \sum_{j=1}^{k} \cos \bar{\theta}_{j} \bar{Z}_{\alpha j}(y), \quad \alpha=5, \ldots, n, \\
& z_{2 n+5}(y)=-\sqrt{1-\mu^{2}} \sum_{j=1}^{k} \sin \bar{\theta}_{j} \bar{Z}_{3 j}+\sqrt{1-\lambda^{2}} \sum_{l=1}^{h} \cos \hat{\theta}_{l} \hat{Z}_{2 l} \\
& z_{2 n+6}(y)=-\sqrt{1-\mu^{2}} \sum_{j=1}^{k} \sin \bar{\theta}_{j} \bar{Z}_{4 j}+\sqrt{1-\lambda^{2}} \sum_{l=1}^{h} \sin \hat{\theta}_{l} \hat{Z}_{2 l}
\end{aligned}
$$

and, for $\alpha=5, \ldots, n$,

$$
\begin{gathered}
z_{2 n+\alpha+2}(y)=-\sqrt{1-\mu^{2}} \sum_{j=1}^{k} \sin \bar{\theta}_{j} \bar{Z}_{\alpha j}, \quad z_{3 n+\alpha-2}(y)=-\sqrt{1-\lambda^{2}} \sum_{l=1}^{h} \sin \hat{\theta}_{l} \hat{Z}_{3 l}, \\
z_{4 n+\alpha-6}(y)=-\sqrt{1-\lambda^{2}} \sum_{l=1}^{h} \cos \hat{\theta}_{l} \hat{Z}_{4 l} .
\end{gathered}
$$

The proof of the above identities follows from straightforward computations, and the symmetry properties for $U(y)+\psi(y)$, for $U_{\mu, \xi_{j}}(y)+\bar{\phi}_{j}(y)$ and $U_{\lambda, \eta_{l}}(y)+\hat{\phi}_{l}(y)$ respectively. 
A less straightforward computation gives that, $\alpha=n+3, n+4, n+5, n+6$, we have

$$
\begin{aligned}
& \mathbf{z}_{n+3}(y)=z_{1}-2 \sqrt{1-\mu^{2}} \sum_{j=1}^{k} \cos \bar{\theta}_{j}\left[\bar{Z}_{0 j}(y)+\bar{Z}_{1 j}\right], \\
& \mathbf{z}_{n+4}(y)=z_{2}-2 \sqrt{1-\mu^{2}} \sum_{j=1}^{k} \sin \bar{\theta}_{j}\left[\bar{Z}_{0 j}(y)+\bar{Z}_{1 j}\right], \\
& \mathbf{z}_{n+5}(y)=z_{1}-2 \sqrt{1-\lambda^{2}} \sum_{l=1}^{h} \cos \hat{\theta}_{l}\left[\hat{Z}_{0 l}(y)+\hat{Z}_{3 l}\right], \\
& \mathbf{z}_{n+6}(y)=z_{1}-2 \sqrt{1-\lambda^{2}} \sum_{j=1}^{h} \sin \hat{\theta}_{l}\left[\hat{Z}_{0 l}(y)+\hat{Z}_{4 l}\right] .
\end{aligned}
$$

We shall prove the validity of the first identity in (2.6.1). The proofs of the validity of the the other expressions in (2.6.1) are similar. We write

$$
\mathbf{z}_{n+3}=z_{1}+T(u), \quad T(u):=\left(|y|^{2}-1\right) \frac{\partial u}{\partial y_{1}}-2 y_{1}\left(\frac{n-2}{2} u(y)+\nabla u(y) \cdot y\right) .
$$

Thus (2.6.1) follows from (2.0.2) and from

$$
T(u)=-2 \sum_{j=1}^{k} \xi_{j 1}\left[\bar{Z}_{0 j}+\nabla\left(U_{\mu, \xi_{j}}+\bar{\phi}_{j}\right)(y) \cdot \xi_{j}\right] .
$$

From the explicit expression of $u$ in (2.0.5), we get

$$
T(u)=T(U+\psi)-\sum_{j=1}^{k} T\left(U_{\mu, \xi_{j}}+\bar{\phi}_{j}\right)-\sum_{l=1}^{h} T\left(U_{\lambda, \eta_{l}}+\hat{\phi}_{l}\right) .
$$

We shall first show that $T(U+\psi)(y) \equiv 0$, and $T\left(U_{\lambda, \eta_{l}}+\hat{\phi}_{l}\right)(y) \equiv 0$ for any $l=1, \ldots, h$.

Observe that, if $v$ is any smooth function and if we define $h(z):=\frac{\partial}{\partial z_{1}}\left(|z|^{2-n} v\left(\frac{z}{|z|^{2}}\right)\right)$, then we have

$$
\begin{aligned}
h(z) & =-\frac{2 z_{1}}{|z|^{n}}\left[\frac{n-2}{2} v\left(\frac{z}{|z|^{2}}\right)+\nabla v\left(\frac{z}{|z|^{2}}\right) \cdot\left(\frac{z}{|z|^{2}}\right)\right] \\
& +\frac{1}{|z|^{n}} \frac{\partial v}{\partial z_{1}}\left(\frac{z}{|z|^{2}}\right),
\end{aligned}
$$

and $g(y):=\frac{1}{|y|^{n-2}} h\left(\frac{y}{|y|^{2}}\right)$ takes the form

$$
g(y)=-2 y_{1}\left[\frac{n-2}{2} v(y)+\nabla v(y) \cdot y\right]+|y|^{2} \frac{\partial v}{\partial y_{1}}(y) .
$$

With this is mind, one gets that if $v$ is Kelvin invariant $v(y)=|y|^{n-2} v\left(\frac{y}{|y|^{2}}\right)$, then

$$
\frac{1}{|y|^{n-2}} \frac{\partial v}{\partial y_{1}}\left(\frac{y}{|y|^{2}}\right)=-2 y_{1}\left[\frac{n-2}{2} v(y)+\nabla v(y) \cdot y\right]+|y|^{2} \frac{\partial v}{\partial y_{1}}(y)
$$


On the other hand, if $v$ is Kelvin invariant (with respect to the origin) and even in $y_{1}$, then also the function $\frac{\partial v}{\partial y_{1}}$ is Kelvin invariant, that is $\frac{\partial v}{\partial y_{1}}(y)=|y|^{n-2} \frac{\partial v}{\partial y_{1}}\left(\frac{y}{|y|^{2}}\right)$. By (2.6.3) , we get that any function $v$ which is invariant under Kelvin transform (with respect to the origin) and even in the $y_{1}$ direction, one that $T(v)(y) \equiv 0$. Since the functions $(U+\psi)(y)$, and $\left(U_{\lambda, \eta_{l}}+\hat{\phi}_{l}\right)(y)$ for any $l=1, \ldots, h$ are invariant under Kelvin transform and even in $y_{1}$, we get the proof of our claim.

Let us fix $j \in\{1, \ldots, k\}$. We write, for $v(y)=\left(U_{\mu, \xi_{j}}+\bar{\phi}_{j}\right)(y)$,

$$
\begin{aligned}
T\left(U_{\mu, \xi_{j}}+\bar{\phi}_{j}\right)(y) & =\underbrace{\left(|y|^{2}-1\right) \frac{\partial v}{\partial y_{1}}(y)-2\left(y-\xi_{j}\right)_{1}\left[\frac{n-2}{2} v(y)+\nabla v(y) \cdot y\right]}_{=: T_{j}(v)} \\
& -2\left(\xi_{j}\right)_{1}\left[\bar{Z}_{0 j}(y)+\nabla v(y) \cdot \xi_{j}\right] .
\end{aligned}
$$

We claim that $T_{j}\left(U_{\mu, \xi_{j}}+\bar{\phi}_{j}\right)(y) \equiv 0$. To prove this fact, we recall that

$$
v(y):=\left(U_{\mu, \xi_{j}}+\bar{\phi}_{j}\right)(y)=\mu^{-\frac{n-2}{2}}\left(U+\overline{\bar{\phi}}_{1}\right)\left(\frac{y-\xi_{j}}{\mu}\right),
$$

see Section 1.2. Also, $\mu$ and $|\xi|$ are related so that $U_{\mu, \xi_{j}}+\bar{\phi}_{j}$ is invariant under Kelvin transform. Thus, from (2.6.3), we get

$$
T_{j}(v)(y)=\frac{1}{|y|^{n-2}} \frac{\partial v}{\partial y_{1}}\left(\frac{y}{|y|^{2}}\right)-\frac{\partial v}{\partial y_{1}}(y)+2\left(\xi_{j}\right)_{1}\left[\frac{n-2}{2} v(y)+\nabla v(y) \cdot y\right] .
$$

We note that, in this case, $U_{\mu, \xi_{j}}+\bar{\phi}_{j}$ is not even in the $y_{1}$ variable, so that one gets

$$
\frac{1}{|y|^{n-2}} \frac{\partial v}{\partial y_{1}}\left(\frac{y}{|y|^{2}}\right)=\frac{\partial v}{\partial y_{1}}(y)-\left(\xi_{j}\right)_{1}[(n-2) v(y)+2 \nabla v(y) \cdot y]
$$

This concludes the proof of (2.6.2).

\section{REFERENCES}

[1] C.Brenier, N. Kapouleas, Complete Constant Mean Curvature Hypersurfaces in Euclidean space of dimension four or higher, arXiv:1707.04008.

[2] L.A. Caffarelli, B. Gidas, J. Spruck, Asymptotic symmetry and local behavior of semilinear elliptic equations with critical Sobolev growth, Comm. Pure Appl. Math. 42 (1989), 271-297.

[3] J. Dávila, M. del Pino, Y. Sire, Nondegeneracy of the bubble in the critical case for nonlocal equations, Proc. Amer. Math. Soc., 141(11) (2013), 3865-3870.

[4] M. del Pino, M. Musso, F. Pacard, A. Pistoia, Large energy entire solutions for the Yamabe equation. Journal of Differential Equations 251 (2011), 2568-2597.

[5] M. del Pino, M. Musso, F. Pacard. A. Pistoia. Torus action on $S^{n}$ and sign changing solutions for conformally invariant equations. Annali della Scuola Normale Superiore di Pisa (5) 12 (2013), no. 1, 209-237.

[6] W. Ding, On a conformally invariant elliptic equation on $R^{n}$, Communications in Mathematical Physics 107 (1986), 331-335.

[7] T. Duyckaerts, C. Kenig, F. Merle, Solutions of the focusing nonradial critical wave equation with the compactness property, to appear in Ann. Sc. Norm. Super. Pisa Cl. Sci.

[8] T. Duyckaerts, C. Kenig, F. Merle, Profiles of bounded radial solutions of the focusing, energy-critical wave equation. Geom. Funct. Anal. 22 (2012), no. 3, 639-698. 
[9] T. Duyckaerts, C. Kenig, F. Merle, Universality of the blow-up profile for small type II blow-up solutions of the energy-critical wave equation: the nonradial case. J. Eur. Math. Soc. (JEMS) 14 (2012), no. 5, $1389-1454$.

[10] R. L. Frank, E. Lenzmann, Uniqueness and nondegeneracy of ground states for $(-\Delta)^{s} Q+Q-Q^{\alpha+1}=0$ in R. Acta Math. 210 (2013), no. 2, 26117318.

[11] R. L. Frank, E. Lenzmann, L. Silvestre, Uniqueness of radial solutions for the fractional Laplacian. Comm. Pure Appl. Math. 69 (2016), no. 9, 1671-1726.

[12] E. Hebey, Introduction à l'analyse non linéaire sur les variétées, Diderot éditeur (1997).

[13] E. Hebey, M. Vaugon, Existence and multiplicity of nodal solutions for nonlinear elliptic equations with critical Sobolev growth.J. Funct. Anal. 119 (1994), no. 2, 298-318.

[14] N. Kapouleas, Minimal surfaces in the round three-sphere by doubling the equatorial two-sphere, I, J. Diff. Geom. (2015).

[15] C. Kenig, F.Merle, Global well-posedness, scattering and blow-up for the energy-critical focusing non-linear wave equation in the radial case, Invent. Math. 166 (2006), 645-675.

[16] C. Kenig, F. Merle, Global well-posedness, scattering and blow-up for the energy-critical focusing nonlinear wave equation, Acta Math. 201 (2008), 147-212.

[17] I. Kra, R. Simanca, On circulant matrices, Notices. Amer. Math. Soc. 59, no. 3, (2012), 368-377.

[18] J. Krieger, W. Schlag, D. Tataru, Slow blow-up solutions for the $H^{1}\left(\mathbb{R}^{3}\right)$ critical focusing semilinear wave equation. Duke Math. J. 147 (2009), 1-53.

[19] M. K. Kwong, Uniqueness of positive solutions of $\Delta u-u+u^{p}=0$ in $R^{n}$, Arch. Rational Mech. Anal. 105 (1989), pp. 243-266.

[20] M. Musso, J. Wei, Nondegeneracy of nodal solutions to the critical Yamabe problem. Communications in Mathematical Physics, Volume 340, Issue 3, (2015), 1049-1107.

[21] O. Rey, The role of the Green's function in a nonlinear elliptic equation involving the critical Sobolev exponent. J. Funct. Anal. 89 (1990), no. 1, 1-52.

[22] F. Robert, J. Vetois, Examples of non-isolated blow-up for perturbations of the scalar curvature equation on non locally conformally flat manifolds, to appear in J. of Differential Geometry.

[23] F. Robert, J. Vetois, Sign-changing solutions to elliptic second order equations: glueing a peak to a degenerate critical manifold, Calc. Var. Partial Differential Equations 54 (2015), no. 1, 693-716.

(Maria Medina) Facultad de Matemáticas, Pontificia Universidad Católica de Chile, Avenida Vicuña Mackenna 4860, Santiago, Chile

E-mail address: mamedinad@mat.puc.cl

(Monica Musso) Facultad de Matemáticas, Pontificia Universidad Católica de Chile, Avenida Vicuña Mackenna 4860, Santiago, Chile

E-mail address: musso@mat.puc.cl $1 \mathrm{Z} 2$

(Juncheng Wei) Department of Mathematics, University of British Columbia, Vancouver, BC V6T

E-mail address: jcwei@math.ubc.ca 